\title{
A Study of Hadronic Calibration Schemes for Pion Test Beam Data in the ATLAS Forward Calorimeter
}

\author{
by
}

\author{
Thomas G. McCarthy \\ A thesis submitted to the \\ Faculty of Graduate Studies and Research \\ in partial fulfillment of the requirements \\ for the degree of \\ Master of Science \\ Ottawa-Carleton Institute for Physics \\ Department of Physics \\ Carleton University \\ Ottawa, Ontario, Canada \\ June, 2010 \\ (C) copyright \\ Thomas G. McCarthy, 2010
}


Library and Archives Canada

Published Heritage

Branch

395 Wellington Street Ottawa ON K1A ON4 Canada
Bibliothèque et

Archives Canada

Direction du

Patrimoine de l'édition

395 , rue Wellington

Ottawa ON K1A ON4

Canada
Your file Votre référence
ISBN: 978-0-494-71589-5
Our file Notre référence
ISBN: 978-0-494-71589-5

\section{NOTICE:}

The author has granted a nonexclusive license allowing Library and Archives Canada to reproduce, publish, archive, preserve, conserve, communicate to the public by telecommunication or on the Internet, loan, distribute and sell theses worldwide, for commercial or noncommercial purposes, in microform, paper, electronic and/or any other formats.

The author retains copyright ownership and moral rights in this thesis. Neither the thesis nor substantial extracts from it may be printed or otherwise reproduced without the author's permission.
AVIS:

L'auteur a accordé une licence non exclusive permettant à la Bibliothèque et Archives Canada de reproduire, publier, archiver, sauvegarder, conserver, transmettre au public par télécommunication ou par l'Internet, prêter, distribuer et vendre des thèses partout dans le monde, à des fins commerciales ou autres, sur support microforme, papier, électronique et/ou autres formats.

L'auteur conserve la propriété du droit d'auteur et des droits moraux qui protège cette thèse. Ni la thèse ni des extraits substantiels de celle-ci ne doivent être imprimés ou autrement reproduits sans son autorisation.
In compliance with the Canadian Privacy Act some supporting forms may have been removed from this thesis.

While these forms may be included in the document page count, their removal does not represent any loss of content from the thesis.
Conformément à la loi canadienne sur la protection de la vie privée, quelques formulaires secondaires ont été enlevés de cette thèse.

Bien que ces formulaires aient inclus dans la pagination, il n'y aura aucun contenu manquant.

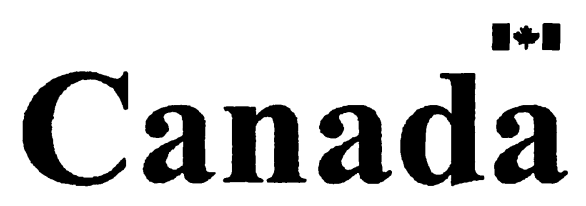




\section{Abstract}

The ATLAS forward calorimeters constitute a small though important fraction of the detector's calorimeter system, designed in part to accurately and precisely measure the energy of particles and jets of particles originating from the collisions of high-energy protons at the detector's centre. The application of hadronic weights, a practice common in high-energy calorimetry, provides a means of compensation for the fraction of energy which is deposited by particles in the detector, but which is invisible to the detector due to the nature of hadronic showers.

Explored here are various schemes of extracting hadronic weights, as well as the application of such weights, based on pion data from the 2003 ATLAS forward calorimeter test beam. During the collection of test beam data, beams of both pions and electrons of known energy, ranging from 10 to $200 \mathrm{GeV}$, were fired at specific points of an isolated detector in order to understand its response. The improvement in noise-subtracted energy resolution with respect to the electromagnetic scale results, based on a weighting scheme which takes advantage of the transverse properties of hadronic showers, is shown to be $34.5 \%$ at an energy of $200 \mathrm{GeV}$. In terms of the energy response, it is shown that upon application of hadronic weights for such a weighting scheme, one is able to reconstruct the pion energy to within $5 \%$ of its true value for all test beam energies above $40 \mathrm{GeV}$.

It is also shown that hadronic weights can be applied without taking advantage of the tracking information used in the test beam setup, in an effort to show the feasibility of such methods in the analysis of ATLAS collision data. Furthermore, the difficulties present in accurately reconstructing the energy in the high- $\eta$ region of the detector due to the presence of the ATLAS beam pipe and the means of compensating for such difficulties are investigated. 


\section{Acknowledgements}

I would like to thank the various members of the Carleton University ATLAS group for their ongoing support throughout my M.Sc., as well as the members of the FCal test beam group from the University of Toronto.

In particular, I am greatly indebted to Louise Heelan, a fellow graduate student at Carleton and member of the FCal test beam group, for innumerable conversations, offers of tips and suggestions, and in general for being there as someone off of whom to bounce ideas in return for invaluable feedback. In addition, Dag Gillberg, a postdoctoral fellow within the Carleton ATLAS group, has been a constant source of support throughout the second year of my program. An avuncular role model, Dag has always been eager to teach me the 'tips of the trade', and is consistently inquiring, with sincere interest, as to my latest progress - the latter providing me with more motivation and impetus than he might realize. I wish to thank both Louise and Dag for getting me up to speed with the details of the FCal test beam framework in order to spend as much time as possible moving forward with the analysis for the sake of the group.

Many thanks to Prof. Gerald Oakham for his patience, his trust, and his steady support throughout my program.

I would also like to acknowledge the generous support of the Natural Sciences and Engineering Research Council of Canada.

\section{(1) Mssece}




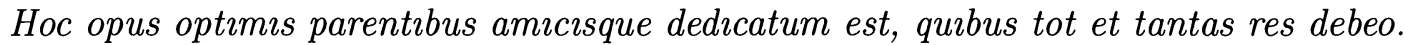




\section{Contents}

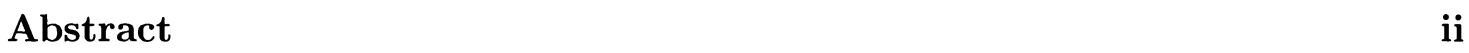

Acknowledgments $\quad$ iii

Dedication $\quad$ iv

List of Tables $\quad$ ix

List of Figures $\quad$ x

Glossary of Terms $\quad$ xiv

1 Introduction and Motivation $\quad 1$

1.1 General Introduction . . . . . . . . . . . . . . . . . . . . . . . . . 1

1.2 The Standard Model of Particle Physics . . . . . . . . . . . . . . . 2

1.3 The Large Hadron Collider at CERN . . . . . . . . . . . . . . . . . . 5

1.4 The ATLAS Experiment . . . . . . . . . . . . . . . 7

1.4.1 The ATLAS Coordinate System . . . . . . . . . . . . . . 11

1.5 Motivation for the Present Analysis . . . . . . . . . . . . . . . . 12

2 Calorimetry in Collider Experiments $\quad 14$

2.1 Introduction . . . . . . . . . . . . . . . . . 14

2.2 Sampling Calorimeters . . . . . . . . . . . . . . . 15

2.3 Interactions of Particles within the Calorimeter $\ldots \ldots \ldots \ldots$ 
2.4 Properties of Electromagnetic Showers . . . . . . . . . . . . . . 21

2.5 Properties of Hadronic Showers . . . . . . . . . . . . . . . . 24

2.6 Energy Response for Electrons . . . . . . . . . . . . . . . . . . 27

2.7 Energy Response for Hadrons _. . . . . . . . . . . . . . . . . 28

3 The ATLAS Forward Calorimeters 30

3.1 The ATLAS Liquid Argon Calorimeter System . . . . . . . . . . . . . . . 30

3.2 The ATLAS Forward Calorimeters . . . . . . . . . . . . . . . 31

3.2.1 Design and Construction . . . . . . . . . . . . . . 32

3.2.2 Commissioning and Ongoing Work . . . . . . . . . . . 37

42003 FCal Test Beam $\quad 38$

4.1 Test Beam Setup and Overview . . . . . . . . . . . . . . 38

4.2 Instrumentation During Data Collection . . . . . . . . . . . . . . 39

4.2.1 BPC Tracking Information and Beam Envelope Cuts . . . . . . . 40

4.2.2 Tail Catcher, Muon Counter and Beam Stop . . . . . . . . . . . 41

4.2.3 Čerenkov Detector and CEDAR Cuts . . . . . . . . . . . . . 42

4.2.4 Summary of Standard Data Cuts . . . . . . . . . . . . . . . 43

4.3 Beam Impact Points . . . . . . . . . . . . . . . . . . . . . 43

4.3.1 Modelling of Upstream Material . . . . . . . . . . . . . . . 46

4.4 Summary of Pion and Electron Data . . . . . . . . . . . . . . . . 47

4.5 Noise Treatment . . . . . . . . . . . . . . . . . . . . . . . 48

4.6 Offline Test Beam Data Analysis . . . . . . . . . . . . . . . . 49

5 Reconstructing Energy in the Forward Calorimeter 50

5.1 Summing Energy in the FCal Modules . . . . . . . . . . . . . . 51

5.1 .1 Inclusion of Active Readout Channels . . . . . . . . . . . . 51

5.1 .2 Cell Clustering Algorithms . . . . . . . . . . . . . 52

5.1.3 Double Gaussian Fit for the Reconstructed Energy . . . . . . . . 57

5.2 Energy resolution . . . . . . . . . . . . . . . . . 59

5.2.1 Noise-Subtracted Energy Resolution . . . . . . . . . . . 60 
5.3 Summary of Electron Data Results . . . . . . . . . . . . . 61

5.4 Summary of Introductory Sections . . . . . . . . . . . . . . 63

6 Pion Data Analysis Using Modular Hadronic Weights 64

6.1 Modular Weights as a Simple Weighting Scheme . . . . . . . . . . . . 64

6.2 Modular Weight Derivation _. . . . . . . . . . . . . 66

6.3 Errors on Extracted Weights . . . . . . . . . . . . . . . . . 72

6.4 Modular Weight Values for Point $4 \mathrm{~L} \ldots \ldots \ldots \ldots \ldots$

6.4.1 Modular Weights Using Cylindrical Clustering . . . . . . . . . . 74

6.4.2 Modular Weights Using Topological Clustering . . . . . . . . . 76

6.5 Modular Weights For Points 1, 2, 3 and $4 \mathrm{H} \ldots \ldots \ldots \ldots \ldots$

7 Modular Hadronic Weight Results $\quad 78$

7.1 Choice of Energy-Independent Weights . . . . . . . . . . . . 78

7.2 Reconstructed Energy _. . . . . . . . . . . . . . . . . 80

7.2.1 Results Using Cylindrical Clustering . . . . . . . . . . . . 80

7.2 .2 Results Using Topological Clustering . . . . . . . . . . . . . 83

7.3 Noise-Subtracted Energy Resolution . . . . . . . . . . . . . . . . . 84

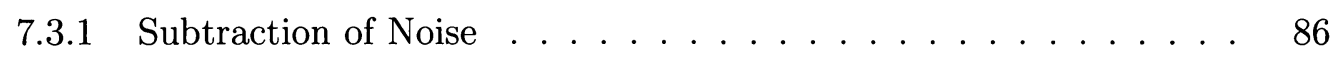

7.3.2 Comparison of Noise-Subtracted Energy Resolution . . . . . . 88

7.4 Extension to Alternative Weighting Schemes . . . . . . . . . . . . 89

8 Pion Data Analysis Using Radial Hadronic Weights 91

8.1 Radial Weighting Scheme As a Next Approach . . . . . . . . . . . . 91

8.2 A Look at Radial Profiles . . . . . . . . . . . . . . . . . . . . 94

8.2 .1 Number of Included Readout Channels . . . . . . . . . . . . 94

8.2 .2 Energy Density . . . . . . . . . . . . . . . . . . 98

8.3 Deriving Radial Weights . . . . . . . . . . . . . . . . . . . 101

8.3.1 Radial Weights: Standard Setup . . . . . . . . . . . . . . . . 101

8.3.2 Radial Weights: Simplified Setup . . . . . . . . . . . . . 106

8.3.3 Radial Weights: Simplified Setup Without Tracking . . . . . . . 109 
8.4 Summary of Radial Weights . . . . . . . . . . . . . . . . . 116

9 Radial Hadronic Weight Results $\quad 117$

9.1 Application of Derived Radial Weights . . . . . . . . . . . . . 117

9.2 Reconstructed Energy . . . . . . . . . . . . . . . 117

9.2.1 Cylindrical Clustering Reconstructed Energy Results . . . . . . . 117

9.2.2 Topological Clustering Reconstructed Energy Results . . . . . . . 119

9.3 Noise-Subtracted Energy Resolution . . . . . . . . . . . . . . . 120

9.3.1 Cylindrical Clustering Resolution Results . . . . . . . . . . . . . . 120

9.3.2 Topological Clustering Resolution Results . . . . . . . . . . . . . 122

9.4 Overall Summary of Weighting Results . . . . . . . . . . . . . . . 125

10 Inner Edge Energy Reconstruction $\quad 128$

10.1 Energy Loss at the Inner Edge of the FCal . . . . . . . . . . . . . . 128

10.2 Reconstructed Energy Radial Profile . . . . . . . . . . . . . . . . 129

10.2.1 Energy Profile at the Electromagnetic Scale . . . . . . . . . . . 129

10.2.2 Energy Profile with Radial Weight Application . . . . . . . . . . . 132

10.3 Summary of Energy Profiles at the Inner Edge . . . . . . . . . . . 133

11 Conclusion $\quad 135$

$\begin{array}{ll}\text { A Additional Plots } & 139\end{array}$

A.1 4 H Modular Weight Values . . . . . . . . . . . . . . . . . . . . . 139

A.2 Modular Weight Results . . . . . . . . . . . . . . . . . . . . . . 141

A.3 Radial Weight Values . . . . . . . . . . . . . . . . . . . 144

A.4 Reconstructed Energy Profiles . . . . . . . . . . . . . . . 150

B Radial Profiles of Included Cells $\quad 151$

$\begin{array}{ll}\text { References } & 153\end{array}$ 


\section{List of Tables}

2.1 Summary of nuclear and atomic properties for copper and tungsten . . . 26

2.2 Typical shower depths for particles in a copper or tungsten volume . . . 27

3.1 Design values of interest for the FCal modules . . . . . . . . . . . . . . 34

4.1 Pseudorapidity $\eta$ and polar angle $\theta$ values for selected beam points . . 45

4.2 Summary of pion data for outer points . . . . . . . . . . . . . . . . . 47

4.3 Summary of pion data for inner points . . . . . . . . . . . . 47

5.1 Calculated $\mathrm{ADC}$ to $\mathrm{GeV}$ conversion values from electron data . . . . . 62

7.1 Summary of stochastic and constant term fit results based on modular weights . . . . . . . . . . . . . . . . . . . 88

9.1 Noise-subtracted energy resolution results: 4L $\pi$ data, cylindrical clustering 120

9.2 Fit results for noise-subtracted energy resolution: $4 \mathrm{~L} \pi$ data, cylindrical clustering . . . . . . . . . . . . . . . . . . . 122

9.3 Noise-subtracted energy resolution results: $4 \mathrm{~L} \pi$ data, topological clustering 124

9.4 Fit results for noise-subtracted energy resolution: $4 \mathrm{~L} \pi$ data, topological

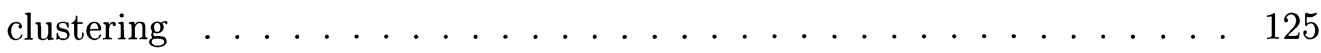




\section{List of Figures}

1.1 The Standard Model particles and force mediators . . . . . . . . . . . . 3

1.2 The LHC experiments . . . . . . . . . . . . . . . . . 6

1.3 The ATLAS Detector . . . . . . . . . . . . . . . 7

1.4 Simulated tracks showing the various sub-components of the ATLAS detector 10

2.1 Total photon cross-sections for photons incident a lead target . . . . . . 18

2.2 Processes for electron energy loss as a function of energy . . . . . . . . . 19

2.3 Depiction of the development of an electromagnetic shower . . . . . . . . 23

3.1 The ATLAS calorimeter system . . . . . . . . . . . . . . . 31

3.2 A side view of one of the ATLAS forward calorimeters . . . . . . . . 33

3.3 The forward calorimeter electrode and readout channel structure . . . . . 35

3.4 A sample liquid argon ionization pulse . . . . . . . . . . . . 36

4.1 The 2003 FCal test beam configuration . . . . . . . . . . . . . . . . 40

4.2 The five selected beam impact points for the FCal test beam . . . . . . . 44

5.1 Active readout channel regions during the FCal test beam data collection 52

5.2 The topological clustering algorithm . . . . . . . . . . . 55

5.3 Representation of the cylindrical and topological clustering algorithms for the FCal . . . . . . . . . . . . . . . . . . . . 55

5.4 Reconstructed energy distribution for $200 \mathrm{GeV}$ pions at the EM scale . . 58

6.1 Function to be minimized for modular weight extraction . . . . . . . 73 
6.2 Extracted 4 L modular weights: cylindrical clustering . . . . . . . . . . 75

6.3 Extracted $4 \mathrm{~L}$ modular weights: topological clustering . . . . . . . . . 76

7.1 Reconstructed energy distributions for $4 \mathrm{~L} \pi$ data with modular weights applied: cylindrical clustering . . . . . . . . . . . . 81

7.2 Reconstructed energy distributions for $4 \mathrm{~L} \pi$ data with modular weights applied: topological clustering . . . . . . . . . . . . 82

7.3 Summary of linearity plots with and without application of modular weights 84

7.4 Noise distributions with $200 \mathrm{GeV}$ modular weights applied: cylindrical

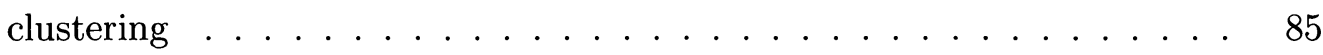

7.5 Comparison of noise RMS values as a function of energy . . . . . . . . 86

7.6 Summary of noise-subtracted energy resolution results, with and without the application of modular weights . . . . . . . . . 87

8.1 Visual representation of radial binning of cells for the radial setup . . . . 92

8.2 Radial profile for the number of included cells: point 4L data . . . . . . 96

8.3 Radial profile for the number of included cells: inner point data . . . . . 97

8.4 EM scale energy density radial profiles for $4 \mathrm{~L} 200 \mathrm{GeV}$ pion data . . . . 100

8.5 $200 \mathrm{GeV}$ radial weights extracted from point 4L: cylindrical clustering, standard setup . . . . . . . . . . . . . . . . . 104

8.6 $200 \mathrm{GeV}$ radial weights extracted from point 4L: topological clustering, standard setup . . . . . . . . . . . . . . . . . 105

8.7 Energy binning used in the radial setup . . . . . . . . . . . . 107

$8.8200 \mathrm{GeV}$ radial weights extracted from point 4L: cylindrical clustering, simplified setup . . . . . . . . . . . . . . . . . . 108

$8.9200 \mathrm{GeV}$ radial weights extracted from point 4L: topological clustering, simplified setup . . . . . . . . . . . . . . . . . . 108

8.10 Geometry for determining cluster centre impact positions . . . . . . . . . 110

8.11 Comparison between projected impact positions on FCal 1 . . . . . . . 113

$8.12200 \mathrm{GeV}$ radial weights extracted from point 4L: topological clustering, simplified setup with no tracking . . . . . . . . . . . . . . 114 
8.13 Extra clusters generated in the forward calorimeter . . . . . . . . . . 115

9.1 Linearity comparison between weighting schemes: cylindrical clustering . 118

9.2 Linearity comparison between weighting schemes: topological clustering . 119

9.3 Noise-subtracted energy resolution comparison between weighting schemes: cylindrical clustering . . . . . . . . . . . . . . . . . . . . . . . . 121

9.4 Noise-subtracted energy resolution comparison between weighting schemes: topological clustering . . . . . . . . . . . . . . . . . . . . 123

9.5 Comparison of reconstructed energy distributions with and without hadronic weights applied . . . . . . . . . . . . . 126

10.1 Chosen beam impact points for the test beam setup . . . . . . . . . . 128

10.2 Weighted and unweighted reconstructed energy radial profiles . . . . . . 131

10.3 Comparison of weighted and unweighted reconstructed energy radial profiles 133

A.1 Extracted 4H modular weights: cylindrical clustering . . . . . . . . . . . 140

A.2 Extracted $4 \mathrm{H}$ modular weights: topological clustering . . . . . . . . . . 140

A.3 Reconstructed energy distributions for $4 \mathrm{~L} \pi$ data with modular weights applied: no clustering . . . . . . . . . . . . . . . . . . . . . . . . . . . . 142

A.4 Noise distributions for $4 \mathrm{~L} \pi$ data with modular weights applied: no clustering 143

A.5 $200 \mathrm{GeV}$ radial weights extracted from point 1: topological clustering, standard setup . . . . . . . . . . . . . . . . . . . . . . . . 145

A.6 $200 \mathrm{GeV}$ radial weights extracted from point 2: topological clustering, standard setup . . . . . . . . . . . . . . . . . . . . . 145

A.7 $200 \mathrm{GeV}$ radial weights extracted from point 3: cylindrical clustering, standard setup . . . . . . . . . . . . . . . . . . . . . . 146

A.8 $200 \mathrm{GeV}$ radial weights extracted from point 3: topological clustering, standard setup . . . . . . . . . . . . . . . . . 146

A.9 $200 \mathrm{GeV}$ radial weights extracted from point $4 \mathrm{H}$ : cylindrical clustering, standard setup . . . . . . . . . . . . . . . . . . . 147

A.10 $200 \mathrm{GeV}$ radial weights extracted from point $4 \mathrm{H}$ : topological clustering, standard setup . . . . . . . . . . . . . . . . . . . . . . 147 
A.11 $200 \mathrm{GeV}$ radial weights extracted from point 3: cylindrical clustering, simplified setup . . . . . . . . . . . . . . . . . 148

A.12 $200 \mathrm{GeV}$ radial weights extracted from point 3: topological clustering, simplified setup . . . . . . . . . . . . . . . . . . 148

A.13 $200 \mathrm{GeV}$ radial weights extracted from point $4 \mathrm{H}$ : cylindrical clustering, simplified setup . . . . . . . . . . . . . . . . . 149

A.14 $200 \mathrm{GeV}$ radial weights extracted from point $4 \mathrm{H}$ : topological clustering, simplified setup . . . . . . . . . . . . . . . . . 149

A.15 Comparison of weighted and unweighted reconstructed energy $\eta$-profiles . 150

B.1 Binning of cells for the radial setup . . . . . . . . . . . . . . . . 152 


\section{Glossary of Terms}

- ALICE - A Large Ion Collider Experiment: one of four main LHC experiments

- ATLAS - A Toroidal LHC ApparatuS: one of four main LHC experiments

- BPC - Beam Positioning Chamber

- CEDAR - ČErenkov Differential Counter with Achromatic Ring Focus

- CERN - European Centre for Nuclear Research

- CMS - Compact Muon Solenoid: one of four main LHC experiments

- FCal - Forward Calorimeter

- LAr - Liquid Argon

- LEP - Large Electron-Positron Collider

- LHC - Large Hadron Collider

- LHCb - LHC-beauty: one of four main LHC experiments

- PEEK - PolyEther Ether Ketone

- SCT - Semi-Conductor Tracker

- TRT - Transition Radiation Tracker 


\section{Chapter 1}

\section{Introduction and Motivation}

\subsection{General Introduction}

Since its inception, the European Centre for Nuclear Research (CERN), located near Geneva, Switzerland, has been recognized as one of the leading research institutions for its preeminent role in fundamental particle physics research and its innovation of experimental methods, software design and detector technology. Such a statement is particularly true at the time of writing: the end of 2008 saw the culmination of many years of planning, development and construction as the first beams of protons with energies in the TeV range were successfully circulated by means of CERN's Large Hadron Collider (LHC), with the first collisions taking place in 2009.

As one of the main LHC experiments, the colossal ATLAS experiment (A Toroidal LHC ApparatuS), will probe physics at the smallest scale as the high energy proton collisions at its centre recreate conditions as they were in the universe only a tiny fraction 
of a second after the Big Bang. The so-called Standard Model of Particle Physics, the model which governs the rules with which the universe's most fundamental building blocks interact and one of the most scrutinized and successful theories of present-day science, will be pushed to its limits in an effort to complete the pieces of one of nature's most elusive puzzles concerning the nature of the universe and the origin of matter.

It is indeed an exciting time for particle physics research, as CERN once again reclaims its place at the forefront of particle physics research, in what is sure to be a decade of scientific discovery, fulfillment, new challenges and - if we are so fortunate - surprises.

\subsection{The Standard Model of Particle Physics}

There are relatively few theoretical models which have shared a level of success similar to that of the Standard Model. The initial suggestion that the protons and neutrons making up an atomic nucleus are themselves made up of yet smaller, more fundamental building blocks was met with strong resistance. Such resistance to this and other principal tenets of the Standard Model were by this point to be expected however, given the same initial reactions to some of the major theories since the quantum revolution at the turn of the century. It could be argued that such initial misgivings only served to heighten one's appreciation for the triumph of the Standard Model, which has repeatedly been shown to accurately model the universe at the smallest scale. Figure 1.1 shows a summary of the twelve fermions - six quarks and six leptons - as well as the four gauge bosons which make up the constituents of the Standard Model. The model assumes that these sixteen particles, along with their antimatter counterparts, are not themselves composed 
of smaller pieces - that they are truly fundamental.

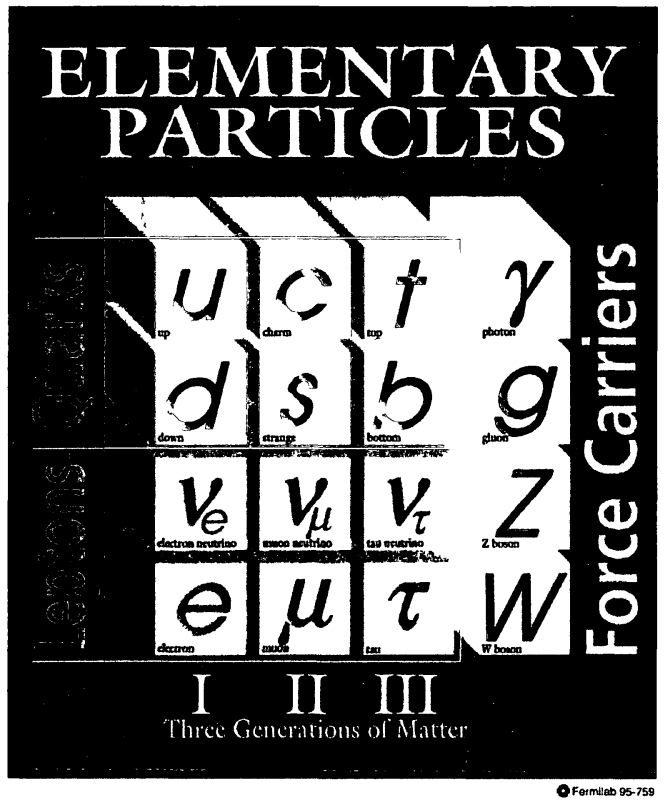

Figure 1.1: The six quarks, six leptons and four gauge bosons which constitute, along with the antimatter counterparts of both the quarks and leptons, the fundamental building blocks of the universe as represented by the Standard Model of particle physics [1].

It is hoped that the question as to the seemingly arbitrary assignment of mass to each of these fundamental particles will soon be addressed as physicists strive to produce and identify the so-called Higgs boson - the missing piece of the Standard Model which is hypothesized to generate the mass of the fundamental particles by means of a process referred to as the Higgs mechanism, via spontaneous symmetry breaking.

Yet despite its tremendous success, the Standard Model is known to be an incomplete theory even if indisputable evidence that the Higgs boson exists were to be found. While 
some discrepancies between model and experiment can be refined with relative ease, such as the addition of mass terms for the neutrinos, the lightest leptons by several order of magnitude which previously and erroneously were thought to be massless, other discrepancies blemish the model's otherwise unparalleled track record. Among the model's top deficiencies are its inability to explain the matter-antimatter asymmetry in the universe and to account for the overwhelmingly high fraction of dark energy and dark matter in the universe, in addition to being able to state precisely what each of these constituents might be.

Whether or not the Standard Model, as it exists today, is sufficient to interpret the results of analyses of ATLAS data remains to be seen. Should it prove inadequate, there are a plethora of competing theories which vie to take its place as the model which best describes the universe with all its peculiarities. One of the strongest contending theories, Supersymmetry (SUSY), suggests that for each of the current particles constituting the Standard Model there exists a superpartner whose intrinsic quantum mechanical spin differs from its Standard Model counterpart by a value of $\frac{1}{2}$. In this way, the number of predicted fundamental particles which make up the universe would immediately double in size.

For the ATLAS experiment, successfully weeding through the proposed theories and searching for answers to the above questions about the nature of the universe, will require a concerted effort from the entire ATLAS team. It will be important to understand the operation of the detector and its components and to develop the necessary tools in order to interpret the data.

Before outlining the particular motivation for the analysis summarized in this thesis, 
it is useful to provide information on the background to the analysis, namely that of the LHC and the ATLAS experiment.

\subsection{The Large Hadron Collider at CERN}

Located approximately $100 \mathrm{~m}$ underground in the same tunnel which hosted its predecessor, the Large Electron-Positron Collider (LEP), the LHC accelerates opposing beams of protons around its $27 \mathrm{~km}$ circumference to near luminal speeds. A series of smaller accelerators are used to bring the protons up to an energy of $450 \mathrm{GeV}$. They are then injected into the LHC which ultimately brings the energy of the protons in a given beam to the design value of $7 \mathrm{TeV}$.

The two beams of protons are then made to intersect at four points along the LHC ring, around which the main experiments have been constructed to study high-energy, deeply inelastic collisions of the protons' constituents. The LHC has a design centre of mass energy of $\sqrt{s}=14 \mathrm{TeV}$ and design luminosity [3] of $10^{34} \mathrm{~cm}^{-2} \mathrm{~s}^{-1}$. It should be noted that at the time of writing the centre of mass energy is in fact $\sqrt{s}=7 \mathrm{TeV}$, though it is expected that the design energy will be reached in 2013. Such a high energy is required in order that the cross-sections of interest be large enough, while the high luminosity is required to allow experiments to accumulate enough statistics for the rare processes one hopes to examine.

Figure 1.2 shows the position of the ATLAS detector in the LHC tunnel, along with the other three main experiments: CMS (Compact Muon Solenoid), LHCb (LHCbeauty), and ALICE (A Large Ion Collider Experiment). The tunnel itself contains a 


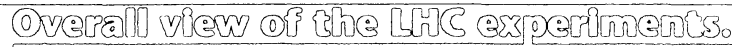

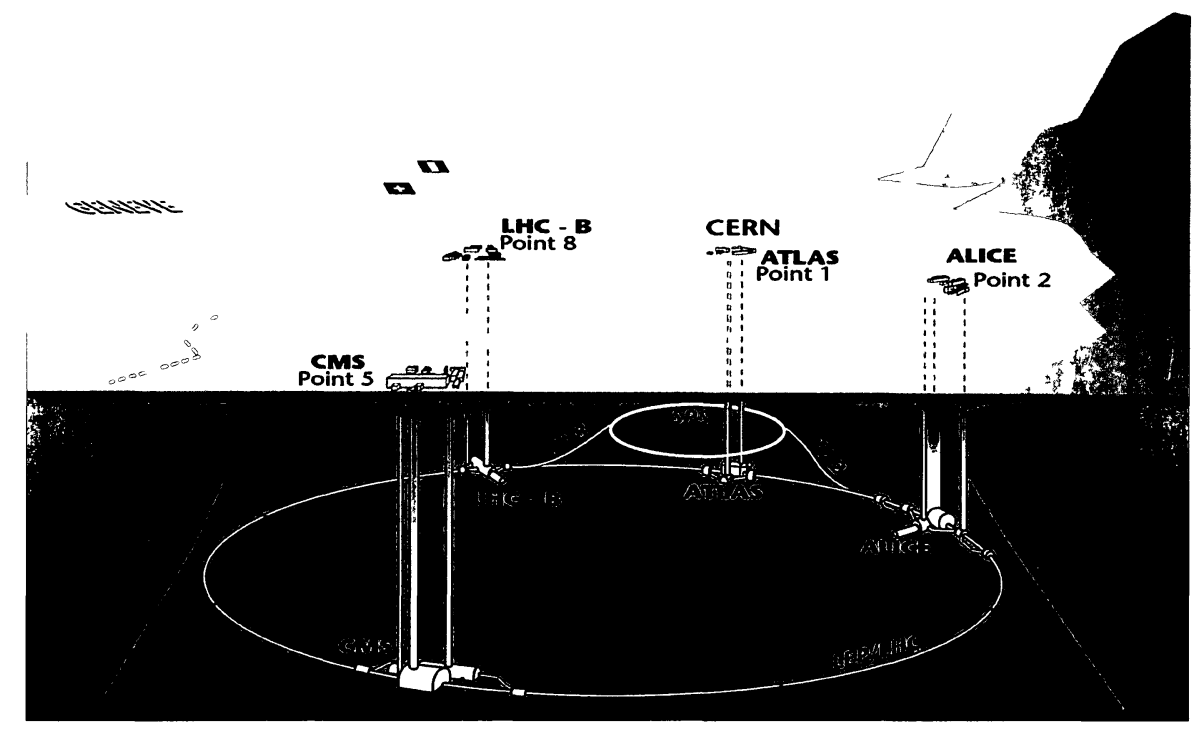

Figure 1.2: A depiction of the LHC tunnel, located approximately $100 \mathrm{~m}$ underground on the French-Swiss border near Geneva, along with the four main experiments situated at the chosen beam intersection points: ATLAS, LHCb, ALICE and CMS [2].

series of large dipole and quadrupole magnets, the former which constrain the proton beam to follow a circular path, and the latter for focusing the beam in the transverse plane. The near-vacuum inside the beam pipe, as well as the extremely low liquid helium temperatures which are required for the superconducting state of the magnets involved, would make for a pleasant environment only for one who is habituated to the far reaches of space. Such conditions are, however, required to allow the circulating beams to travel around the LHC tunnel unimpeded. 


\subsection{The ATLAS Experiment}

It is the role of the LHC, and not of the experiments themselves, to force the opposing beams of protons to cross one another at chosen points for each experiment, causing the collisions between the protons. The particles created during such collisions or their decay products then stream out in many directions from the collision point and interact with the various layers of the detector for the particular experiment. Such is the case for the enormous ATLAS detector, shown in Figure 1.3, which measures approximately $44 \mathrm{~m}$ in length and $25 \mathrm{~m}$ in diameter and has a total mass of nearly 7000 metric tons.

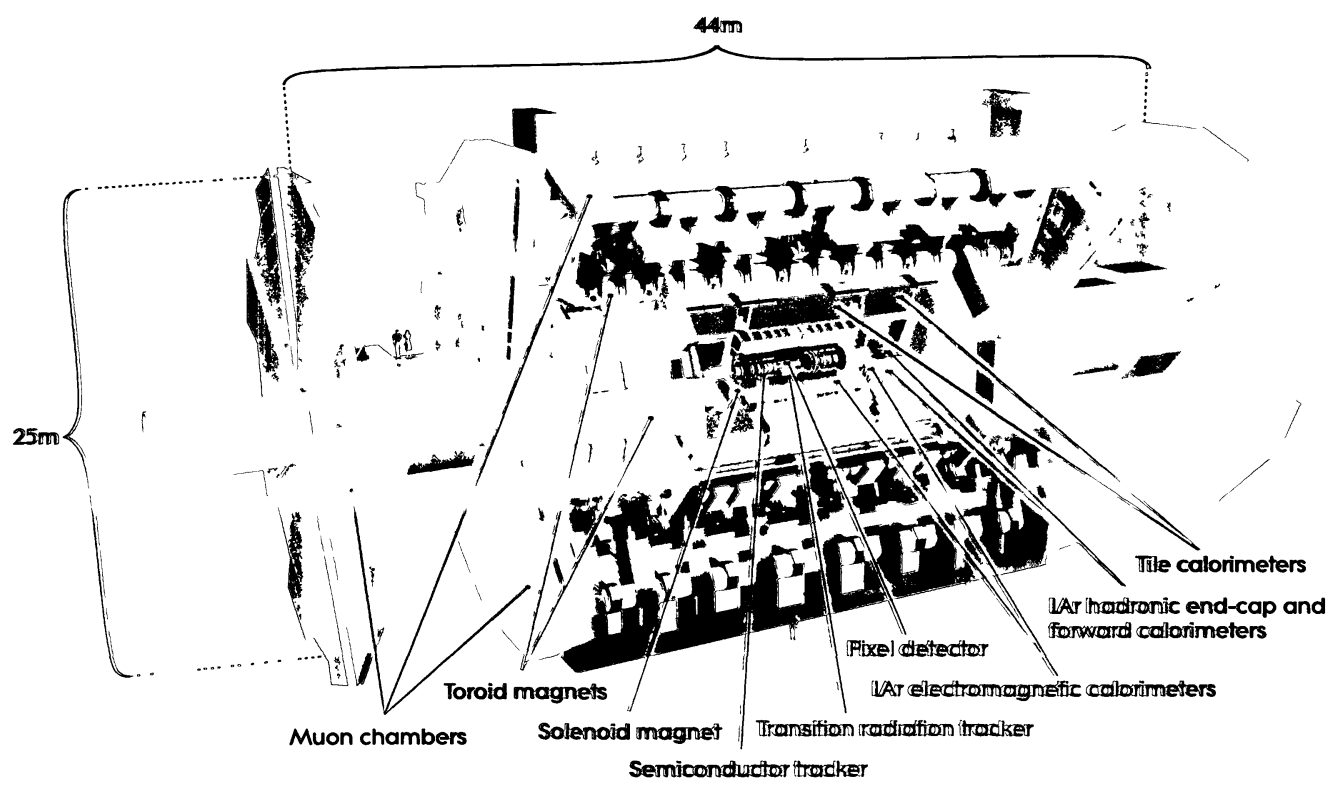

Figure 1.3: A computer generated cut-away view of the entire ATLAS detector along with labels of its various components [2]. 
In reality of course, the ATLAS detector, like the detectors of the other main LHC experiments, does more than simply act as a means of hermetic containment for highenergy particles: the ATLAS detector itself is composed of distinct layers of sub-detectors, each allowing for specific measurements, which are then used in combination in order to provide as much information as possible about the fundamental reactions taking place at the interaction point - the point where the LHC beams are made to cross one another at the centre of the detector.

A visual representation of the various components of the ATLAS detector is shown in Figure 1.4, along with the key signatures exhibited by some of the common types of particles which are seen as a result of collisions. This diagram shows a cross-sectional slice through the centre of the detector and illustrates the layering of the various detector components with increasing radial distance from the interaction point. The detector consists of three principal parts, namely the inner tracking region, the calorimeter system and finally the muon spectrometer. The composition and function of each of these parts are summarized very briefly below:

- The inner tracking region of the ATLAS detector consists of the Pixel detector, the Semi-Conductor Tracker (SCT) detector and the Transition Radiation Tracker (TRT). The Pixel and SCT detectors provide high-precision measurements of the positions of charged particles in order to reconstruct a given particle's trajectory. This information is then used to determine particle momenta and to reconstruct primary and secondary vertices - the positions in space at which a particle of interest decayed to two or more particles. The large solenoidal magnet surrounding this inner region provides a nominal $2 \mathrm{~T}$ field which bends the paths of such charged 
particles, thereby facilitating a high-precision momentum measurement. The TRT utilizes layers of gas-filled straw tube elements to further determine the tracks of particles, enhance momentum resolution and provide a means of electron identification complimentary to the calorimeter system [4].

- The calorimeter system for the ATLAS detector, which can be subdivided into the electromagnetic or EM calorimeter and the hadronic calorimeter, acts to measure the energy of particles within its volume.

- Encompassing these two inner regions is the immense muon spectrometer system which is designed to measure, with the aid of the large toroidal magnets, the momentum of muons. Muons interact minimally with the calorimeter system and as such are able to travel to great distances through the entire detector. 


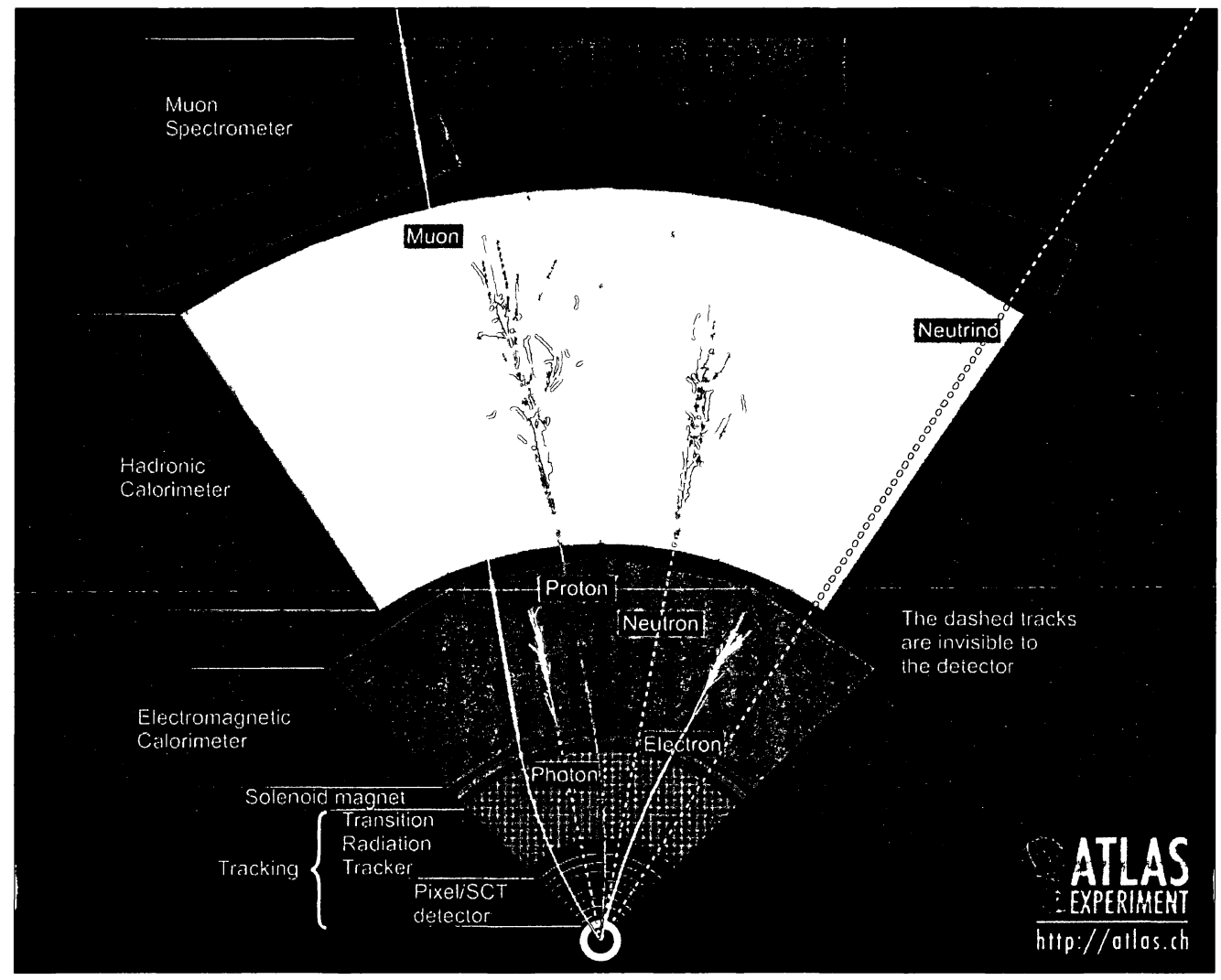

Figure 1.4: A representation showing a cross-sectional view of the entire ATLAS detector at the centre of its barrel region, in addition to the signals one expects to see in each of the detector's key regions as a result of proton collisions at the interaction point [2]. 


\subsubsection{The ATLAS Coordinate System}

The ATLAS detector utilizes a cartesian coordinate system, though in actuality one refers more often to other coordinates which will be presented below. Based on the detector's position within the LHC tunnel, the positive $x$ direction is such that it always points radially inward towards the centre of the LHC ring - some $4.3 \mathrm{~km}$ away from the centre of the LHC beam pipe. The positive $y$ direction points upward at a right angle with respect to the slightly tilted plane of the LHC ring. The positive $z$ direction is chosen in order to make a right-handed system, i.e. such that $\hat{x} \times \hat{y}=\hat{z}$.

The azimuthal angle $\phi$ is measured as the angular separation from the positive $x$-axis towards the positive $y$-axis. The polar angle $\theta$, defined as the angle between a vector and the positive $z$-axis, is typically replaced, due to relativistic considerations, with the variable $\eta$, referred to as the pseudorapidity, which is related to the polar angle as shown in Equation (1.1) below:

$$
\eta=-\ln \left(\tan \frac{\theta}{2}\right)
$$

The momentum and energy of particles are both important variables within the ATLAS experiment, with the energy being the primary quantity of interest for the present thesis. One will also customarily speak of transverse momentum and transverse energy. Momentum, familiar as a vector quantity, will in general have three non-zero components into which it can be decomposed, namely $p_{x}, p_{y}$ and $p_{z}$ corresponding to each of the directions recently presented. The transverse momentum, written $p_{T}$, corresponds to the magnitude of the momentum in the plane transverse to the z-axis, such that: 


$$
p_{T}=\sqrt{p_{x}^{2}+p_{y}^{2}}
$$

The protons colliding at the centre of the ATLAS experiment are composed of partons, which carry varying fractions of a given proton's momentum. The collisions themselves are between these partons, and head-on collisions are more likely to be of interest since there is then a maximal amount of collision energy available to produce new particles. Energy, though not a vector quantity, can be described by using the momentum vector to calculate a component in the transverse, or $x-y$ plane, and which is referred to as the transverse energy, $E_{T}$. Interesting events with a large total transverse energy are easier to separate out in the experimental data from relatively uninteresting background events.

\subsection{Motivation for the Present Analysis}

Given the nature of the ATLAS experiment, one can recognize that its ultimate success relies on the individual performance of its constituent parts. As such, the present analysis is a contribution to the ongoing effort to calibrate a portion of the ATLAS calorimeter system, namely the Forward Calorimeter (FCal) which will be presented in more detail in Chapter 3.

To this end, a set of test beam data has been analyzed, which was taken in a beam test of the forward calorimeter carried out in 2003. The present analysis focuses on the pion data from the test beam in an effort to study the results and feasibility of applying different hadronic weighting schemes to such data. The forward calorimeter system differs greatly from the other parts of the ATLAS calorimeter system both in its 
design and the degree to which it will be irradiated by high-energy particles. Like the other sections of the calorimeter system, the FCal will require the application of hadronic weights to its data in order to account for its non-compensating nature as a calorimeter. While such weights are applied in a standard present-day analysis of ATLAS data for the different regions of the calorimeter, it is unlikely that the current weights are optimized for the forward region and it is important to continually scrutinize the state of the current algorithms to see if improvements can be made.

The focus of the present analysis is on the derivation and application of hadronic weights which attempt to exploit the transverse physical characteristics of hadronic shower development. The goal is to develop an algorithm or to refine the present algorithm for hadronic weighting. The pertinent benchmark for the success of a new scheme is an improvement in the energy resolution which is a measure of precision in energy reconstruction, while at the same time ensuring an accurate energy response for the calorimeter.

The improvement in the energy measurement within the forward calorimeter system, or the precision thereof, will be of great interest to those involved in the many analyses of ATLAS data which require calorimeter coverage that includes the high- $\eta$ region close to the LHC beam line. 


\section{Chapter 2}

\section{Calorimetry in Collider Experiments}

\section{$2.1 \quad$ Introduction}

The central region of a colliding experiment's detector is teeming with high-energy particles. Left to their own devices and unimpeded by any force or body, such particles or those into which they decay will travel great distances until they reach a volume of material into which they can deposit their energy. The primary purpose of any calorimeter system is to measure the energy of such particles accurately and precisely. In order to do so, it is first necessary that such energy be deposited in the material of the calorimeter.

A high-energy particle deposits its energy in a number of ways as will be expanded upon throughout this chapter. In fact, a single particle will often initiate a cascade or shower of particles which act to spread out the energy deposit in space. The idea of a shower plays a key role in the understanding of the design of a calorimeter, and will thus be referred to often in the subsequent sections. The properties of such showers and the 
way in which they develop is explained in further detail in Sections 2.4 and 2.5.

One of the types of material constituting a significant part of the calorimeter is generally referred to as the absorbing material since a significant fraction of a particle's energy is deposited within its volume. It is customary to use very dense materials ${ }^{1}$ such as copper, lead or tungsten for this purpose which more effectively limit the distance over which the particles can travel.

Such an absorbing material on its own, however, would not provide one with the desired measurement of a given particle's energy. Active materials, in contrast, are typically far less dense than typical absorbing materials and many take advantage of the fact that the total ionization produced in a material by an incident particle's shower is proportional to its energy. A measure of this ionization thus allows the energy of the incident particle to be calculated.

\subsection{Sampling Calorimeters}

The previous section introduced the two types of materials common to the design of calorimeters: absorbing and active materials. In certain calorimeters, one material can satisfy the requirements of both an absorbing and active material, namely that of absorbing the energy of particles, and of making a post hoc measurement of the particle's energy just prior to its contact with the calorimeter system. Such calorimeters are classified as homogeneous in that they consist uniquely of one type of material. The limited number of different materials that can effectively be used for a homogeneous calorimeter

\footnotetext{
${ }^{1}$ The pertinent variable is in fact the radiation length, to be introduced in section 2.3
} 
however, as well as the associated cost of such materials, makes such a design impractical for certain experiments. ATLAS is an example of such an experiment. The high level of particle fluxes to which the ATLAS calorimeters are exposed would not allow such homogeneous materials to survive. For other cases, however, the advantage of a homogeneous calorimeter is that one is able to actively sample the energy deposited throughout the entirety of the calorimeter.

Another possibility is to place alternating layers of both active and absorbing material such that particles will cross a great number of boundaries between the materials; such a particle will therefore both deposit a significant amount of energy in the absorbing material which will limit its depth, and provide an energy measurement in the active material. Calorimeters in which this is done are referred to as sampling calorimeters, and have the obvious disadvantage that only a fraction of the energy is measured or sampled by the active material. The calorimeter must then be calibrated with incident particles of known energy - also a necessary step in the case of homogeneous calorimeters. The ATLAS forward calorimeter system, which is the focus of the present analysis and the design of which will be described in the following chapter, is an example of a sampling calorimeter. Sampling calorimeters are in fact the standard type of calorimeters used in all modern hadron collider experiments. 


\subsection{Interactions of Particles within the Calorimeter}

Of specific importance to the field of sampling calorimetry is the way in which highenergy electrons and positrons, as well as photons, deposit their energy as they interact with the material of a calorimeter. A photon can undergo one of several processes, where the crucial quantity of interest which determines the most likely process and therefore the most statistically probable is the cross-section of a given process, typically measured in barns, where:

$$
1 \text { barn }=10^{-28} \mathrm{~m}^{2} \text {. }
$$

The cross-sections for each type of interaction vary greatly with energy. A comparison between model and experimental data [5] is shown in Figure 2.1 as an example of this variation for the specific case of photons travelling within bulk lead.

One can see from this figure that for photons with energy greater than $10 \mathrm{MeV}$, the dominant process is of pair production in the presence of a nuclear field, in which the photon interacts with a virtual photon in the field of the nucleus to produce both an electron and a positron.

Figure 2.2 shows the fractional energy loss for electrons or positrons travelling through lead, as a function of their energy. Again, one sees that certain energy loss mechanisms are more significant for different energy scales. The dominant mechanism for energy loss for both electrons and positrons at high energies is that of brehmsstrahlung radiation, in which the acceleration of the electron or positron causes the emission of a photon. In the low energy regime, the process by which energy is expended by the electron or positron in order to free another electron bound to a parent nucleus dominates - 


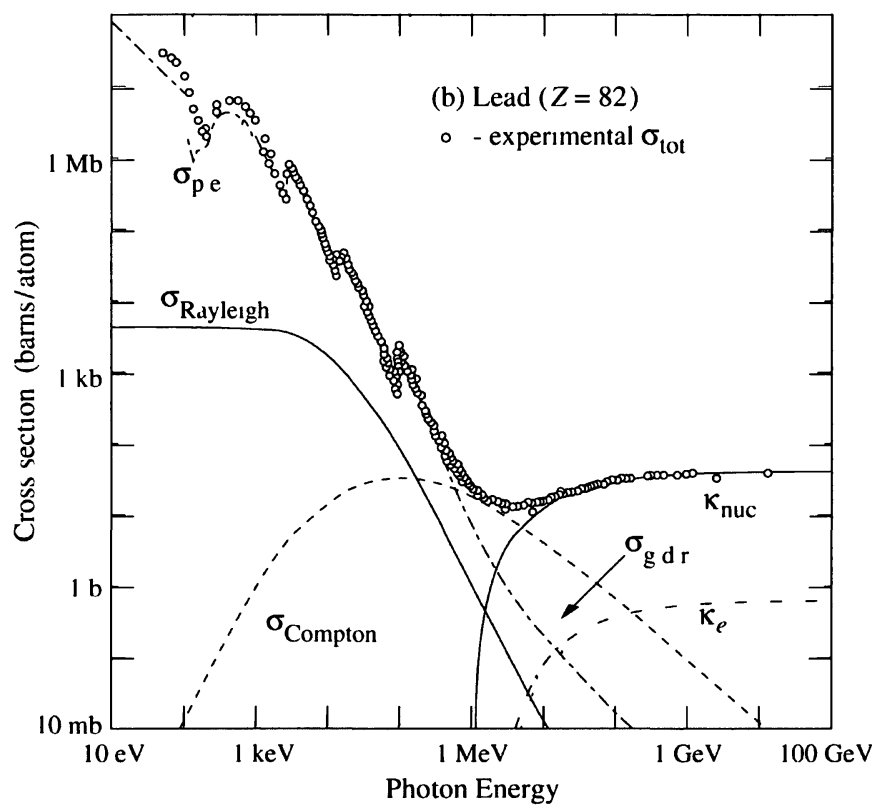

Figure 2.1: The photon total cross-sections shown as a function of the photon energy, as well as the particular cross-sections for individual processes of interest, namely: the photoelectric effect $\left(\sigma_{\mathrm{p} \mathrm{e}}\right)$, Compton and Rayleigh scattering $\left(\sigma_{\text {Compton }}\right.$ and $\left.\sigma_{\text {Raylegh }}\right)$, photonuclear interactions in which a target nucleus is broken up $\left(\sigma_{\mathrm{g} \mathrm{d} \mathrm{r}}\right)$, and pair production in the presence of either a nuclear or electron field $\left(\kappa_{\text {nuc }}\right.$ and $\left.\kappa_{\mathrm{e}}\right)$. [5]

the aforementioned process known as ıonızatıon. Electrons or positrons can also lose energy vıa scattering processes, referred to as Møller and Bhabha scattering, respectively. Finally, the analogous process to the pair production mechanism for photons is that of pair annıhılatıon, whereby a positron with sufficiently low energy will annihilate with a target electron resulting in the creation of two characteristic $511 \mathrm{keV}$ photons.

A useful length scale in this context is the mean distance an electron or positron must travel through a given material in order that its energy be reduced to $1 / e$ of its 


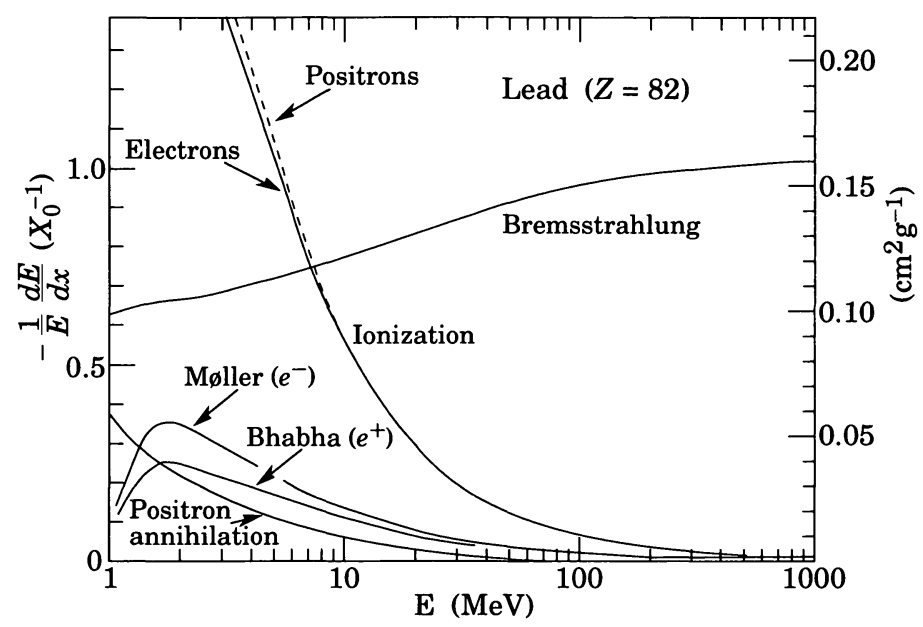

Figure 2.2: The fractional energy loss for electrons for various processes are shown as a function of electron energy. [5]

original value. Such a distance, typically measured in units of $\mathrm{g} / \mathrm{cm}^{2}$, is referred to as the radiation length of the material, $X_{0}$, whose value can be determined approximately by the following [5]:

$$
X_{0}=\frac{716.2 A}{Z(Z+1) \ln (287 / \sqrt{Z})}
$$

The terms $Z$ and $A$ present in Equation (2.2) correspond to the atomic number and atomic mass of the absorbing material, respectively, where the latter is assumed to be in units of $\mathrm{g} / \mathrm{mol}$. The units for the multiplicative constant in Equation (2.2) are such that the calculated value for $X_{0}$ will be in units of $\mathrm{g} / \mathrm{cm}^{2}$. Due to the interplay between $Z$ and $A$ for a given element, the overall effect is that the radiation length will tend to decrease with increasing $Z$. For equal volumes of absorbing materials then, the material with a higher atomic number $Z$ will have a smaller associated radiation length, and will thus be 
better suited to contain high-energy particles and the showers that result from them. It is therefore an important factor in the design of a calorimeter, insofar as a calorimeter's potential physical size is generally of considerable consequence.

For a given material composed of $i$ distinct materials, each of which with radiation length $X_{\imath}$ and contributing a fraction $V_{\imath}$ of the total volume, the effective radiation length $X_{e}$ can be expressed by Equation (2.3) below [6]:

$$
X_{e}=\sum_{\imath} V_{\imath} / X_{\imath}
$$

Based on the energy loss mechanisms presented thus far, one can immediately recognize the interplay between the dominant interaction mechanisms of high-energy photons, electrons and positrons. In addition to reducing the energy of the original particle of interest, pair production and brehmsstrahlung cause the initial particles to spawn additional particles. In this way, the number of particles responsible for energy dispersion increases rapidly. Such an escalation in the number of constituent particles based on an initial electron, positron or photon, is known as an electromagnetıc shower or electromagnetıc cascade. As it turns out, electromagnetic showers have many interesting physical characteristics which can be exploited in the calorimeter design and in understanding the measurement provided by a given calorimeter. 


\subsection{Properties of Electromagnetic Showers}

Electromagnetic showers stem from an initial high-energy photon, electron or positron undergoing the process of pair production or brehmsstrahlung. This results in a bifurcation of sorts, in which one initial particle now becomes two; each of these particles in turn produces additional particles as it continues to deposit energy by various other means while traversing the calorimeter material. A type of chain reaction ensues, with a rapid increase in the number of particles, though it is recognized that the total energy for all shower particles continues to decrease as the shower propagates through a material. As one might expect, during the initial part of a shower the total number of shower particles increases exponentially.

A simple model developed by W. Heitler [7], provides insight into the longitudinal development of electromagnetic showers. A shower which began as a single particle can be expected to contain $N(t)$ particles after a distance $t$ in radiation lengths, where:

$$
N(t)=e^{t \ln 2}=2^{t}
$$

If the initial energy of the primary particle were $E_{0}$, where it is assumed that this value is far greater than the average energy loss per radiation length, then the average energy of each particle after the same distance $t$, would be:

$$
E(t)=E_{0} / 2^{t}
$$

Equations (2.4) and (2.5) are not entirely surprising based on the definition of the 
radiation length, $X_{0}$; perhaps less intuitive is the result that a shower contains a maximum number of particles at a depth $t_{\max }$, again in radiation lengths, where [7]:

$$
t_{\max }=\frac{\ln \left(E_{0} / E_{C}\right)}{\ln 2}
$$

In Equation (2.6), $E_{C}$ is the critical energy, defined to be the energy at which a charged particle's energy loss due to radiation in a given material is equal to its energy loss due to ionization. Assuming the atomic number of the medium to be $Z$, where $m_{e} c^{2}$ is the electron rest mass in $\mathrm{GeV}$, the critical energy is given by [8]:

$$
E_{C}=\frac{1600}{Z} m_{e} c^{2}
$$

Such a model provides only a rough approximation however; in practice the sharp peak occurring at $t_{\max }$ is smoothed out.

Beyond a depth of $t_{\max }$, the electromagnetic shower quickly loses its energy by the various mechanisms described in the preceding section. It has been pointed out by $R$. Wigmans [6], that the value of $t_{\max }$ will increase for showers developing in materials with larger atomic numbers, $Z$, and that furthermore showers in such materials will develop more slowly beyond a distance of $t_{\max }$.

Due to the fact that photons will only convert after having travelled an average distance of 1.3 radiation lengths [8] through a given material, they will generally initiate an electromagnetic shower at a greater depth. This fact is taken into account by typically referring to the absorption length $X_{P}$ in the case of photons, where $X_{P}=\frac{9}{7} X_{0}$.

In terms of lateral or transverse properties of electromagnetic showers, the shower 
will be mostly contained within a cylinder with a radius of one radiation length prior to the shower maximum depth [8], given in Equation (2.6). After this point, the radius of the cylinder required to contain $95 \%$ of the shower with respect to its central axis is roughly equal to twice a quantity referred to as a Molière radius, $R_{\mathrm{M}}$, given in units of $\mathrm{g} / \mathrm{cm}^{2}$, with a value given by [9]:

$$
R_{\mathrm{M}}=7\left(\frac{A}{Z}\right)
$$

Figure 2.3 shows a depiction of the development of an electromagnetic shower, where one radiation length has been shown which provides a scale for the shower's depth in a material.

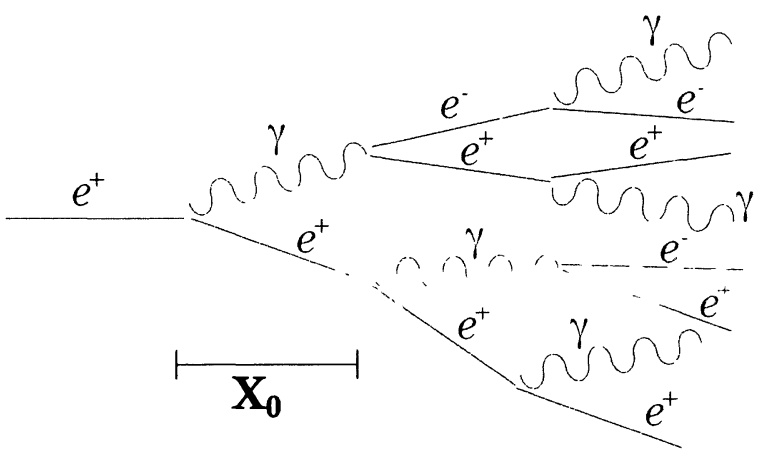

Figure 2.3: The depiction of the development of an electromagnetic shower initiated in this case by a positron. Also shown is the radiation length, $X_{0}$, which is used to quantify the depth of such showers. 
In summary then, one can see that the depth of an electromagnetic shower into a material varies logarithmically with the incident particle energy, and that $95 \%$ of the shower's energy should be fully contained laterally within $2 R_{\mathrm{M}}$ of the shower axis. Having laid down the principal properties of interest for the case of electromagnetic showers, what remains for the present chapter is to highlight the properties of yet another type of shower - hadronic showers. As their name implies, hadronic showers are those initiated by high-energy hadrons, and though they share some general characteristics with their electromagnetic counterparts, hadronic showers are manifestly different in the way that they behave; it is these differences which will feature in the motivation for the present study, particularly in regards to hadronic calibration of calorimeters.

\subsection{Properties of Hadronic Showers}

A hadronic particle with sufficient energy can initiate a shower or cascade, as with electrons, positrons and photons. In a hadronic shower typically half of the hadronic particle's energy is transferred to fast secondary hadrons [9]. Such secondary hadrons also acquire a significant amount of transverse momentum, giving rise to the first physical characteristic of hadronic showers: they tend to be more spread out laterally.

The production of neutral pions $\left(\pi^{0}\right)$, which almost exclusively decay to two photons, will give rise to the initiation of electromagnetic showers as summarized in the previous section. Since $\pi^{0}$ production is a common feature of hadronic showers, it follows that hadronic showers themselves contain a number of electromagnetic showers. The proportion of electromagnetic shower within a hadronic shower changes with energy, and is of 
importance to the calibration of a calorimeter.

The longitudinal scale of hadronic shower development makes use of a quantity $\lambda_{I}$, referred to as the nuclear absorption length or nuclear interaction length. This quantity is larger than the radiation length $X_{0}$ for a given material, and the mean depth of a hadronic shower, given an initial hadron of energy $E_{0}$, can be parameterized as [10]:

$$
\lambda_{\max }=0.90+0.36 \ln E_{0}
$$

The value of $\lambda_{\max }$ in Equation (2.9) is given in units of nuclear interaction lengths, and one can again observe that the depth of a hadronic shower increases logarithmically with increasing hadron energy. The depth of material required to contain $90 \%$ of a hadronic shower in nuclear interaction lengths can also be parameterized as [10]:

$$
L(90 \%)=-1.26+1.74 \ln E_{0}
$$

At this point it is useful to deal with concrete examples of materials to develop a sense of scale for the two quantities referred to as the radiation length and the nuclear interaction length. It is advantageous to use copper $(\mathrm{Cu})$ and tungsten $(\mathrm{W})$ for reasons which will become apparent in the following chapter.

Table 2.1 summarizes the quantities of interest for these two materials, based on values from the 2008 publication of data from the Particle Data Group table of atomic and nuclear properties of materials [5]. The radiation length and nuclear interaction length for each material are both expressed in units of $\mathrm{g} / \mathrm{cm}^{2}$, the more common unit for such quantities, and are also expressed in the more physically intuitive units of $\mathrm{cm}$. 
Table 2.1: Summary of nuclear and atomic properties for two chosen elements: copper and tungsten. Values are from the Particle Data Group's 2008 publication [5]. Shown from left to right are the element's atomic number $Z$, density $\rho$, radiation length $X_{0}$, and nuclear interaction length $\lambda_{I}$.

\begin{tabular}{|c|c|c|c|c|c|c|}
\hline Material & $Z$ & $\rho\left[\mathrm{g} / \mathrm{cm}^{3}\right]$ & $X_{0}\left[\mathrm{~g} / \mathrm{cm}^{2}\right]$ & $X_{0}[\mathrm{~cm}]$ & $\lambda_{I}\left[\mathrm{~g} / \mathrm{cm}^{2}\right]$ & $\lambda_{I}[\mathrm{~cm}]$ \\
\hline \hline $\mathrm{Cu}$ & 29 & 8.960 & 12.86 & 1.44 & 137.3 & 15.32 \\
\hline $\mathrm{W}$ & 74 & 19.300 & 6.76 & 0.35 & 191.9 & 9.94 \\
\hline
\end{tabular}

An arbitrarily chosen initial particle energy of $100 \mathrm{GeV}$ should suffice to convince the reader, based on Equations (2.6) and (2.9) in conjunction with the values from Table 2.1 that the longitudinal scale of hadronic showers is significantly larger than that of electromagnetic showers in isolation. In order to develop a sense of scale in terms of shower depths, Table 2.2 summarizes calculated values for three longitudinal variables characterizing electromagnetic and hadronic shower development. Specifically shown are the values of $t_{\max }, \lambda_{\max }$, and $L(90 \%)$ for the case of particles with initial energies ranging from 10 to $500 \mathrm{GeV}$ travelling within a copper or tungsten volume.

Given a hadronic shower and an electromagnetic shower, each initiated by particles of equal energies, the hadronic shower would both penetrate deeper into a material, and would occupy more space laterally. It follows then that another general property of hadronic showers is that they have a lower energy density as compared with electromagnetic showers. This property can be exploited in part to help distinguish a hadronic shower from a purely electromagnetic one, as is presently done for analyses within the ATLAS collaboration. 
Table 2.2: Calculated values for the longitudinal variables characterizing electromagnetic and hadronic shower development. The values for $t_{\max }$ correspond to an initial electron or positron. $\lambda_{\max }$ and $L(90 \%)$ correspond to an initial hadron. The values are shown in $\mathrm{cm}$ for a series of initial energy values $E_{0}$. The material volume is assumed to be either copper or tungsten for which the values of $X_{0}$ and $\lambda_{I}$ can be seen in Table 2.1.

\begin{tabular}{|c|c|c|c|c|c|c|}
\hline \multirow{2}{*}{$\begin{array}{c}\text { Initial Energy } \\
E_{0}[\mathrm{GeV}]\end{array}$} & \multicolumn{3}{|c|}{ Copper } & \multicolumn{3}{c|}{ Tungsten } \\
\cline { 2 - 7 } & $t_{\max }[\mathrm{cm}]$ & $\lambda_{\max }[\mathrm{cm}]$ & $L(90 \%)[\mathrm{cm}]$ & $t_{\max }[\mathrm{cm}]$ & $\lambda_{\max }[\mathrm{cm}]$ & $L(90 \%)[\mathrm{cm}]$ \\
\hline \hline 10 & 12.2 & 26.5 & 42.1 & 3.0 & 17.2 & 27.3 \\
\hline 50 & 15.5 & 35.4 & 85.0 & 3.8 & 22.9 & 55.1 \\
\hline 100 & 17.0 & 39.2 & 103.5 & 4.1 & 25.4 & 67.1 \\
\hline 500 & 20.3 & 48.1 & 146.4 & 4.9 & 312 & 95.0 \\
\hline 1000 & 21.8 & 51.9 & 164.8 & 5.3 & 337 & 106.9 \\
\hline
\end{tabular}

\subsection{Energy Response for Electrons}

The energy response of a calorimeter refers to the value returned for the measurement of a given particle's energy, as it compares with the true energy In the case of a sampling calorimeter $^{2}$, it is not possible to measure the energy deposited in the absorbing material; only the active material provides a signal which can be read out. The relative amounts of absorbing and active material in a given calorimeter are represented by sampling fractions. The sampling fraction is defined to be the amount of energy deposited by

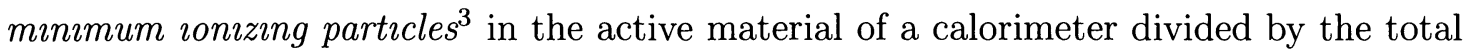
energy deposited by such particles in all regions of the calorimeter [6]. These can vary for

\footnotetext{
${ }^{2}$ Refer to Section 22

${ }^{3} \mathrm{~A}$ minımum lonızıng particle is a partıcle whose rate of energy loss in a material is at a minımum
} 
different regions of the calorimeter depending on its geometric design and composition. Customarily, beams of electrons of known energy are directed towards the calorimeter in question and the measured energy, along with knowledge of the calorimeter's sampling fractions, is used for calibration purposes. The goal of such a calibration is to obtain a calibration constant such that the measured energy times the calibration gives back the true particle energy regardless of the energy, in the case of electrons, positrons and photons. A calorimeter satisfying this requirement is said to have a linear response at the electromagnetic scale.

\subsection{Energy Response for Hadrons}

One would naively hope that a calorimeter with a linear response at the electromagnetic scale would also have a linear response for hadronic particles. In fact, the field of calorimetry would be greatly simplified if such were the case. Unfortunately the response for hadronic particles is lower than that for electrons, and moreover the response is energy dependent, or nonlinear. Further examination of the various energy loss mechanisms for hadronic showers reveals that a fraction of the order of $20 \%$ [11] of a hadronic shower's energy is deposited in the form of nuclear excitations, nuclear breakup, or the recoil of target particles. Given that a calorimeter almost exclusively measures the energy deposited in the form of ionization, this previously mentioned fraction is invisible to the calorimeter and is unsurprisingly often referred to as the invisible energy fraction. It constitutes the largest fraction of a hadronic shower's energy which is not measured by the detector. 
In addition to the invisible energy fraction, the energy carried away by muons and neutrinos constitutes a small, but non-negligible fraction of the total energy of a hadronic shower. This energy is also not detected by the calorimeter, though it does not contribute to the mismeasurement of energy in a significant way ${ }^{4}$.

The fraction $e / h$ is commonly referred to in calorimetry as the ratio of a given calorimeter's response to electrons/positrons $(e)$ over hadronic particles $(h)$. The invisible energy fraction mentioned above which is primarily responsible for the low hadronic response of a calorimeter has been shown to decrease with energy; one can therefore expect the fraction $e / h$ to be largest at lower energies.

In fact, it turns out that there are calorimeters which are designed to compensate either wholly or in part for the invisible energy fractions and are consequently referred to as compensating calorimeters with the goal to have a value of $e / h=1$. The ATLAS calorimeters and the forward calorimeters in particular, however, are non-compensating.

Returning to the primary objectives of a calorimeter then, namely accurate and precise energy measurement, it is clear that such a low and nonlinear energy response for hadronic particles will need to be compensated for in some way; not to do so would result in a severe under-measurement of the energy of any hadronic particles within ATLAS, which is clearly undesirable. Indeed the goal of the present analysis is to determine the optimized scheme for such compensation, while remaining cognizant of the physical properties of both electromagnetic and hadronic showers. Before quantifying such an optimization however, it is first important to examine the design of the particular calorimeter system involved in the present analysis.

\footnotetext{
${ }^{4}$ The energy carried away by muons and neutrinos is on the order of $2 \%$ of a shower's total energy [11].
} 


\section{Chapter 3}

\section{The ATLAS Forward Calorimeters}

\subsection{The ATLAS Liquid Argon Calorimeter System}

The ATLAS calorimeter system, shown in Figure 3.1, can be divided into the tile and liquid argon calorimeter systems which, though differing in their design, are both responsible for energy measurement. One can note in the figure three distinct regions corresponding to the liquid argon system: the two end-cap components and a central barrel region. The two end-cap regions cover the pseudorapidity range $1.4<|\eta|<4.9$, and the electromagnetic barrel region covers the region for which $|\eta|<1.5$ [4]. Each of these three regions is housed in its own cryostat filled with liquid argon which acts as the active medium for these calorimeters. 


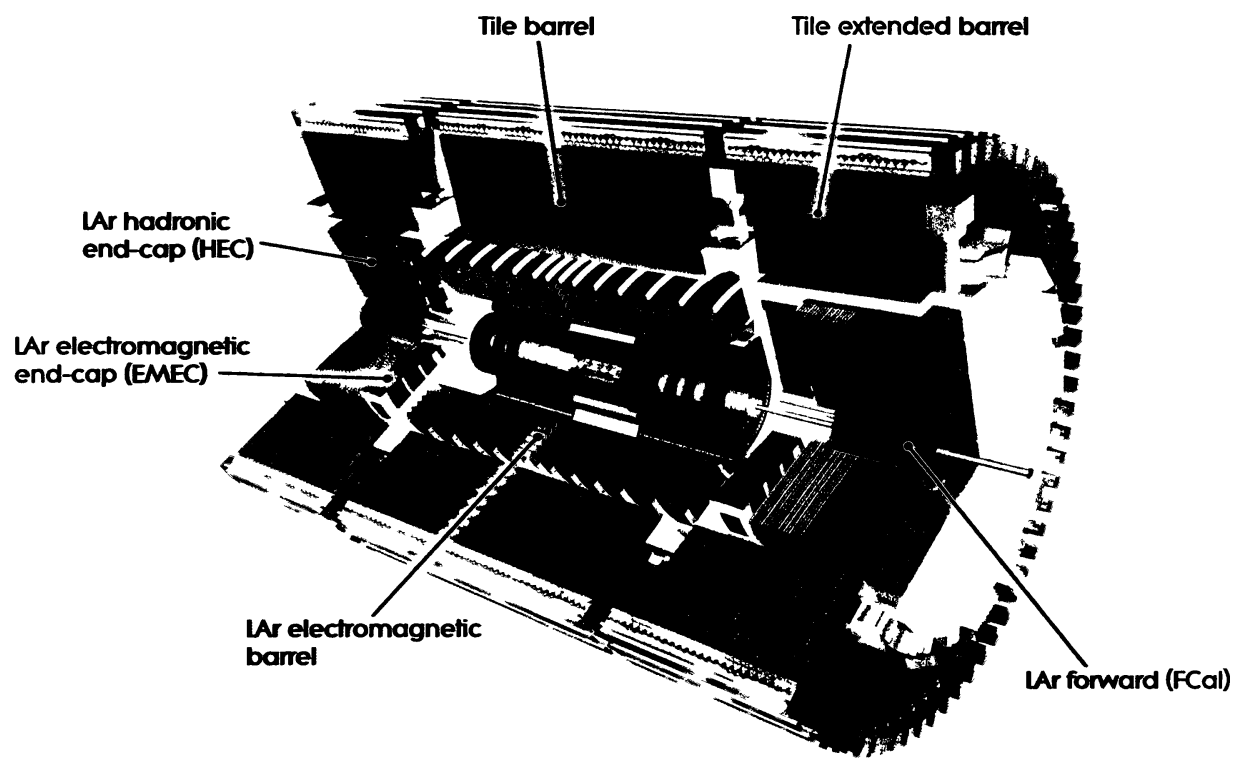

Figure 3.1: A cut-away view of the ATLAS calorimeter system [2].

\subsection{The ATLAS Forward Calorimeters}

The end-cap calorimeters which cover the range $1.4<|\eta|<4.9$ can in fact be subdivided once more into the electromagnetic and hadronic end-caps for $|\eta|<3.2$ and the forward calorimeters which overlap the end-cap $\eta$-range and cover from $3.1<|\eta|<4.9$, although all lie within the same cryostat as previously mentioned.

The two ATLAS Forward Calorimeters, which are the focus of the current analysis, are situated at a distance of approximately $4.7 \mathrm{~m}$ on either side of the central interaction point, bordering the LHC beam pipe. They were designed to measure the energy of 
particles in the high- $\eta$ region and, based on their placement, to reduce the background radiation in the muon spectrometer.

\subsubsection{Design and Construction}

Each FCal consists of three modules, referred to as FCal 1, FCal 2, and FCal 3, which have a depth of $45 \mathrm{~cm}$ and differ slightly in their design. All three modules together provide a depth of approximately 10 nuclear interaction lengths ${ }^{1}$. Generally the first module, FCal 1, is referred to as an electromagnetic calorimeter, with the remaining modules referred to as hadronic calorimeters. The absorbing material for FCal 1 is primarily copper, whereas that for FCal 2 and 3 is tungsten. Figure 3.2 shows a scaled depiction of the upper portion of one of the ATLAS Forward Calorimeters. The copper alloy plug or shielding which can be seen in this figure, though not strictly a part of the forward calorimeter system, acts to further reduce the radiation which would result in background in the muon spectrometer system.

The FCal modules consist of a metal matrix containing a large number of evenly spaced electrodes which provide the FCal units with very fine lateral segmentation. The electrodes themselves lie parallel to the LHC beam pipe and are made up of co-axial rods and tubes. In the case of FCal 1, both the rods and tubes are made of copper, whereas the rods for the other modules are made of tungsten. Furthermore, in FCal 2 and FCal 3, tungsten slugs are arranged symmetrically around each of the copper tubes in order to provide more absorbing material, whereas in FCal 1 additional copper material is used to support the rods and tubes rather than tungsten slugs.

\footnotetext{
${ }^{1}$ Refer to Section 2.5 on the properties of hadronic showers
} 


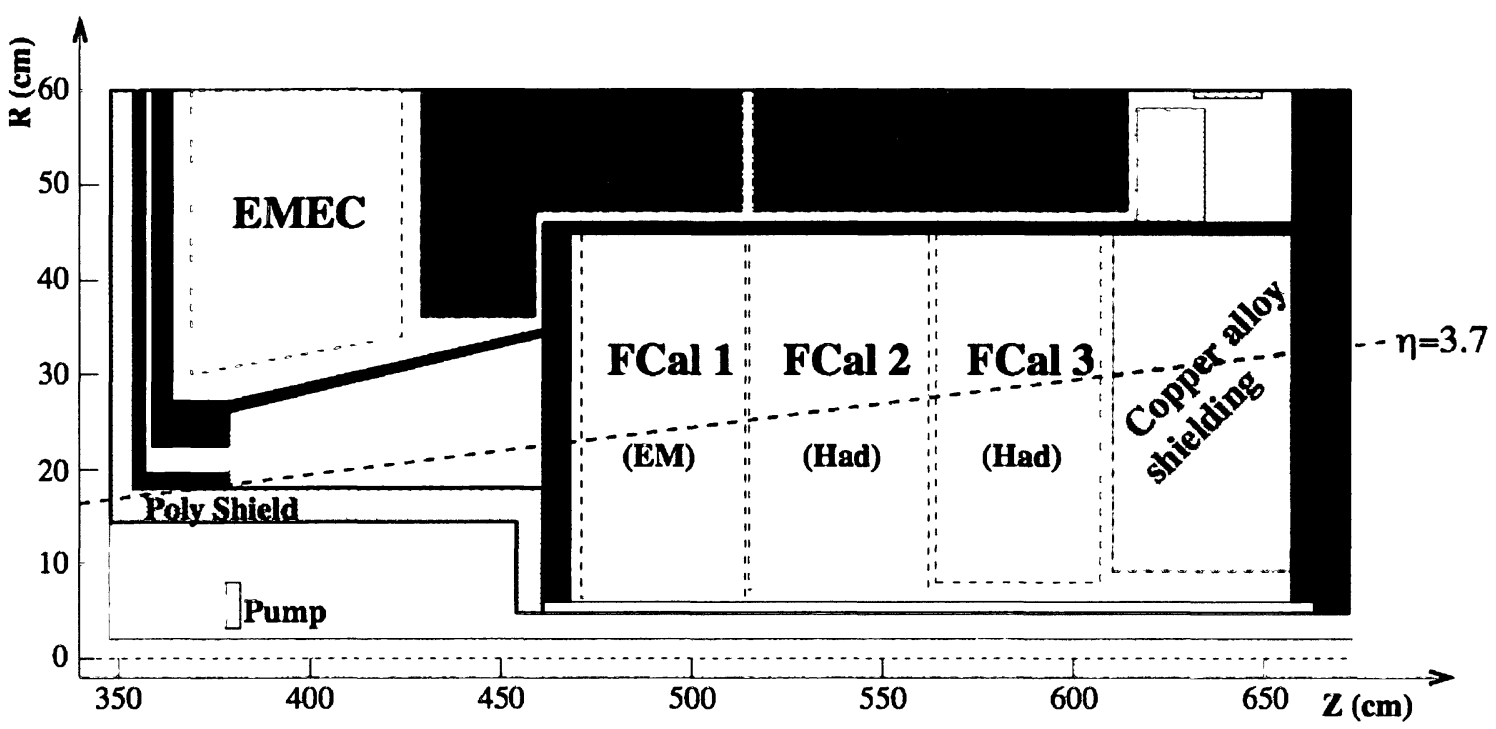

Figure 3.2: The upper portion of the FCal modules shown within the rest of the ATLAS liquid argon calorimeter system. Also shown is the path that would be taken by a particle travelling at a pseudorapidity value of $\eta=3.7$.

Liquid argon, the sampling calorimeter's active medium, fills the gap between the

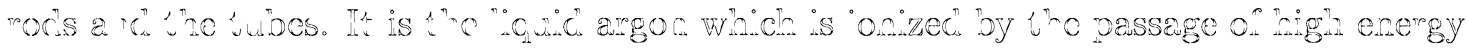
particles, producing an ionization pulse which is read out by the detector's electronics. Such a thin gap of liquid argon is required due to the high level of particle flux present in this region of the ATLAS detector, specifically to reduce the drift time of the ionization electrons. The gap itself is maintained by a thin PEEK fibre, helically wound around the inner rod. The radius of the fibres can be seen in Table 3.1, which also shows the depth of each FCal module in radiation lengths and nuclear interaction lengths, along with other design parameters of interest. 
The high level of flux additionally requires that one closely monitor the temperature of the liquid argon, due to the narrow temperature range associated with its liquid state. Liquid argon was specifically chosen for the active medium as it is a relatively inexpensive and radiation hard material.

Table 3.1: Selected design values of interest for the FCal modules. The values are taken from a similar table in [4].

\begin{tabular}{|c|c|c|c|}
\hline & FCal 1 & FCal 2 & FCal 3 \\
\hline \hline Mass $[\mathrm{kg}]$ & 2119 & 3826 & 3695 \\
\hline Main absorber material & Copper & Tungsten & Tungsten \\
\hline LAr gap width [mm] & 0.269 & 0.376 & 0.508 \\
\hline Depth in radiation lengths $\left(X_{0}\right)$ & 27.6 & 91.3 & 89.2 \\
\hline Depth in nuclear interaction lengths $\left(\lambda_{I}\right)$ & 2.66 & 3.68 & 3.60 \\
\hline Number of readout channels & 1008 & 500 & 254 \\
\hline
\end{tabular}

Figure 3.3(a) shows a rendition of a typical FCal electrode, where one can note the PEEK fibre wound around the central rod. The FCal electrodes are electrically connected to form a tube group, which are then summed to form readout channels. The terms cell and readout channel will be used interchangeably to denote the finest level of lateral readout granularity available for the FCal modules. Readout channels are typically composed of sixteen individual electrodes in FCal 1, though this is not always the case. Smaller groups are used particularly in the higher- $\eta$ range closer to the beam pipe which result in a smaller effective volume for some readout channels. The electronic readouts for FCal 2 and FCal 3 are similar in design to those of FCal 1, with the main difference that 24 


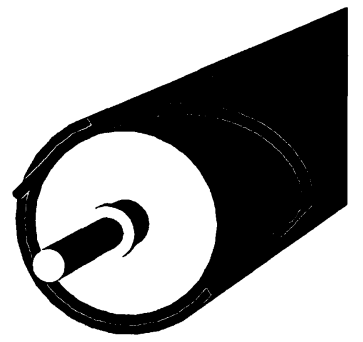

(a) A depiction of a typical FCal electrode consisting of a central rod surrounded by a copper tube. The gap between the two, filled with liquid argon, is held constant by means of a helically wound PEEK fibre.

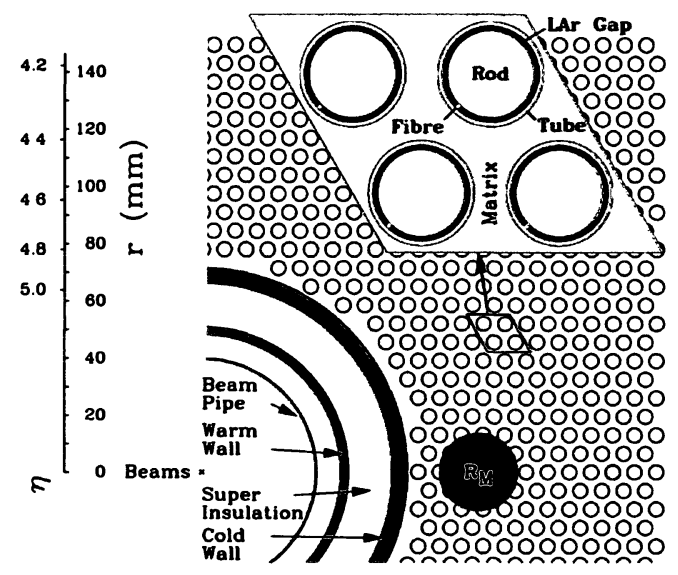

(b) The forward calorimeter cell structure is shown within the copper matrix. The shaded circular region shows a circle with one Molière radius representative of the scale of lateral electromagnetic shower development. The enlarged diamond-shaped region representing a collection of four individual electrodes is also highlighted.

Figure 3.3: Artistic renditions of a) a typical FCal electrode and b) a collection of electrodes as depicted on a view of the front surface of a forward calorimeter module.

electrodes are read out together for a single cell in FCal 2, and 36 in FCal 3.

The electronic signals themselves from each of the readout channels result from the details of the collection of the ionization in the liquid argon gaps. A typical pulse after shaping for each of the FCal modules is shown in Figure 3.4. The figure is from a 2008 publication in which a more descriptive layout of the ATLAS forward calorimeters is presented [12]. Black sampling points are shown at $25 \mathrm{~ns}$ intervals which is the same as the time between the design LHC proton bunch crossings. The raw ionization drift signal which is not shown, is triangular in form with the peak of the signal pulse close to its highest point. The amplitude of the shaping pulse is proportional to the peak of the 
raw signal.

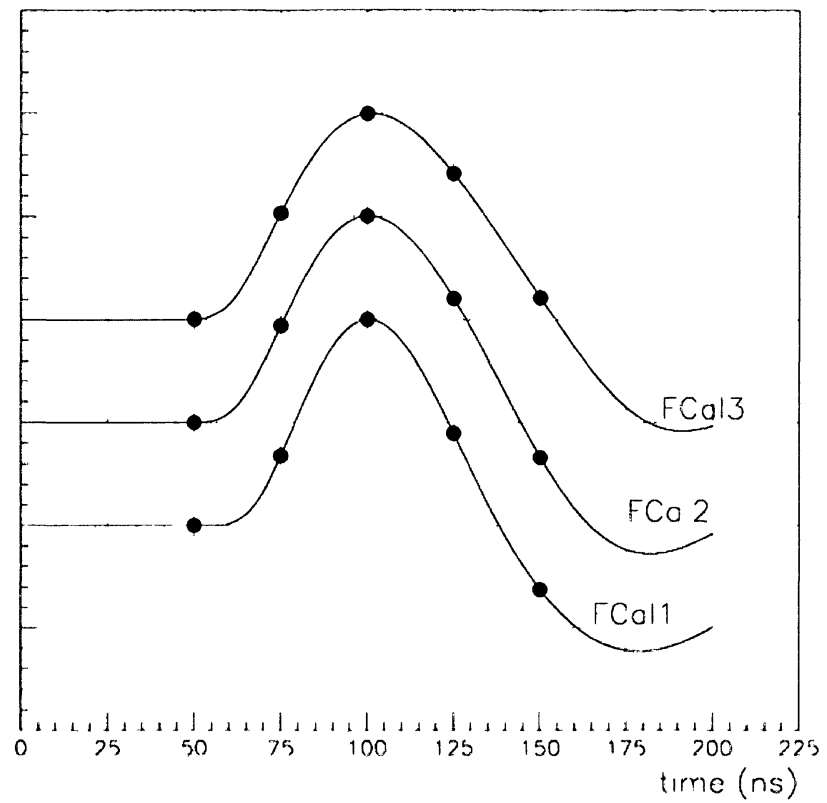

Figure 3.4: Sample liquid argon pulse shapes, shown with sampling points at every 25 ns interval with arbitrary units selected for the vertical axis. The peak of the pulse corresponds to the energy measurement for a given readout channel. The shapes of the pulses vary slightly between the FCal modules, though the vertical spacing visible between and position of the shapes is strictly for ease of comparison. The figure is taken from [12].

An optimal filtering technique [13] is used to determine the peak of the pulse which is a measure of the energy deposited in the channel in units of $A D C$ counts. Section 5.3 briefly discusses the conversion which is made from $A D C$ counts to units of $\mathrm{GeV}$, though it is recognized that several details have been omitted with regards to the electronics and signals involved; such details are expanded upon in [13] and [14]. In summary it can 
be seen that one is able to go from an initial ionization drift signal to a pulse shape for which the peak provides an energy measurement for the particular readout channel. This energy measurement will be referred to in Chapter 5 in determining the reconstructed energy for a given test beam particle.

\subsubsection{Commissioning and Ongoing Work}

The ATLAS forward calorimeters are currently situated nearly $100 \mathrm{~m}$ underground, housed in liquid argon cryostats and surrounded by the other components of the giant ATLAS detector. Here they continue, at the time of writing, to be used to measure the energy of high-energy particles resulting from collisions at the detector's centre. Although the commissioning of the forward calorimeters has taken place, as with the other components of the detector, it is necessary for ongoing work to be done in order to best interpret the signals produced in the calorimeter.

The measurement of significant, unanticipated amounts of transverse energy is one of the signatures of the presence of physics beyond the standard model, and the measurement of jet energies is necessary in order to identify many key physics processes. The accuracy and precision of both of these measurements relies on successful energy measurements with the calorimeter system. The data taken at the 2003 FCal test beam continue to be analyzed to optimize the calibration of the FCal. The following chapter summarizes the setup of this test beam. 


\section{Chapter 4}

\section{FCal Test Beam}

\subsection{Test Beam Setup and Overview}

The summer and fall of 2003 saw the collection of data for the 2003 forward calorimeter test beam, carried out at CERN using beams of particles originating from CERN's H6 beamline. One of two primary aims of the 2003 test beam was to calibrate and determine both the energy response and resolution of one of the FCal units, by subjecting it to beams of particles of known energy and analyzing the detector response. Beams of both electrons and pions were used separately at various discrete energy values in order to determine the calorimeter's intrinsic response at the electromagnetic scale, and to investigate its response to hadronic particles, respectively. The other primary aim of the FCal test beam was to understand and quantify the energy response at the inner edge of the FCal which will be explained in Chapter 10 .

During the collection of data at CERN, the FCal modules were placed in a $1.5 \mathrm{~mm}$ 
thick stainless steel 'bathtub' which was housed in a liquid argon cryostat, as shown in Figure 4.1, to simulate the conditions that will be present during actual data taking. In the real ATLAS configuration, particles interacting with the FCal would typically originate near the interaction point at the centre of the ATLAS detector, and would not travel towards the FCal in a path perpendicular to FCal1's front face. As such, the modules were set at an angle of approximately three degrees relative to the incoming beams of particles, as can be crudely seen in the figure. The impact angle could be adjusted based on the translation and rotation of the cryostat. More specifically, different beam impact points were selected as will be summarized below, and the impact angles for these points were chosen in order that the test beam particles always appeared to have originated where the centre of the ATLAS detector would be situated in the ATLAS coordinate system.

\subsection{Instrumentation During Data Collection}

It is important to highlight a few other key components of the test beam setup as shown in Figure 4.1. It should be pointed out that a publication of a previous analysis of the data from the 2003 FCal test beam will be referred to for a number of values and design specifications in the following subsections, and will be cited here rather than being repeated throughout the text [13]. 


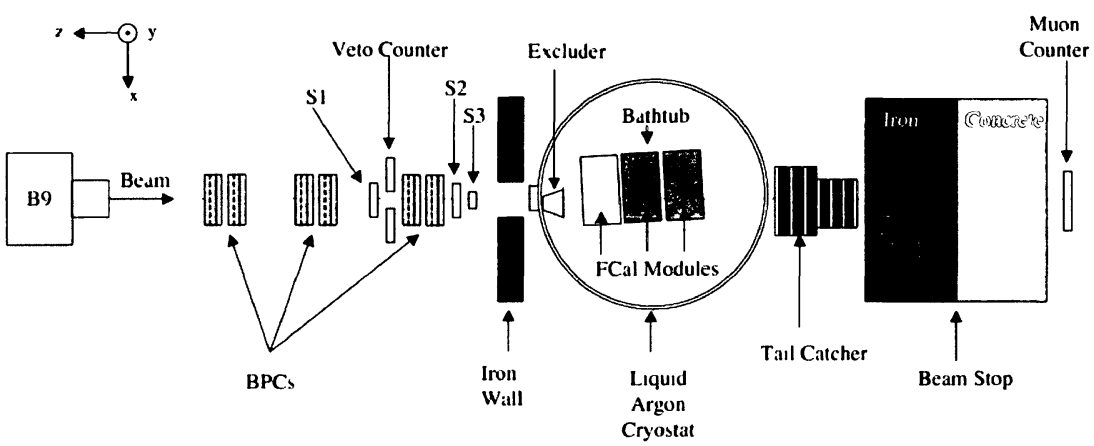

Figure 4.1: The setup for the 2003 FCal test beam. Shown also is the ATLAS coordinate system in which the positive $\mathrm{x}$-direction would point towards the centre of the LHC ring, and in which the positive y-direction, shown here to be out of the page, would point vertically upwards away from the centre of the Earth.

\subsubsection{BPC Tracking Information and Beam Envelope Cuts}

The Beam Positioning Chambers (BPCs), consisting of multi-wire proportional chambers, were situated upstream from the bathtub containing the liquid argon and FCal modules. The BPCs were used only in the test beam configuration and the information they provided will not be available during the collection of actual data during collisions within the ATLAS detector. Their purpose was to provide positioning information for beam particles in order to calculate the $x-y$ position at which a given particle struck the front face of FCal 1. A projected impact point could also be calculated for the front faces of the remaining two FCal modules. This positioning information was subsequently used to determine which cells to include in one of the clustering algorithms which will be described in the following chapter. The BPCs were situated in three main sets, as can be 
seen in Fig. 4.1. Though all devices were similar in operation, the spatial resolution for both the first and third sets was approximately $150-200 \mu \mathrm{m}$, as compared to a slightly poorer spatial resolution of approximately $300 \mu \mathrm{m}$ for the middle set.

In addition to providing an impact position on the surface of FCal 1 for each particle, the tracking information from the BPCs and the information from the scintıllatıon trigger and veto counters [12] on either side of the BPCs also allowed for the removal of multiple particles and those events corresponding to beam particles which scattered upstream of the FCal modules. These are referred to as beam profile or beam envelope cuts. The average beam profiles for electrons and hadrons are different which allows the possibility to partially differentiate these types of particles.

\subsubsection{Tail Catcher, Muon Counter and Beam Stop}

It was advantageous to be able to identify the signals resulting from muons which accounted for a small fraction of the particles present within the beams. A series of runs were taken with muon beams to investigate the FCal response to these particles, though such identıfication was not the goal of the present analysıs. The muon counter situated at the furthest point downstream in the test beam configuration was often used in conjunction with coincidence signals from the tanl catcher [12]. These detectors take advantage of the fact that whereas muons deposit relatively little energy in the form of ionization electrons as they traverse the experimental setup and ultimately produce a signal in the tail catcher, electrons deposit all of their energy in the forward calorimeter, predominantly in the form of electromagnetic cascades or showers ${ }^{1}$, before reaching the rear face

\footnotetext{
${ }^{1}$ Refer to Section 24 on the properties of electromagnetic showers
} 
of FCal3. A charged particle which leaves a signal in the muon counter can therefore be identified as a candidate muon. An iron and concrete beam stop was situated between the tail catcher and muon counter, as can be seen in Figure 4.1 as a means to further restrict particles other than muons from reaching the muon counter. In practise however, muons can also be created as a result of hadronic shower, and making a cut on events for which a signal is recorded in the muon counter in an effort to reduce muon contamination would introduce a bias. The tail catcher and muon counter are not used in the case of the present analysis, and their description here is included merely for completeness.

\subsection{3 Čerenkov Detector and CEDAR Cuts}

One means of removing the signals from unwanted beam particles is by making use of a gas differential Čerenkov detector [12] referred to as a CEDAR $^{2}$ detector [15]. Such devices were used during the collection of test beam data in order to distinguish between electrons and pions in electron beams, and between pions and protons in pion beams. The CEDAR detectors were optimized to distinguish between such particles at nominal beam energies below $100 \mathrm{GeV}$. The removal of protons in pion data runs was made possible by the information from the CEDAR detectors at all nominal beam energy values except at $200 \mathrm{GeV}$, where such information was not available. Throughout this paper, the removal of events in this way will be referred to as CEDAR cuts.

\footnotetext{
${ }^{2}$ ČErenkov Differential Counter with Achromatic Ring Focus
} 


\subsubsection{Summary of Standard Data Cuts}

In addition to the cuts mentioned above, more sophisticated beam cleaning cuts were also included which further reduced the number of events for which multiple particles are recorded or, similarly to the beam envelope cuts, for which the particle of interest scattered upstream of the calorimeter. Beam cleaning and envelope cuts of this type have also been used in other similar experiments [14] [16].

The standard cuts referred to in the present analysis are therefore meant to include the beam envelope cuts, beam cleaning cuts and, for certain energies as explained above, the $C E D A R$ cuts. Such standard cuts are similar to those used for other analyses of FCal test beam data [17]. Neither the determination of which events passed the cuts, nor a systematic study as to whether such cuts could be improved were carried out in the present analysis, though systematic studies were carried out by other test beam collaborators for this purpose.

\subsection{Beam Impact Points}

Five distinct impact points were chosen for both the electron and pion beams, as can be seen in Fig. 4.2. The beams themselves had a diameter of approximately $5 \mathrm{~cm}$, and could be directed at each of the impact points by sliding and rotating the cryostat in the horizontal plane, or by adjusting the current in the final bend magnet B9 to adjust the impact position in the vertical plane ${ }^{3}$.

The two outer points referred to as $4 \mathrm{~L}$ and $4 \mathrm{H}$ were chosen as showers resulting from

\footnotetext{
${ }^{3}$ Refer to Fig. 4.1
} 


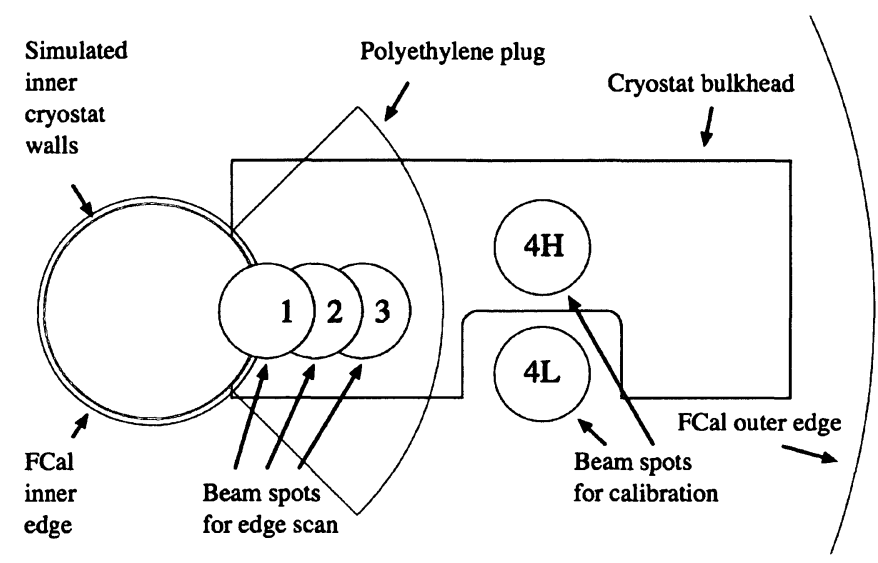

Figure 4.2: The five points selected for the 2003 test beam, including the two outer points $(4 \mathrm{H} \& 4 \mathrm{~L})$ and three inner points $(1,2 \& 3)$. Shown is the position of the impact points on the front face of FCall.

electrons and pions at these positions would be fully contained. Beam energies ranging from 10 to $200 \mathrm{GeV}$ were used at these two points. The so-called inner points are located closer to the beam pipe and correspond to higher pseudorapidity ${ }^{4}$ values in the ATLAS coordinate system. The inner points are labelled as 1, 2 and 3 as shown in Figure 4.2. One cannot expect full shower containment for these points due to their proximity to the edge of the calorimeter. The near vacuum within the beam pipe will allow shower constituents to spray into and across the beam pipe, resulting in energy deposits on the opposite side of the FCal modules and a lower response in terms of the reconstructed energy for a given event. This will be made clearer in Chapter 5. Indeed, the positions of these inner points were selected to investigate just this effect. Many physics analyses within the ATLAS

\footnotetext{
${ }^{4}$ As defined in Equation (1.1)
} 
collaboration will involve high- $\eta$ jets and particles, and a good understanding of shower development close to the beam pipe will be required in order to accurately and precisely measure the energy of such jets and particles. Table 4.1 shows the position of each of the beam impact point positions both in terms the polar angle $\theta$ and pseudorapidity ${ }^{5} \eta$.

Table 4.1: Values of the dimensionless pseudorapidity, $\eta$, of the centre of each beam impact point on the face of FCal1 and its polar angle, $\theta$, as measured from the centre of the LHC beam pipe.

\begin{tabular}{|c|c|c|}
\hline Impact Point & $\theta$ & $|\eta|$ \\
\hline \hline 1 & $0.88^{\circ}$ & 4.9 \\
\hline 2 & $1.25^{\circ}$ & 4.5 \\
\hline 3 & $1.61^{\circ}$ & 4.3 \\
\hline $4 \mathrm{H}, 4 \mathrm{~L}$ & $2.98^{\circ}$ & 3.7 \\
\hline
\end{tabular}

Though it would be desirable to have had a full scan of energy values at the inner points as well as the outer points, only $200 \mathrm{GeV}$ data is available for the former. As such, good agreement will be required between studies of simulated Monte Carlo data and test beam data for the case of $200 \mathrm{GeV}$ pions at these inner points before one can put faith in the results of Monte Carlo analyses at other beam energy values. At the time of writing, such comparisons between Monte Carlo and data, though not the subject of the present analysis, are being carried out by other members of the Toronto and Carleton ATLAS FCal test beam groups. These comparisons will be very important for the development of an accurate Monte Carlo simulation package at different energies for the FCal system.

\footnotetext{
${ }^{5}$ Refer to Section 1.4 .1
} 


\subsubsection{Modelling of Upstream Material}

Initially one might expect a similar response from beams of particles directed at the two outer impact points $4 \mathrm{H}$ and $4 \mathrm{~L}$, shown in Fig. 4.2. Upon closer inspection however, one will note the addition of extra material labelled as the cryostat bulkhead which appears over impact point $4 \mathrm{H}$, as well as the three inner points. This was just one of the ways in which an attempt was made to simulate as much as possible the conditions under which the FCal will collect data in the real ATLAS environment. Particles travelling towards the FCal modules from the interaction point at the centre of the ATLAS detector will first have to traverse the metal cryostat bulkhead prior to reaching the FCal system. This cryostat bulkhead was modelled by fastening a $5.0 \mathrm{~cm}$ slab of aluminum to the inside wall of the bathtub containing the FCal modules. An analysis of data from the $4 \mathrm{~L}$ position provides the intrinsic response one can expect from the FCal with no upstream material. This can then be contrasted with the response one obtains with inclusion of upstream material that will be present with real ATLAS data.

In addition to the material added to model the cryostat bulkhead, an amount of polyethylene was placed in front of the test beam's path upstream of the cryostat to simulate the tube portion of the ATLAS JM moderator, as well as an additional piece of polyethylene in front of the three inner points to model a plug of moderator material that would be present upstream of those points. The latter was fastened to the outside of the front face of the bathtub wall. Finally, the position of an ion pump upstream of the innermost point, position 1, was modelled by means of an additional $30 \mathrm{~mm}$ thick piece of aluminum which was placed approximately $900 \mathrm{~mm}$ upstream of the FCal modules. 


\subsection{Summary of Pion and Electron Data}

Table 4.2 shows the total number of pion triggers from all good pion runs before the application of any cuts. The values in the table are in thousands of events, and are shown for each beam energy value both for $4 \mathrm{H}$ and $4 \mathrm{~L}$. Table 4.3 similarly shows the pion triggers for the inner beam impact points 1,2 , and 3, for which only $200 \mathrm{GeV}$ data are available.

Table 4.2: Total number of triggers (in thousands) for all good pion runs prior to any cuts, shown for each beam energy value at the two outer beam impact points. Note that there were no good $20 \mathrm{GeV}$ runs taken for point $4 \mathrm{~L}$.

\begin{tabular}{|c||c|c|c|c|c|c|c|c|c|}
\hline \multicolumn{1}{|c||}{$\begin{array}{c}\pi \text { Triggers } \\
\text { Before Cuts }\end{array}$} & \multicolumn{8}{|c|}{ Nominal Beam Energy (GeV) } \\
\cline { 2 - 10 } & 10 & 20 & 40 & 60 & 80 & 100 & 120 & 150 & 200 \\
\hline \hline $4 \mathrm{H}$ & 960 & 408 & 780 & 816 & 660 & 444 & 612 & 629 & 648 \\
\hline $4 \mathrm{~L}$ & 1086 & $\mathrm{n} / \mathrm{a}$ & 900 & 408 & 816 & 414 & 612 & 612 & 672 \\
\hline
\end{tabular}

Table 4.3: Total number of triggers (in thousands) for all good $200 \mathrm{GeV}$ pion runs prior to any cuts, shown for the three inner beam impact points.

\begin{tabular}{|c||c|}
\hline $\begin{array}{c}\pi \text { Triggers } \\
\text { Before Cuts }\end{array}$ & Nominal Beam Energy \\
\cline { 2 - 2 } & $200 \mathrm{GeV}$ \\
\hline \hline 1 & 660 \\
\hline 2 & 432 \\
\hline 3 & 624 \\
\hline
\end{tabular}




\subsection{Noise Treatment}

During the collection of test beam data, in addition to the number of pion or physics triggers summarized in Tables 4.2 and 4.3, a number of randomly triggered events were taken for each readout channel within each run. The particular readout channel's pedestal value was subtracted from each of these measurements, resulting in a Gaussian distribution in ADC counts centred roughly at zero. The standard deviation of such distributions is what is typically referred to as the noise for a particular channel. Certain sets of runs and particular channels were known to be more noisy than others [13].

Having selected one of the randomly triggered events from a uniform distribution, the particular value associated with each readout channel for that random event was associated with a physics event. Summing over all of the readout channels included for a particular physics event, which will be made clearer in the following chapter, each physics event has then associated with it an energy measurement both from a real physics event and from a randomly triggered event. The energy values associated with the random trigger events are summed over the included readout channels. This is repeated for all events such that a distribution is obtained, and it is the standard deviation of this distribution which is later subtracted in quadrature from the energy resolution to obtain the noise-subtracted energy resolution, which will be introduced in Section 5.2. 


\subsection{Offline Test Beam Data Analysis}

The 2003 FCal Test Beam data were analyzed using Athena, the standard software package used by the ATLAS collaboration, specifically version 13.0.40. The timing, clustering of cells, event reconstruction and noise treatment were all handled using this program. The Athena output is in the form of a file containing a large number of arrays of event- and run-specific variables, including but not limited to the energy deposited in each cell for a given event. The contents of this file can be analyzed by making use of ROOT, a object oriented data analysis and histogramming package based on the $\mathrm{C} / \mathrm{C}++$ programming language [18]. The code written for the present analysis employed packages from the ROOT libraries. 


\section{Chapter 5}

\section{Reconstructing Energy in the}

\section{Forward Calorimeter}

The purpose of this chapter is to describe the calculations made in order to arrive at an energy measurement based on the signals provided by the ATLAS forward calorimeters, particularly for the case of the 2003 test beam data summarized in the preceding chapter. It is recognized that a number of steps were necessary to go from the measured ionization pulse to a final energy measurement in units of either ADC counts or GeV for a given readout channel in the forward calorimeter. In the interest of brevity, only the most important steps will be described in full detail; additional details can be found in the technical report outlining the design of the ATLAS forward calorimeters [12] and the results of previous analyses of the 2003 test beam data [13].

For the purposes of the present thesis, the term reconstructed energy refers to the 
energy measurement returned by the forward calorimeter for a specific particle ${ }^{1}$ - in this case a pion or an electron. It should be recognized, given the nature of such particles' interactions with the calorimeter modules, that the reconstructed energy for one individual particle will be a summation of the energy deposited for its shower constituents.

\subsection{Summing Energy in the FCal Modules}

\subsubsection{Inclusion of Active Readout Channels}

A pion will deposit its energy, in the form of a hadronic shower, throughout the volume of the three forward calorimeter modules. Provided the shower is fully contained - a

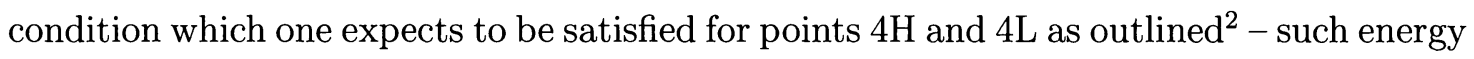
deposits will be distributed amongst the readout channels present in the calorimeter system.

During the analysis of test beam data not all FCal readout channels were included; Figure 5.1 shows the regions of each of the three modules containing active readout channels, chosen to match the locations of the beam impact points.

Letting $E_{\imath}^{\mathrm{EM}}$ represent the sum of electromagnetic scale energy based on all included readout channels in the $i^{\text {th }}$ FCal module, the reconstructed energy at the electromagnetic scale for the particle or event of interest is given by:

\footnotetext{
${ }^{1}$ The term reconstructed energy will also be used to describe a quantity based on a distribution of events, so there is the potential for ambiguity. This will be made more clear in Section 5.1.3

${ }^{2}$ Refer to Section 4.3
} 


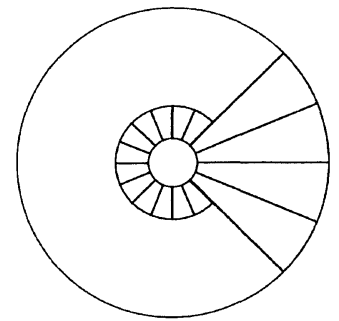

FCal1C

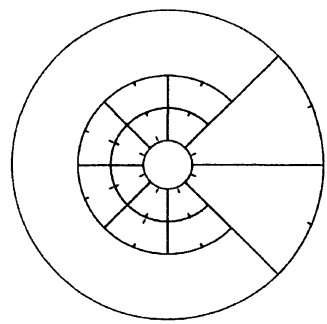

FCal2C

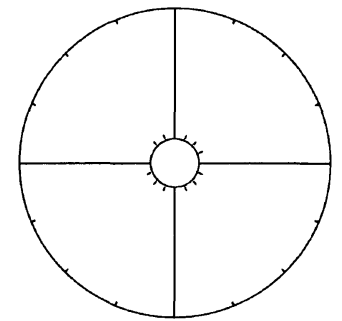

FCal3C

Figure 5.1: Representation of regions of the FCal modules which had active readout channels during the collection of data for the FCal test beam.

$$
E_{\mathrm{Reco}}^{\mathrm{EM}}=\sum_{\imath} E_{\imath}^{\mathrm{EM}}=E_{1}^{\mathrm{EM}}+E_{2}^{\mathrm{EM}}+E_{3}^{\mathrm{EM}}
$$

Recall that $E_{\text {Reco }}^{\mathrm{EM}}$ should ideally represent the accurate energy of a test beam electron, whereas in the case of a test beam pion, it will be below the nominal beam energy due to the nature of hadronic showers, as discussed in Section 2.7.

\subsubsection{Cell Clustering Algorithms}

The inclusion of cells in which little or no energy has been deposited will do little more than broaden the distribution of reconstructed energies based on the cumulative sum over the FCal modules in Equation (5.1). The cause of such broadenıng is due to the random fluctuations or noise in such cells far from the shower deposits. Such noise is present in all cells of course, though in the case of cells with a significant energy deposit and in a region of interest, it is an inescapable requirement that both the signal and noise be read 
out together. The treatment of noise was discussed briefly in Section 4.5, and additional details are available in previous ATLAS FCal test beam publications [13] [12].

The principal point to be made is that, in the interest of reducing unnecessary noise, it is preferable to exclude, when possible, those cells with a negligible energy deposit. Two common algorithms are employed for this purpose and will be regularly referred to throughout the thesis. Both of these algorithms are said to serve the purpose of noise reduction for the reasons outlined above.

Cylindrical clustering is an algorithm well suited to the specific design of the forward calorimeter system. The BPC tracking information ${ }^{3}$ for a given particle is used to determine the particle's projected impact point on the front surface of each FCal module. Given the small but non-negligible incidence angle for test beam particles ${ }^{4}$, it should be recognized that this projected impact point will be situated at different $x$ and $y$ coordinates for the three modules. For the $i^{\text {th }}$ module, only those cells whose centroid falls within a $16 \mathrm{~cm}$ distance from the impact point of that module are included in the overall sum to determine the value of $E_{i}$ in Equation (5.1). Previous analyses by other FCal test beam group members determined $16 \mathrm{~cm}$ to be the optimized distance for the cylindrical clustering algorithm in the case of pions ${ }^{5}$.

\footnotetext{
${ }^{3}$ Refer to Section 4.2.1

${ }^{4}$ Refer to Section 4.1

${ }^{5}$ For the case of electron data, $8 \mathrm{~cm}$ cylindrical clustering was used
} 
Topological clustering is an algorithm developed by members of the ATLAS collaboration, and is important in the implementation of the Local Hadronic Calibration [19] [20] package currently used in the analysis of ATLAS data. The Local Hadronic Calibration algorithm involves the classification, either electromagnetic or hadronic, of a particular shower in the ATLAS calorimeters based on several of its properties. This is done in order to determine if any hadronic weights need to be applied. It continues to be an important topic insofar as the reconstruction of a jet of several particles is comprised of a combination of showers which are either electromagnetic or hadronic ${ }^{6}$ and ongoing work is done at the time of writing in order to optimize the package to best make use of the data from the ATLAS calorimeters.

The topological clustering algorithm itself first designates as a seed any cell for which the measured energy deposit is above a certain threshold; this threshold is set by default to be four times the noise ${ }^{7}$. Additional neighbour cells adjacent to this seed cell are then merged with the original seed, though these cells are only included provided they too have an energy deposit exceeding a second threshold; the default value is now twice the noise. The iteration of this second step then continues until such time as no more cells are added, and in this way a 'cluster' begins to take shape. At the third and final step, all cells situated on the periphery of the neighbour cells are also included; a third threshold exists for the inclusion of these final cells, though by default the threshold has no lower bound, so all bordering cells are included.

\footnotetext{
${ }^{6}$ Though a significant fraction of hadronic showers themselves are electromagnetic as previously mentioned.

${ }^{7}$ The noise in this case is not the same as that defined in Section 4.5 since it requires channel-specific values. The optimal filtering technique mentioned in 3.2.1 is used for this purpose.
} 


\begin{tabular}{|c|c|c|c|}
\hline \multirow{2}{*}{\multicolumn{4}{|c|}{ 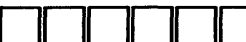 }} \\
\hline & & & \\
\hline 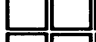 & & & \\
\hline & & & \\
\hline & & & \\
\hline & & & \\
\hline
\end{tabular}

Figure 5.2: A visualization of the topological clustering algorithm, in which cells are only included for a particular event if they yield a certain threshold energy with respect to the noise. Seed (red), neighbour (orange), and periphery (yellow) cells are shown, as well as the clear cells which do not pass the requirement for inclusion into the cluster.

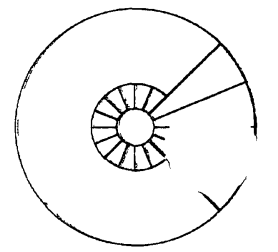

FCal1C

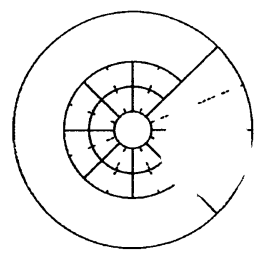

FCal2C

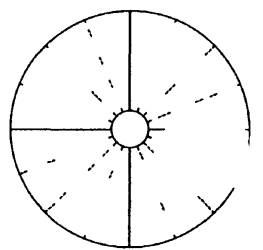

FCal3C

(a) A representation of the cylindrical clustering algorithm, in which all cells are included which are located within a $16 \mathrm{~cm}$ radial distance from the projected beam impact point for each module

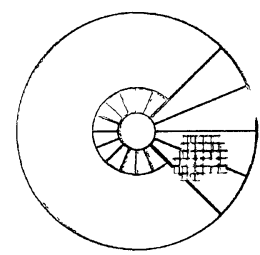

FCal1C

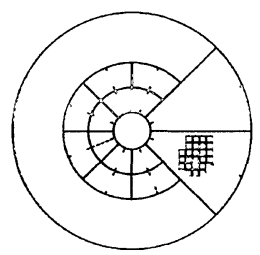

FCal2C

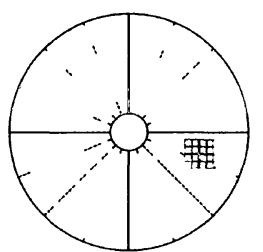

FCal3C

(b) A representation of the topological clusterıng algorithm, in which cells are only included for a particular event if they meet a certain threshold energy with respect to the noise Cells are shown for illustratıve purposes only, and do not reflect their actual sizes in relation to the size of the modules

Figure 53 Two common clustering algorithms used throughout the FCal Test Beam analysis· a) cylindrical clustering and b) topological clustering. 
Figure 5.2 offers a visualization the topological clustering algorithm based on square cells. Though illustratively shown based on a grid, the topological 'neighbouring' can occur in three dimensions. A seed cell situated in FCal 1, for example, will look not only to its neighbouring cells in FCal 1, but also in FCal 2 when attempting to form a topological cluster.

Figure 5.3 illustrates the two cell clustering schemes used for the present analysis. It should be noted that the BPC tracking information is used for the cylindrical clustering algorithm, though it is not strictly required for the topological clustering. This fact is of importance in light of the fact that such tracking information was only available for the test beam setup and will not be available during the collection of ATLAS collision data. As such, although the cylindrical clustering algorithm seems well-suited for the forward calorimeters given their geometry of their design, it assumes, at least in its present state, a greater knowledge of tracking information than can be expected. Although the introduction of new clustering algorithms or combinations or modifications of the two presented is possible, it is also important to note that the test beam environment is an ideal one in the sense that it neglects the background and pileup which will be present in the high-luminosity environment during ATLAS collisions. Indeed, the energy pileup and overlapping events will result in $16 \mathrm{~cm}$ cylindrical clustering not being a viable clustering scheme in the ATLAS environment. One should remain cognizant of the limitations of each algorithm, and keep in mind when the tracking information is and is not used, when comparing the results in following chapters. 


\subsubsection{Double Gaussian Fit for the Reconstructed Energy}

One can apply Equation (5.1) in order to determine the reconstructed energy for an individual pion at the electromagnetic scale. This can be repeated with all pions within the test beam data for a given beam energy, and at a particular beam impact position. The distribution of all reconstructed energies will appear to be roughly Gaussian. Figure 5.4 shows an example of such a distribution. In this case the distribution of reconstructed energies is shown for all $200 \mathrm{GeV}$ pions events passing the standard cuts ${ }^{8}$. The mean of the distribution can be seen to be significantly lower than $200 \mathrm{GeV}$, as expected given the non-compensating nature of the calorimeter for hadrons.

An examination of the figure suggests the presence of an added shoulder above the mean peak not to be expected for a true Gaussian signal. This can be explained based on the design of the forward calorimeter modules. An incident pion will most likely initially strike copper - either the matrix or the rods of the electrodes. Another outcome, however, is that the pion strikes one of the small gaps of liquid argon between the anodes and cathodes. The relatively long nuclear interaction length ${ }^{9}$ of liquid argon compared with copper means that in this case the pion will tend to penetrate deeper into the calorimeter before initiating a hadronic shower, thereby resulting in a higher energy measurement than if it had simply struck one of the other materials. The relatively small area of liquid argon compared to absorber material results in this small but non-negligible high energy peak at the high-energy side of the distribution.

\footnotetext{
${ }^{8}$ The beam cleaning and beam envelope cuts summarized in Section 4.2. It should be reminded that the CEDAR cuts in this case were not applied, based on the information stated in Section 4.2.3, since the figure corresponds to $200 \mathrm{GeV}$ data.

${ }^{9}$ Refer to Section 2.5
} 


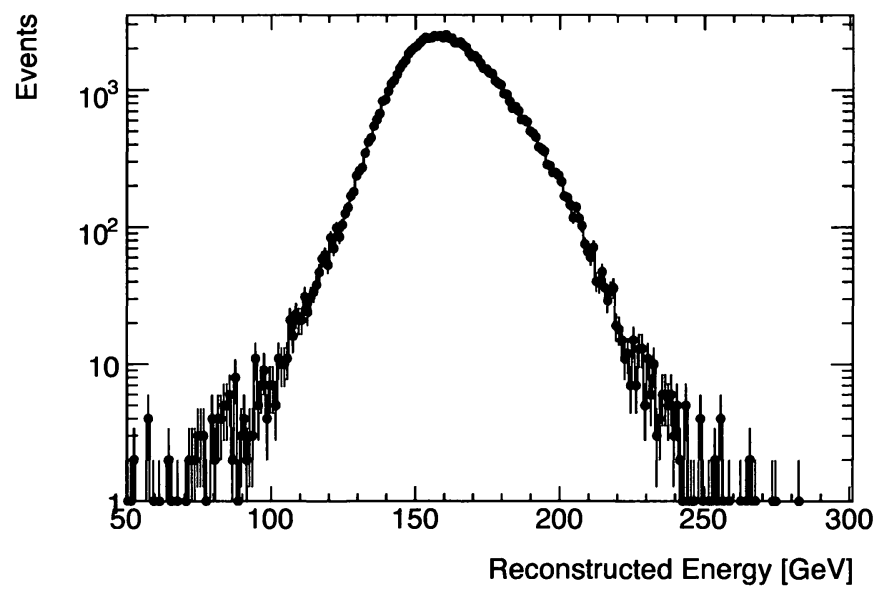

Figure 5.4: Sample EM scale reconstructed energy distribution for all $200 \mathrm{GeV}$ pion data runs at $4 \mathrm{~L}$.

To account for the shape of the distribution, a double Gaussian function is fitted to the data. In reality, the distribution is first fit to a single Gaussian, and the mean and variance values from this fit are then used to place loose constraints on the means of the two subsequent Gaussian functions. The fit utilizes a $\chi^{2}$ minimization algorithm and returns six parameter values with their errors. Equation (5.2) explicitly shows the double Gaussian function used, where the values $\left\{p_{\imath}\right\}$ correspond to the six parameters for the function. It is the first moment of this double Gaussian function which is then defined to be the reconstructed energy for a given beam energy.

$$
f(x)=p_{0} e^{\frac{-1}{2}\left(\frac{x-p_{1}}{p_{2}}\right)^{2}}+p_{3} e^{\frac{-1}{2}\left(\frac{x-p_{4}}{p_{5}}\right)^{2}}
$$

It should be pointed out that the term reconstructed energy, which previously referred 
to a particular particle, is now used to refer to the first moment of the double Gaussian fit, and it is in this latter sense that it will more often be used. The term mean reconstructed energy, although it would seem the natural choice as a substitution, is clearly not applicable in this case for the reasons outlined above. A distinction will be made in the case of any potential ambiguity.

\subsection{Energy resolution}

While it is important to reconstruct as accurately as possible the energy of the original particle in which one is interested, one would also like to have a measure of the spread in the distribution of reconstructed energies for beam particles. A standard measure of such a spread is what is known in calorimetry as the energy resolution, defined to be:

$$
\frac{\sigma_{\text {Reco }}}{E_{\text {Reco }}}
$$

where $\sigma_{\text {Reco }}$ is the second central moment of the reconstructed energy energy distribution summarized in the preceding section. The $E_{\text {Reco }}$ term in the denominator refers to the reconstructed energy - the first moment of the double Gaussian fit.

The energy resolution for a given calorimeter system is expected to vary with a particle's energy, such that a standard parameterization can be employed:

$$
\frac{\sigma_{\text {Reco }}}{E_{\text {Reco }}}=\frac{a}{\sqrt{E}} \oplus b \oplus \frac{c}{E}
$$

Here the terms $a, b$, and $c$ are referred to as the sampling or stochastic term, the constant 
term, and the noise term, respectively, and the term $\oplus$ denotes a sum in quadrature. The stochastic or sampling term, $a$, which tends to dominate [8], is affected by any processes governed by Poisson statistics. This includes fluctuations in the energy deposited in each sampling layer. The constant term, $b$, incorporates any calibration errors, manufacturing non-uniformities or other systematic effects with respect to energy measurement. The final term, $c$, which is significant at lower energies, corresponds mainly to the electronic noise [8].

\subsubsection{Noise-Subtracted Energy Resolution}

The understanding and treatment of the noise ${ }^{10}$ for the present experimental setup result in the noise being subtracted in quadrature from the term on the left-hand side of Equation (5.4). The three-parameter fit can then be reduced to a two-parameter fit by eliminating the noise term such that our fit now becomes:

$$
\frac{\sigma_{\text {Reco }}}{E_{\text {Reco }}}=\frac{a}{\sqrt{E}} \oplus b
$$

The main reason for the subtraction in quadrature of the noise term is that if it were not done, one would be making the assumption that the noise is constant for each run. For the 2003 test beam data this is not the case, as previously mentioned. Performing a threeparameter fit from Equation (5.4) would therefore result in an inaccurate value for the noise term; the same would be true for the stochastic and constant terms. It is important to recall that one is generally interested in the energy resolution however, and not the

\footnotetext{
${ }^{10}$ Refer to Section 4.5
} 
noise-subtracted energy resolution, to which it is referred, since a true measurement will involve both a signal and noise. Nevertheless, it is the noise-subtracted energy resolution that will be predominantly referred to throughout the remainder of the thesis.

The left-hand quantity in Equation (5.5), $\sigma_{\text {Reco }} / E_{\text {Reco }}$ is therefore the noise-subtracted energy resolution. It will sometimes simply be written as $\sigma_{E} / E$ with the same implied meaning. Once measurements have been made for each beam energy such a noisesubtracted energy resolution value is calculated, and a standard $\chi^{2}$ fit can be performed for the two-parameter function in Equation (5.5). In order to compare results throughout the thesis, it will be common to refer to the values of the returned parameters, namely, the stochastic term, $a$, and the constant term, $b$.

The requirements for the noise-subtracted energy resolution for the forward region of the ATLAS calorimeter system for hadrons are given by [4]:

$$
\frac{\sigma_{E}}{E}=\frac{100 \%}{\sqrt{E(G e V)}} \oplus 10 \%
$$

\subsection{Summary of Electron Data Results}

The final summary to be made prior to introducing the specifics of the hadronic weighting schemes is that of the electron data results from the 2003 test beam. These results are summarized in more detail in the aforementioned 2008 publication of test beam data [13], though a brief description is required here. The present analysis is one with a focus on pion data from the $2003 \mathrm{FCal}$ test beam rather than a joint analysis of both electron and pion data. Previous analyses of electron data have been used to calibrate the EM scale 
in units of $\mathrm{GeV}$ per $\mathrm{ADC}$.

It has been explained in Section 2.2 that the total energy deposited by particles or their resultant showers is not read out in a sampling calorimeter. The readout is provided only for the active regions of the calorimeter. That energy which is deposited in the absorbing material is instead compensated for based on the sampling fractions of the calorimeter, and this information is then used to set the final conversion scale between the ADC counts and GeV, the units of energy common to the present analysis. Due to the different design specifications of the three modules of the forward calorimeters, it follows that the sampling fractions will be different.

Table 5.1: Values calculated for each FCal module to convert measured energy values from $\mathrm{ADC}$ to $\mathrm{GeV}$.

\begin{tabular}{|c||c|}
\hline FCal Module & Conversion (ADC/GeV) \\
\hline \hline 1 & 12.0 \\
\hline 2 & 6.1 \\
\hline 3 & 5.4 \\
\hline
\end{tabular}

In principle, one could determine the energy response for each module by performing beam test calibrations with electron beams for each of the modules in isolation. In the case of the forward calorimeters however, only the sampling fraction for the first module was measured ${ }^{11}$. The $\mathrm{ADC} / \mathrm{GeV}$ response for each module was calculated based on simulations of electron data, the electronics, and knowledge of the detector design.

\footnotetext{
${ }^{11}$ That of the second module was also measured, though not to the same degree of precision as the first.
} 
The values are shown in Table 5.1. The calibration constant for the first module was measured to be $12.07 \mathrm{ADC} / \mathrm{GeV}$ based on an analysis of electron data from the 2003 test beam [13]. The values in Table 5.1 were then also used for pion data in order to have measured energy values in units of $\mathrm{GeV}$.

The noise-subtracted energy resolution fit results for electron data from the same 2008 publication, including a measure of the systematic errors, were found to be:

$$
\frac{\sigma_{E}}{E}=\frac{(28.5 \pm 1.0) \%}{\sqrt{E(G e V)}} \oplus(3.5 \pm 0.1) \%
$$

\subsection{Summary of Introductory Sections}

Chapters 1 through 5 have set the necessary framework in order to begin a description of the specific focus of the present thesis: a study of different hadronic calibration schemes based on the pion data collected for the 2003 ATLAS forward calorimeter test beam. A crude examination of distributions such as the one shown in Figure 5.4 for $200 \mathrm{GeV}$ pions clearly demonstrates that the hadronic response requires compensation. In order to obtain a higher and more accurate response, two main calibration schemes in the form of hadronic weights are presented: the modular hadronic weighting scheme in Chapter 6 and the radial hadronic weighting scheme in Chapter 8, with their corresponding results in Chapter 7 and Chapter 9, respectively.

As highlighted in this chapter, the primary benchmarks for the comparison of any results will be both the energy response and the noise-subtracted energy resolution, which will often be referred to in the chapters that follow. 


\section{Chapter 6}

\section{Pion Data Analysis Using Modular Hadronic Weights}

\subsection{Modular Weights as a Simple Weighting Scheme}

The non-compensating nature of the ATLAS forward calorimeters results in a non-linear response to hadronic particles as previously mentioned. It has been shown ${ }^{1}$ that hadronic showers - of which a significant, energy-dependent fraction is also classified as electromagnetic - have several distinguishing features and physical properties, and these can be exploited in order to accurately reconstruct the energy deposited by particles such as pions, whose showers have both a hadronic and electromagnetic component.

If the reconstructed energy for a pion were determined using Equation (5.1), as would be done in the case of an electron, it would amount only to a certain fraction of the

\footnotetext{
${ }^{1}$ Refer to Section 2.5 on the properties of hadronic showers
} 
particle's true energy. In principle then, a simple compensating factor could be applied to the calculated value in order to arrive at the desired beam energy. Such an approach would become complicated, however, by the fact that this compensating factor would itself be a function of the particle's true energy. Clearly one cannot know a priori the compensation factor to apply since it would require the true energy of the particle in the first place. An ideal solution in terms of its ease of implementation would be to apply the same compensation factor to all particles deemed to be hadrons, regardless of their initial reconstructed energy.

In practice of course, in order to determine for which particles the compensation is applied, hadronic particles must first be identified and distinguished from electrons or particles which require no such compensation. Though not a trivial task, this identification can and has been done in ATLAS by making use of shower properties such as the shower depth and width in the calorimeter, given the differences between these quantities as highlighted in Section 2.5. Since hadronic showers penetrate deeper and are broader than purely electromagnetic showers, a lower energy density is expected for the showers resulting from hadronic particles. In this way, the energy density and other so-called shower shape variables can aid in the identification of particles. For the case of the present analysis however, only data from pion runs are studied, and a compensation factor is known to always be required.

The reasoning outlined in the previous paragraph regarding the application of a compensation factor lays the foundation of any hadronic calibration scheme, including those explored in this study. While in principle the crude approach suggested above for compensation in terms of applying a single correction factor would return a value very close 
to the true energy of particles at the chosen energy, it would do poorly for other energy values. Clearly an alternate solution will be required. Moreover, while applying such a compensation or 'weighting' factor, it would be advantageous if the spread in reconstructed energy values - namely the energy resolution, as defined in Section 5.2 - could also simultaneously be reduced.

The simplest weighting scheme examined, and the subject of the present chapter, will be referred to as the modular weighting scheme, whereby a different weight or compensation factor is applied to the reconstructed energy in each of the three FCal modules, with the aim to have the reconstructed energy as close as possible to the true energy, while minimizing the energy resolution.

\subsection{Modular Weight Derivation}

A slight modification to Equation (5.1) will allow the reconstructed energy of a pion, upon application of such modular weights, to be written as:

$$
E_{\mathrm{Reco}}=w_{1} E_{1}^{\mathrm{EM}}+w_{2} E_{2}^{\mathrm{EM}}+w_{3} E_{3}^{\mathrm{EM}}
$$

where $w_{1}, w_{2}$, and $w_{3}$ are dimensionless weights applied to FCal1, FCal2, and FCal3, respectively. The EM superscripts on the energy terms correspond to the fact that the energy in each of the modules is at the EM scale as before. To reflect the fact that this sum is now a weighted value for the reconstructed energy, the superscript has been omitted from the term on the left-hand side.

The purpose of any weighting scheme such as the modular one introduced here is to 
exploit the properties of hadronic showers - in this case one could take advantage of the fact that hadronic showers tend to penetrate deeper into a material as compared with purely electromagnetic showers ${ }^{2}$ by choosing appropriate values for the weights. One can expect a similar response to hadronic particles for the three FCal modules in isolation. The fraction of a particle's energy deposited in the three FCal modules, however, will vary with energy given the logarithmic dependence on initial energy of the longitudinal depth of a hadronic shower. It is this physical property that the modular hadronic weighting scheme can be expected to exploit, since the more hadronic portion of a shower, which necessarily requires a compensation factor, is more likely to be situated in the second and third modules.

Once values for the modular weights $\left\{w_{i}\right\}$ have been determined and applied to the pion data at a particular energy as per Equation (6.1), a distribution of reconstructed energy values will be obtained. A fit can subsequently be performed on the weighted data in order to find the reconstructed energy of the distribution as outlined in Section 5.1.3. The application of the weights is therefore quite trivial, and it only remains to determine how the values of the weights should be chosen.

\footnotetext{
${ }^{2}$ Refer to Section 2.5
} 
The two conditions to be taken into account when selecting the modular weight values are:

1. The reconstructed energy, as determined by the double Gaussian fit performed on the reconstructed energy distribution, should match as closely as possible the true beam energy.

2. The energy resolution - a measure of the spread in the reconstructed energy distribution - should be minimized.

The second of the two requirements above can be expressed as a mathematical function, $S$, to be minimized, which takes the following form for the case of the modular weighting scheme:

$$
S\left(w_{1}, w_{2}, w_{3}\right)=\sum_{k=1}^{N_{\text {events }}}\left(\sum_{\imath=1}^{3} w_{\imath} a_{\imath, k}-E\right)^{2}
$$

The first condition, that of the beam energy constraint, is then satisfied by requiring that:

$$
w_{1}\left\langle a_{1}\right\rangle+w_{2}\left\langle a_{2}\right\rangle+w_{3}\left\langle a_{3}\right\rangle=E
$$

In Equations (6.2) and (6.3), $N_{\text {events }}$ is the total number of events, $a_{\imath k}$ is the amount of energy deposited at the EM scale in the $\imath^{\text {th }}$ module (with weight $w_{\imath}$ ) for event $k, \mathrm{E}$ is the true energy (in this case the beam energy), and $\left\langle a_{\imath}\right\rangle$ is the mean energy from the 
distribution of EM scale energies in the $i^{\text {th }}$ module. For a given run, most values in the expressions above are simply numbers once the sum over the events is carried out, and $S$ is therefore a function only of the set of weights $\left\{w_{\imath}\right\}$; it has therefore been written as such for clarity. The two requirements above have therefore been expressed as a function to be minimized along with one constraint, where the variables of the function are the weights for each module.

We can rearrange Equation (6.3) in terms of $w_{3}$, such that:

$$
w_{3}=\frac{E}{\left\langle a_{3}\right\rangle}-w_{1} \frac{\left\langle a_{1}\right\rangle}{\left\langle a_{3}\right\rangle}-w_{2} \frac{\left\langle a_{2}\right\rangle}{\left\langle a_{3}\right\rangle}
$$

Substituting the expression for $w_{3}$ from Equation (6.4) into Equation (6.2), one finds that:

$$
\begin{aligned}
S\left(w_{1}, w_{2}\right)= & \sum_{k=1}^{N_{\text {events }}}\left(w_{1} a_{1 k}+w_{2} a_{2 k}+E \frac{a_{3 k}}{\left\langle a_{3}\right\rangle}\right. \\
& \left.-w_{1} \frac{a_{3 k}\left\langle a_{1}\right\rangle}{\left\langle a_{3}\right\rangle}-w_{2} \frac{a_{3 k}\left\langle a_{2}\right\rangle}{\left\langle a_{3}\right\rangle}-E\right)^{2}
\end{aligned}
$$

In the present analysis the weight for the third module, $w_{3}$, has been removed using the beam energy constraint. Which of the three weights to remove with the constraint was an arbitrary choice.

One is now in a position to expand Equation (6.5), and sum over all of the included events. This sum includes the events for all runs at a given beam energy. 
Making use of the following conventions:

$$
\begin{aligned}
\left\langle a_{i} a_{j}\right\rangle & =\frac{1}{N_{\text {events }}} \sum_{k=1}^{N_{\text {events }}} a_{i k} a_{j k} \\
\left\langle a_{i}\right\rangle & =\frac{1}{N_{\text {events }}} \sum_{k=1}^{N_{\text {events }}} a_{i k}
\end{aligned}
$$

the function $S$ can be expressed as follows:

$$
\begin{aligned}
& \frac{S\left(w_{1}, w_{2}\right)}{N_{\text {events }}}=\quad w_{1}{ }^{2}\left[\left\langle a_{1} a_{1}\right\rangle-\frac{2\left\langle a_{1}\right\rangle\left\langle a_{1} a_{3}\right\rangle}{\left\langle a_{3}\right\rangle}+\frac{\left\langle a_{1}\right\rangle^{2}\left\langle a_{3} a_{3}\right\rangle}{\left\langle a_{3}\right\rangle^{2}}\right] \\
& +w_{2}^{2}\left[\left\langle a_{2} a_{2}\right\rangle-\frac{2\left\langle a_{2}\right\rangle\left\langle a_{2} a_{3}\right\rangle}{\left\langle a_{3}\right\rangle}+\frac{\left\langle a_{2}\right\rangle^{2}\left\langle a_{3} a_{3}\right\rangle}{\left\langle a_{3}\right\rangle^{2}}\right] \\
& +2 w_{1} w_{2}\left[\left\langle a_{1} a_{2}\right\rangle-\frac{\left\langle a_{1}\right\rangle\left\langle a_{2} a_{3}\right\rangle}{\left\langle a_{3}\right\rangle}-\frac{\left\langle a_{2}\right\rangle\left\langle a_{1} a_{3}\right\rangle}{\left\langle a_{3}\right\rangle}+\frac{\left\langle a_{1}\right\rangle\left\langle a_{2}\right\rangle\left\langle a_{3} a_{3}\right\rangle}{\left\langle a_{3}\right\rangle^{2}}\right] \\
& -2 E w_{1}\left[\left\langle a_{1}\right\rangle-\frac{\left\langle a_{1} a_{3}\right\rangle}{\left\langle a_{3}\right\rangle}-\frac{\left\langle a_{1}\right\rangle\left\langle a_{3}\right\rangle}{\left\langle a_{3}\right\rangle}+\frac{\left\langle a_{1}\right\rangle\left\langle a_{3} a_{3}\right\rangle}{\left\langle a_{3}\right\rangle^{2}}\right] \\
& -2 E w_{2}\left[\left\langle a_{2}\right\rangle-\frac{\left\langle a_{2} a_{3}\right\rangle}{\left\langle a_{3}\right\rangle}-\frac{\left\langle a_{2}\right\rangle\left\langle a_{3}\right\rangle}{\left\langle a_{3}\right\rangle}+\frac{\left\langle a_{2}\right\rangle\left\langle a_{3} a_{3}\right\rangle}{\left\langle a_{3}\right\rangle^{2}}\right] \\
& -E^{2}\left[1-\frac{\left\langle a_{3} a_{3}\right\rangle}{\left\langle a_{3}\right\rangle^{2}}\right]
\end{aligned}
$$

The function $S$ as expressed in Equation (6.8) can be recognized to be in the form of a two-dimensional quadratic function. The various averaging terms expressed in Equation (6.6) and Equation (6.7) can be determined by looping over all of the events for a particular beam energy, such that all of the terms in Equation (6.8) except for the weights 
are known.

The next step is to determine the values of $w_{1}$ and $w_{2}$ in the preceding expression which yield a minimum of the function $S$. Such values could be calculated directly using a calculus-based approach, that is by determining the values of $w_{1}$ and $w_{2}$ which yield a critical point, such that:

$$
\frac{\partial S}{\partial w_{1}}=0
$$

and

$$
\frac{\partial S}{\partial w_{2}}=0
$$

The critical point will be a local minimum provided that both:

$$
\frac{\partial^{2} S}{\partial w_{1}^{2}} \frac{\partial^{2} S}{\partial w_{2}^{2}}-\left(\frac{\partial^{2} S}{\partial w_{1} \partial w_{2}}\right)^{2}>0
$$

and

$$
\frac{\partial^{2} S}{\partial w_{1}^{2}}>0
$$

at the values of $w_{1}$ and $w_{2}$ where the critical point occurs.

It turns out that a far simpler approach is to use a nested loop which sweeps through values of $w_{1}$ and $w_{2}$ in small, fixed increments, ultimately returning the values of the weights which produce a minimum in $S$. Figure 6.1 shows a sample of the function $S$ as a function of the first two modular weights, specifically for the $200 \mathrm{GeV}$ data at point 4L. Although the calculus-based approach will yield a single critical point for this case, 
the presence of a trough region can be seen which demonstrates the negative correlation between the weights. So while there exists a unique minimum value for the function, other values of $w_{1}$ and $w_{2}$ could be selected and applied which would result in a very similar resolution.

The value of the third weight, $w_{3}$, can subsequently be calculated from Equation (6.4). Future analyses, for which a greater number of weights will be involved, will require the use of a more complex minimization algorithm, which should also provide uncertainties on the extracted weight values. It was verified that for the case of modular weights, the simple minimization presented here involving a nested loop did, in fact, return the same values as the more complex minimization algorithm to be introduced in Chapter 8 .

\subsection{Errors on Extracted Weights}

The disadvantage of using the nested loop method mentioned above to determine the weights is that there are no errors returned with the extracted values. An estimation of the errors are determined as outlined below.

The weights are first calculated as summarized above but using only roughly half of the runs. The weight for the $i^{t h}$ module based on these runs is then written as $w_{i, \alpha}$. The procedure is repeated for the remaining runs with the corresponding weight written as $w_{i, \beta}$. Finally, the weights are derived using all of the runs such that the overall value of the weight is:

$$
w_{\imath} \pm \sigma_{w_{\imath}}
$$




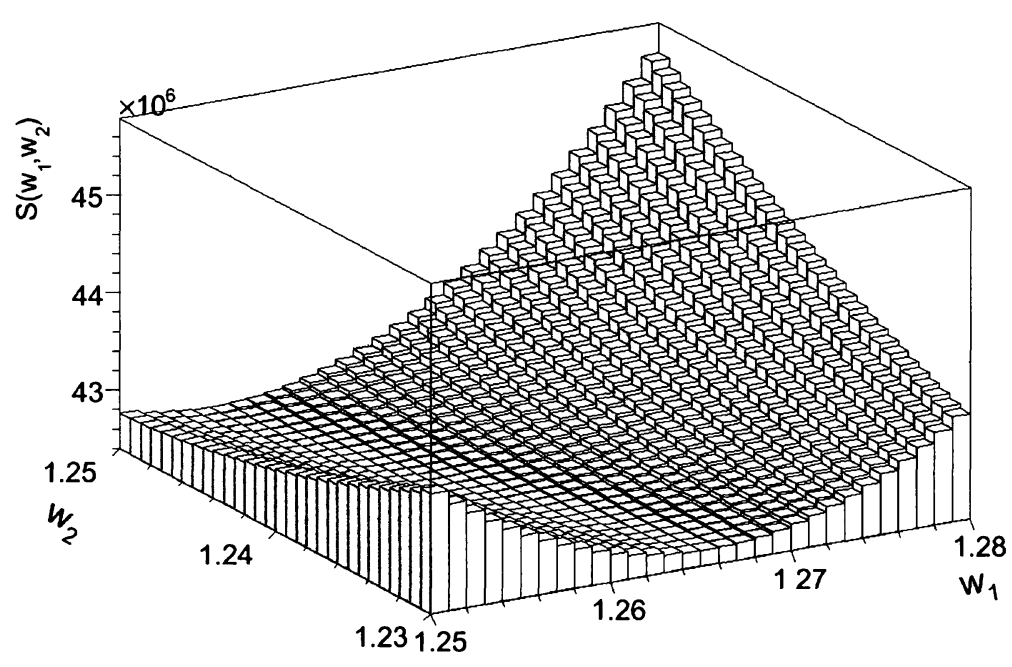

Figure 6.1: A sample plot of $S$ is shown as a function of of the modular weights $w_{1}$ and $w_{2}$. The particular case corresponds to $200 \mathrm{GeV}$ pion data from beam point $4 \mathrm{~L}$. The extracted values for $w_{1}$ and $w_{2}$ were found to be 1.263 and 1.235 , respectively, which can be seen to lie within the trough region as outlined in the text.

where

$$
\sigma_{w_{\imath}}=\left|w_{\imath, \alpha}-w_{\imath, \beta}\right|
$$

The value $\sigma_{w_{\imath}}$ as shown in Equation (6.14) will be used for the errors on the modular weights in the figures which follow. It turns out, in fact, that the more complex weight derivation algorithm which will be introduced in Chapter 8, returns errors on the extracted weights which are quite small. The estimate on the errors as outlined here will therefore also be used to provide a sense of the variation on any extracted weights 
throughout the remainder of the thesis.

\subsection{Modular Weight Values for Point 4L}

Having laid the framework for the weight extraction method, there is also a choice in the cell clustering algorithm which can be used before extracting the weights - either cylindrical or topological clustering ${ }^{3}$.

The extracted weights for each clustering algorithm are presented separately in this section. As fewer cells or readout channels have been included for the topological clustering algorithm, differences are to be expected in the weights based on the two cell clustering algorithms.

\subsubsection{Modular Weights Using Cylindrical Clustering}

Figure 6.2 shows the weight values determined for each of the three FCal modules for beam impact point $4 \mathrm{~L}$, based on the cylindrical clustering of cells. The weights are shown as a function of the true beam energy.

A few comments can be made regarding the trends observed for the extracted values. At lower energies, the expected amount of energy deposited in FCal 2 and FCal 3 should be minimal and correspond almost exclusively to noise fluctuations; the low values of $w_{2}$ and $w_{3}$ will ensure that this noise is suppressed in order to minimize the energy resolution. Also, since nearly all of the energy will be expected to be deposited in FCal 1 at these low energies, the value of $w_{1}$ will have to be considerably higher in order to compensate

\footnotetext{
${ }^{3}$ Refer to Section 5.1.2
} 


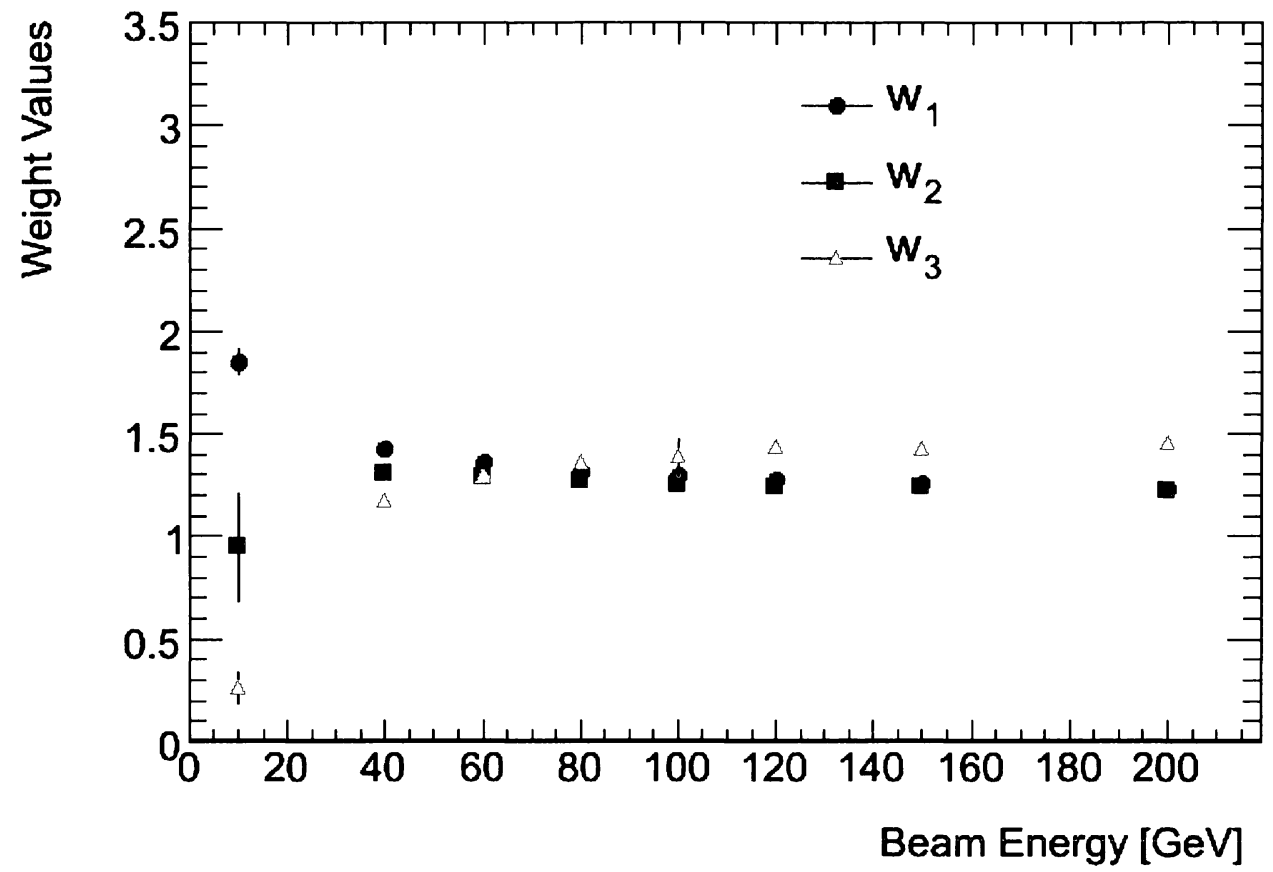

Figure 6.2: Extracted modular weights shown as a function of beam energy for beam impact point $4 \mathrm{~L}$, where the cylindrical clustering of cells was employed. The errors on the weights correspond to the systematic errors presented in the preceding section.

for the fraction of energy invisible to the calorimeter. As the beam energy increases, this fraction of invisible energy in the resulting showers decreases, so the values of $w_{1}$ and $w_{2}$ can be expected to decrease slightly, as is seen to be the case. Finally, at higher energies, the energy in FCal 3 can be expected to be mainly hadronic, and one can note the higher value of $w_{3}$ to account for this fact. 


\subsubsection{Modular Weights Using Topological Clustering}

Weights for each FCal module for point 4L using topological clustering are shown in Figure 6.3, similarly as a function of beam energy. With only three weights per FCal module which may be varied, one can note that the differences are minimal between the results for the cylindrical or topological clustering algorithms.

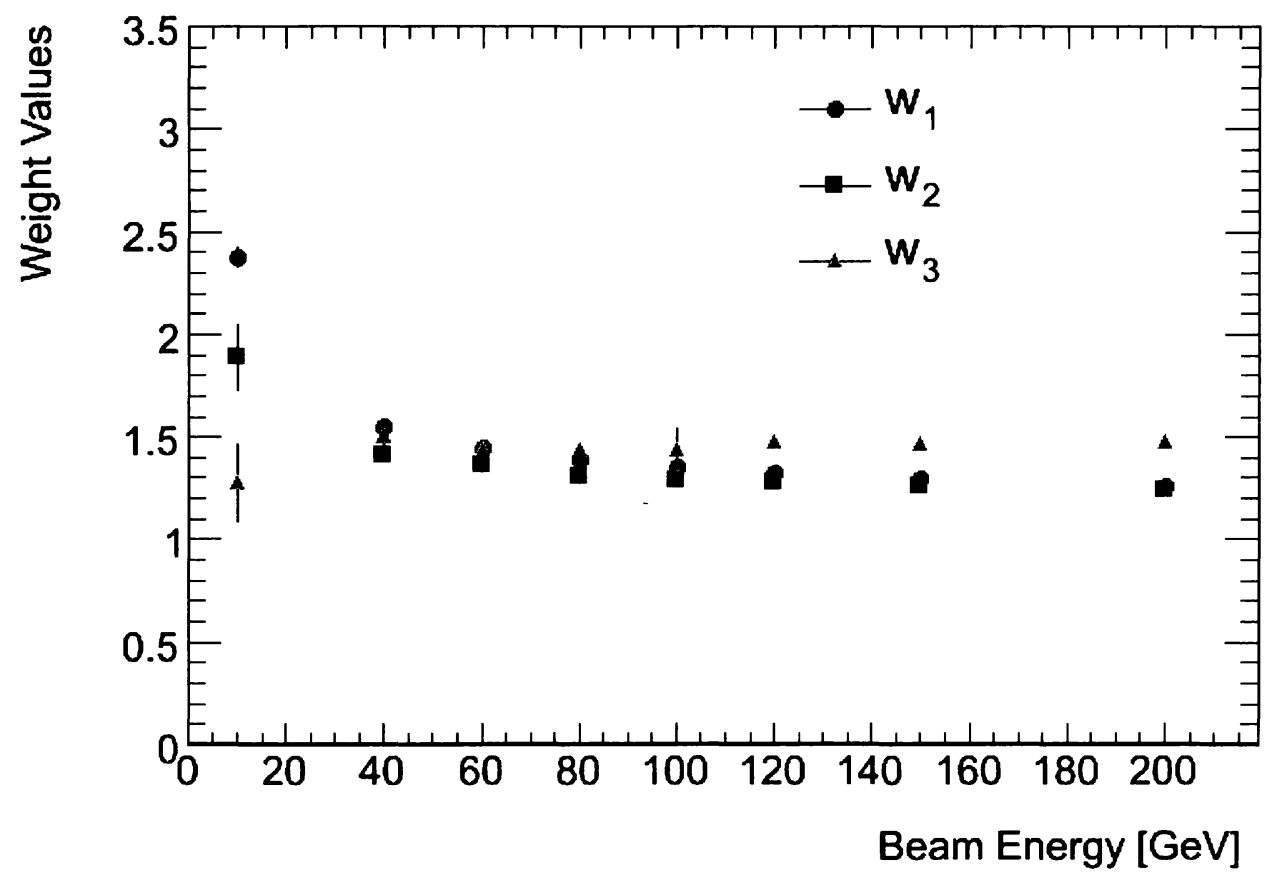

Figure 6.3: Extracted modular weights shown as a function of beam energy for beam impact point $\mathbf{4 L}$, where the topological clustering of cells was employed. Systematic errors are included similarly to the case for cylindrical clustering.

It is notable that the values of the three weights are similar at higher energy, the 
region of most importance to ATLAS analyses.

The most striking. difference between Figures 6.2 and 6.3 is that in the latter case, the value of $w_{3}$ is significantly higher at the lowest beam energy value of $10 \mathrm{GeV}$. Due to the design of the topological clustering algorithm, the noise will be suppressed by simply excluding those cells which correspond primarily to noise and to a broadening of the reconstructed energy distribution; the value of $w_{3}$ will therefore not need to be as low as in the case of cylindrical clustering.

\subsection{Modular Weights For Points 1, 2, 3 and $4 \mathrm{H}$}

Modular weight values were extracted for beam impact point $4 \mathrm{H}$ data and showed only slight differences from the 4L weights at higher energies. Similarly, modular weights were derived for the so-called inner points 1,2 , and 3, though for these points, as will become clear in subsequent chapters, a significant amount of energy is unaccounted for due to the empty region at the centre of the forward calorimeter modules. As a result, the modular weight values in these cases are, unsurprisingly, significantly greater than those extracted for $4 \mathrm{H}$ and $4 \mathrm{~L}$. This fact will be addressed in Chapter 10 in regards to the application of weights to these inner edge points. Modular weights for beam point $4 \mathrm{H}$ data are included in Appendix A.1. 


\section{Chapter 7}

\section{Modular Hadronic Weight Results}

\subsection{Choice of Energy-Independent Weights}

Having derived the modular hadronic weights in the previous chapter, both for the case of cylindrical and topological clustering algorithms, one is now able to apply the extracted weights to the pion data in order to see their effect on the reconstructed energy distributions and the noise-subtracted energy resolution. The result that the weighted reconstructed energy is roughly equal to the beam energy should remain unsurprising given that a beam constraint was used in deriving the weights ${ }^{1}$.

In practice, the application of energy-dependent weights, though it might appear ideal, is of course problematic in that one does not know a prıor the energy of a given particle. Although weights were determined for all beam energy values, in part to develop an intuitive sense for how the extracted weights vary with energy, it was decided to apply

\footnotetext{
${ }^{1}$ Refer to Section 6.4.
} 
the highest energy weights - $200 \mathrm{GeV}$ weights in this case - to all beam energy events.

The choice of $200 \mathrm{GeV}$ weights was of course not arbitrary: when collisions take place at the centre of the ATLAS detector, particles with higher energy, particularly higher transverse energy ${ }^{2}$, will generally be more likely to correspond to an event of interest. Moreover, as has been described in Section 2.7, the need to vary the compensation for the non-EM component of hadronic showers by use of hadronic weights decreases with increasing beam energy. One would therefore expect that applying $200 \mathrm{GeV}$ weights to all beam energy values, the reconstructed energy values for particles with energies higher than $200 \mathrm{GeV}$ should still be quite close to their true energy. For the remainder of this chapter then, it should be assumed, unless otherwise mentioned, that the application of weights to events at any beam energy corresponds to those weights extracted for 200 $\mathrm{GeV}$ events.

It should be mentioned at this point that one would not wish to apply weights to showers which are purely electromagnetic in nature. It is recognized that the present test beam environment is an idealized one in which one has the luxury of knowing the initial energy of each test beam particle, and furthermore that each event, aside from contamination, corresponds to a pion - a hadronic particle. Indeed, the matter of accurately determining for which showers hadronic weights should be applied, and at what point the showers are best left at the EM scale, is currently a topic of great interest in the ATLAS community. This, however, is not the current aim of the present analysis but this simplification should be considered when applying any schemes to standard ATLAS analyses.

\footnotetext{
${ }^{2}$ Refer to Section 1.4.1
} 
Although weights were determined for all beam impact points, point $4 \mathrm{~L}$ has particular interest. With minimal upstream material present, results from this impact point represent the intrinsic response of the FCal. It could be argued that point $4 \mathrm{H}$ would be the more suitable choice as a focus given that the material present upstream of this point more closely resembles the situation which will be present during the collection of ATLAS data. While this is true, the main focus of the present analysis will be on the intrinsic response of the calorimeter as opposed to its response during LHC collisions.

\subsection{Reconstructed Energy}

\subsubsection{Results Using Cylindrical Clustering}

Figure 7.1 shows the reconstructed energy distributions for each of the eight different beam energy values at point $4 \mathrm{~L}$, having applied the $200 \mathrm{GeV}$ weights which were extracted using cylindrically clustered cells. One can note the clear need for the double Gaussian fit $^{3}$, particularly upon examination of the shape of the 150 and $200 \mathrm{GeV}$ reconstructed energy distributions. The results of such a double Gaussian fit from which one obtains the reconstructed energy corresponding to each beam energy are shown in the legends on each plot, along with the RMS value for the distributions which is calculated as the second moment of the double Gaussian function. Also shown in the bottom right of Figure 7.1 is the linearity plot - the reconstructed energy over the beam energy plotted as a function of beam energy. Note that the $200 \mathrm{GeV}$ point is shown to be unity, as one would expect based on the fact that the $200 \mathrm{GeV}$ weights are used.

\footnotetext{
${ }^{3}$ Refer to Section 5.1 .3
} 

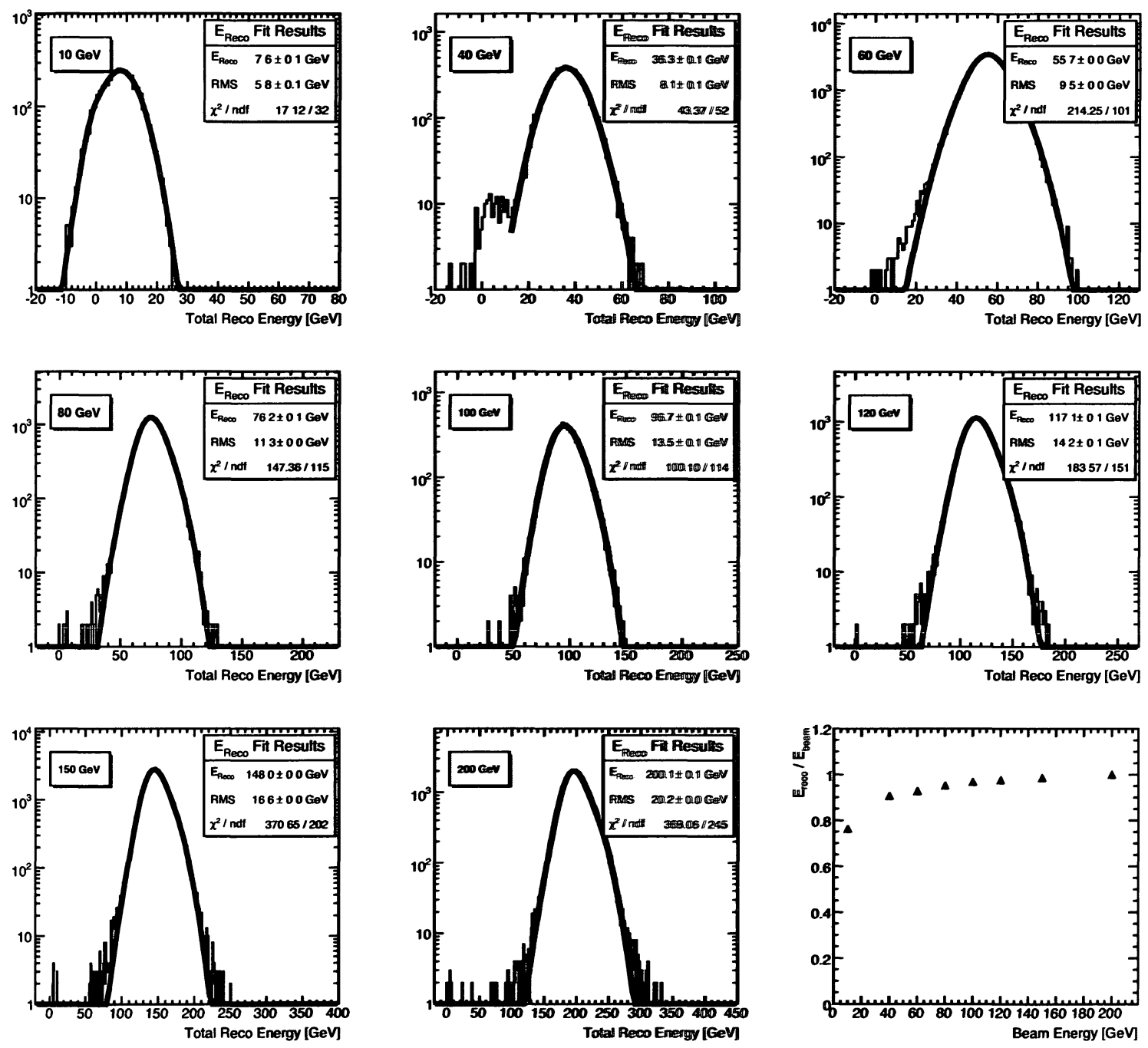

Figure 7.1: Distributions of pion reconstructed energy values for all 4L events which passed the cuts as summarized in Chapter 4. Note that, as is explained in Section 7.1, the $200 \mathrm{GeV}$ modular weights values have been applied at all beam energies. Both data and derived weights are based on the cylindrical clustering algorithm. 

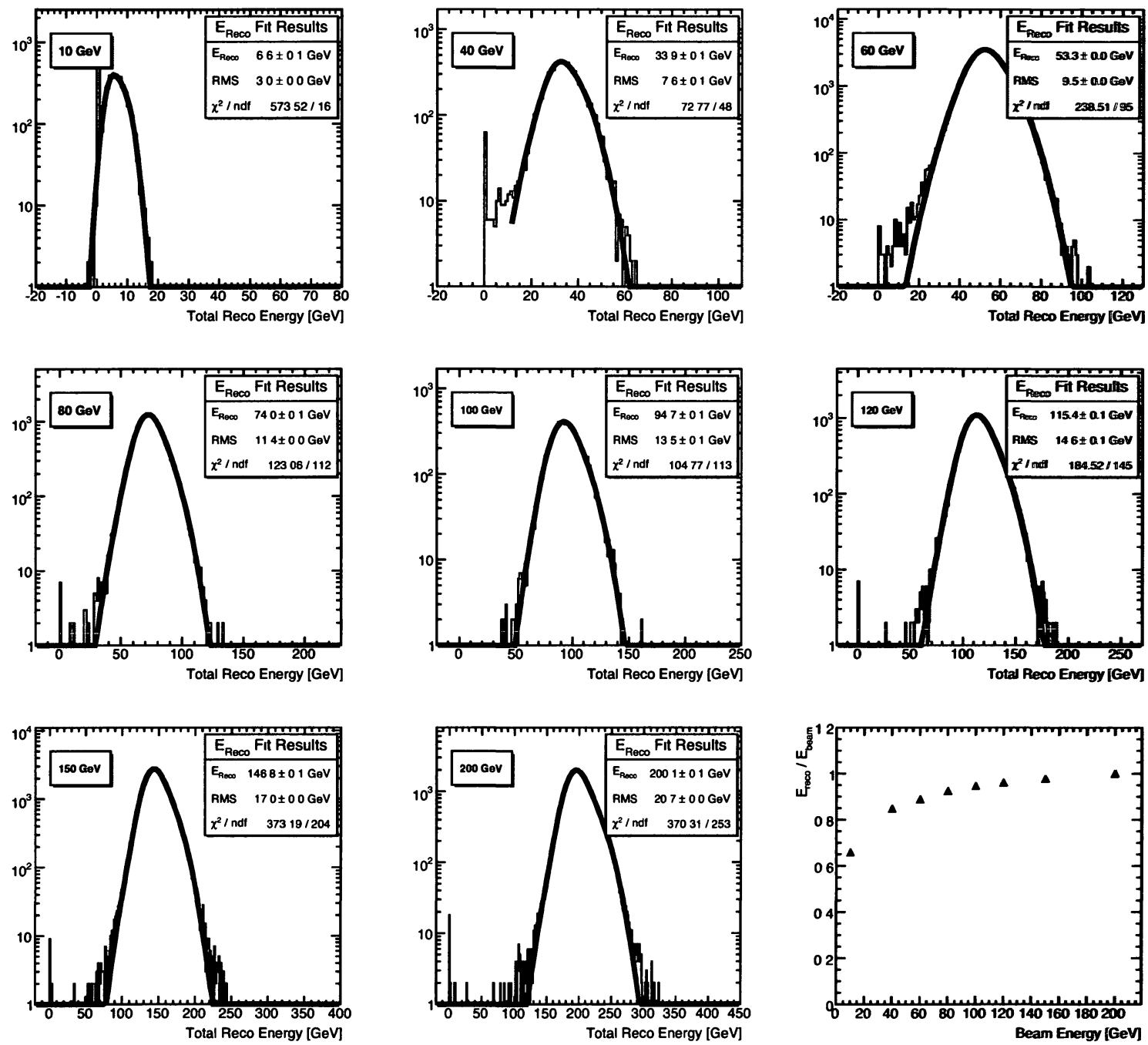

Figure 7.2: Distributions of pion reconstructed energy values for all $4 \mathrm{~L}$ events which passed the cuts as summarized in Chapter 4. In this case, however, the data used and weights derived are as the result of the topological clustering of cells as outlined in Section 5.1.2. 
The reconstructed energy can therefore be seen to be within $10 \%$ of the beam energy for energies as low as $40 \mathrm{GeV}$.

\subsubsection{Results Using Topological Clustering}

Figure 7.2 shows the reconstructed energy distributions for point $4 \mathrm{~L}$ based on the topological clustering algorithm ${ }^{4}$, similar to the distributions from Figure 7.1.

A linearity plot is shown in Figure 7.3 which allows a comparison of the weighted and unweighted reconstructed energies. The results based on both the cylindrical and topological clustering algorithms are shown in the case of weighted data. The EM scale results correspond to the case of cylindrical clustering, since either algorithm could also be used in this case.

The most striking difference between the results for the two clustering algorithms is the fact that there is a smaller width to the reconstructed energy distributions for lower beam energy values using the topocluster algorithm - note in particular the $10 \mathrm{GeV}$ data. This can be reasoned as follows: at lower energies, one expects the relative contribution from the noise to be more significant, and since one of the primary advantages of the topological clustering algorithm is one of noise suppression, a smaller variation is expected in the reconstructed energy in this region. At higher energies, the noise is diminished relative to the signal and this improvement becomes less significant.

One can also note from the linearity plot that when applying the $200 \mathrm{GeV}$ weights to lower energies, the topological clustering results produce a reconstructed energy lower than that for the corresponding beam energy point when using cylindrical clustering.

\footnotetext{
${ }^{4}$ Appendix A.2 shows the results for which no cell clustering was employed
} 


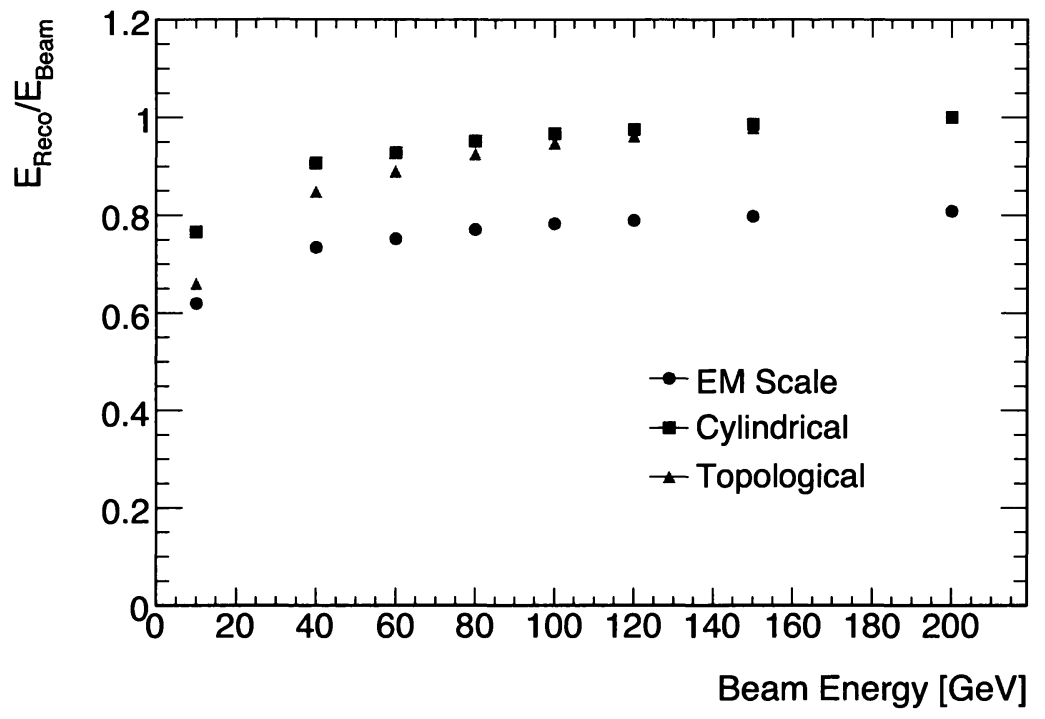

Figure 7.3: Summary of linearity results for pion data at $4 \mathrm{~L}$ with the application of 200 $\mathrm{GeV}$ modular hadronic weights. The results for weighted data both for the cylindrical and topological clustering of cells are shown, as well as the results at the EM scale for the case of cylindrical clustering.

This may be simply explained by the fact that the topological clustering algorithm used includes fewer cells than does the cylindrical clustering algorithm, so one should expect a smaller amount of energy at the EM scale in the case of the former.

\subsection{Noise-Subtracted Energy Resolution}

Having determined the linearity plots based on the reconstructed energy distributions for each clustering algorithm, one can then look at the other benchmark for comparison: the noise-subtracted energy resolution as outlined in Section 5.2.1. 

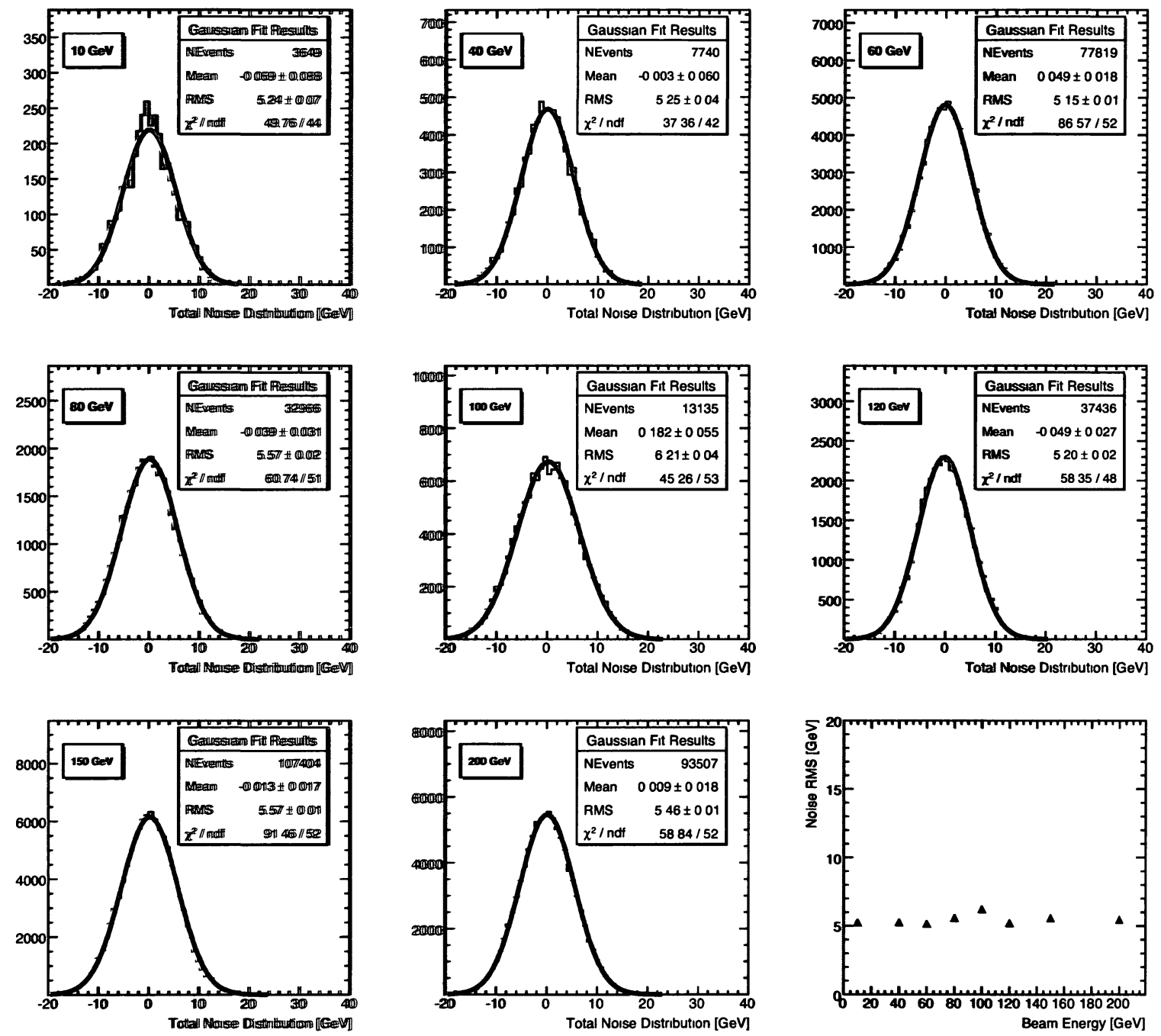

Figure 7.4: Noise distributions with $200 \mathrm{GeV}$ modular weights applied based on the cylindrical clustering of cells. The noise RMS values shown in the lower right as a function of energy are the values which are subtracted in quadrature from the combined signal and noise in order to calculated the noise-subtracted energy resolution. 


\subsubsection{Subtraction of Noise}

One may recall from Section 5.2 that the energy resolution is defined such that the numerator on the left-hand side of Equation (5.5) is the standard deviation of the signal based on the reconstructed energy distribution. In order to obtain this value, one must subtract in quadrature the $\sigma_{\text {noise }}$ from that of the combined standard deviation, which consists of both the signal and noise together. The distributions shown in Figures 7.1 and 7.2 correspond to both noise and signal together. Figure 7.4 shows the noise distributions based on the cylindrical clustering algorithm. Note that for these distributions, as with those for the reconstructed energy, the $200 \mathrm{GeV}$ modular hadronic weights have been applied at all beam energy values.

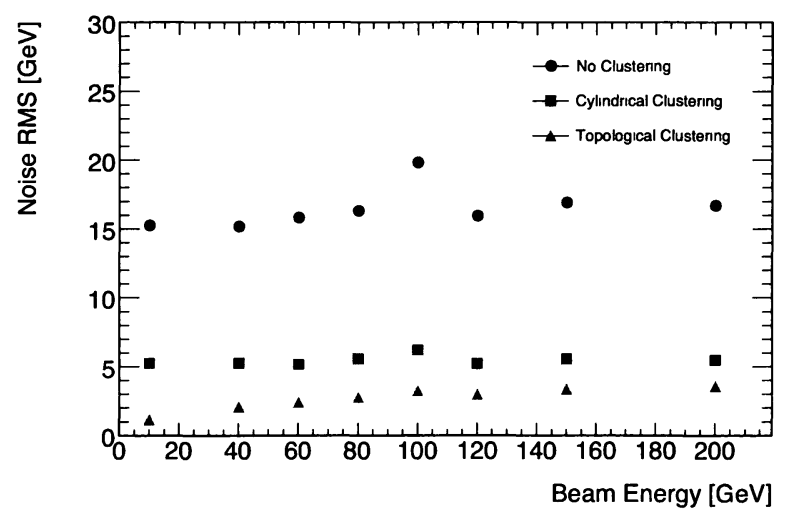

Figure 7.5: Noise RMS values shown as a function of energy based on the inclusion of all cells, as well as for the case of the two noise-suppression cell clustering algorithms.

The same process in the treatment of noise can be used both for cylindrical and topological clustering algorithms. The values of $\sigma_{\text {noise }}$ are compared for the two clustering 
algorithms as a function of beam energy in Figure 7.5. Also shown in this figure are the noise sums when all readout channels active ${ }^{5}$ during the collection of test beam data from all three FCal modules have been included, corresponding to no noise suppression. It is possible to see quite clearly the varying level of noise for each of the three cases. One should remain cognizant of these differences when later comparing noise-subtracted energy resolution values from each of the two algorithms, as one is typically interested in the actual energy resolution as a measure of calorimeter performance, as opposed to the noise-subtracted values, as has been previously mentioned.

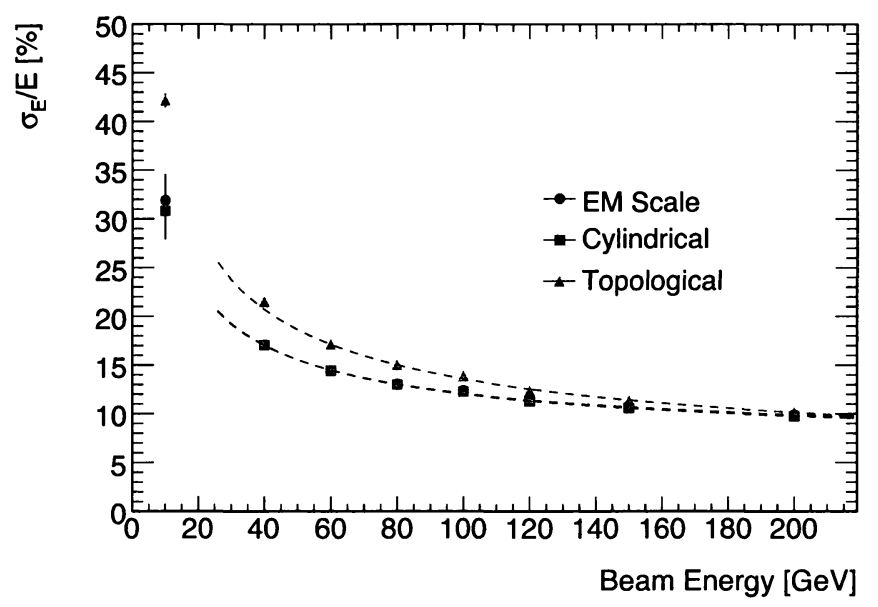

Figure 7.6: The noise-subtracted energy resolution results for both the weighted and unweighted pion data from $4 \mathrm{~L}$ are shown as a function of beam energy. The circular points corresponding to the EM scale results differ only slightly from the cylindrical clustering results for which weights have been applied and as such remain unseen with the exception of the $10 \mathrm{GeV}$ point. The error bars shown correspond to statistical errors only.

\footnotetext{
${ }^{5}$ As shown in Figure 5.1
} 


\subsubsection{Comparison of Noise-Subtracted Energy Resolution}

The calculated noise-subtracted energy resolution values can then be plotted as a function of beam energy, as is done in Figure 7.6 which highlights both the weighted and unweighted (EM) results. Furthermore, one can note the differences between the two cell clustering algorithms.

For both the weighted and unweighted results, the two-parameter fit in Equation (5.5) can then be performed. The fit results are summarized in Table 7.1.

Table 7.1: A summary of stochastic and constant term fit results from 4L pion data, based on the application of derived $200 \mathrm{GeV}$ modular weights. The uncertainty values shown are statistical.

\begin{tabular}{|c|c|c|c|c|}
\hline Weighting Scheme & Clustering & $a\left[\% \cdot \mathrm{GeV}^{\frac{1}{2}}\right]$ & $b[\%]$ & $\sigma_{\mathrm{E}} / \mathrm{E}[\%](200 \mathrm{GeV})$ \\
\hline \hline EM Scale & Cylindrical & $97.8 \pm 0.6$ & $7.10 \pm 0.06$ & $9.90 \pm 0.02$ \\
\hline Modular & Cylindrical & $98.7 \pm 0.6$ & $6.84 \pm 0.06$ & $9.76 \pm 0.02$ \\
\hline Modular & Topological & $127.7 \pm 0.5$ & $4.61 \pm 0.09$ & $10.19 \pm 0.02$ \\
\hline
\end{tabular}

The results based on this fit for the cylindrically clustered data are shown in Equation (7.1) which shows both the stochastic and constant term. The uncertainties from the fit results summarized in Table 7.1 take into account statistical error bars for the data only, and the $10 \mathrm{GeV}$ points are omitted from the fit. The stochastic and constant terms are within the ATLAS requirements highlighted in Equation (5.6).

$$
\frac{\sigma_{E}}{E}=\frac{(98.7 \pm 0.6) \%}{\sqrt{E(G e V)}} \oplus(6.84 \pm 0.06) \%
$$


For comparison, the previous fit results from a 2008 publication, based partly on the analysis of FCal pion test beam data from $4 \mathrm{~L}$, are shown in Equation (7.2). These results, which include systematic ${ }^{6}$ errors, correspond to the same modular weighting scheme as that presented with the use of cylindrical cell clustering [13].

$$
\frac{\sigma_{E}}{E}=\frac{(94.2 \pm 1.6) \%}{\sqrt{E(G e V)}} \oplus(7.5 \pm 0.4) \%
$$

\subsection{Extension to Alternative Weighting Schemes}

In this chapter, one is able to see from the linearity plots that the application of modular hadronic weights to data, based on either clustering scheme, results in a reconstructed energy close to the beam energy over the range of energy values. It is noted that given the minimal improvement in noise-subtracted energy resolution in the case of cylindrically clustered data with modular weights, a common multiplicative factor applied to the energy deposits in all three modules would likely have produced very similar results.

In light of this, it should be pointed out that the scheme outlined in this chapter is the first attempt at a hadronic weighting scheme, and it provides a benchmark with which one can later compare more complex weighting schemes. It was also the primary weighting scheme employed for the results of the 2008 publication based on analyses of both electron and pion data in the 2003 FCal test beam [13], which make this a necessary first step for the purposes of validation and motivation. In principle however, the general methods outlined in both this and the preceding chapter can be applicable

\footnotetext{
${ }^{6}$ The systematics take into account the variation due to the application of different sets of extracted weights as well as the variations based on the fitting algorithm.
} 
to any hadronic weighting scheme.

The radial hadronic weighting scheme to be presented in the following chapter will therefore use the same principles outlined in Chapter 6, and one could in principle use a large number of hadronic weights, provided that the particular weights can be extracted and applied in a reasonable time. The question arises as to whether the addition of a greater number of weights will markedly improve the results. In the interest of computing time and ease of applicability to global ATLAS analyses, one would prefer to adopt a weighting scheme that is as straightforward and simple as possible. 


\section{Chapter 8}

\section{Pion Data Analysis Using Radial Hadronic Weights}

\subsection{Radial Weighting Scheme As a Next Approach}

With the basic structure of the modular weighting scheme in mind, the next step in complexity for a weighting scheme can be explored. In what will be referred to as the radial weighting scheme, motivated by previous analyses [21], the energy deposits in the various FCal readout channels are assigned to radial bins based on the particular readout channel's radial ${ }^{1}$ distance from the impact position, as illustrated by Figure 8.1. Just as different weights are applied to each of the FCal modules in the modular weighting scheme, the present scheme assigns a different hadronic weight to the data in each of the radial bins. The choice of such a setup is to attempt to take advantage of both the

\footnotetext{
${ }^{1} i . e$. in the $x-y$ plane as defined for the ATLAS coordinate system.
} 


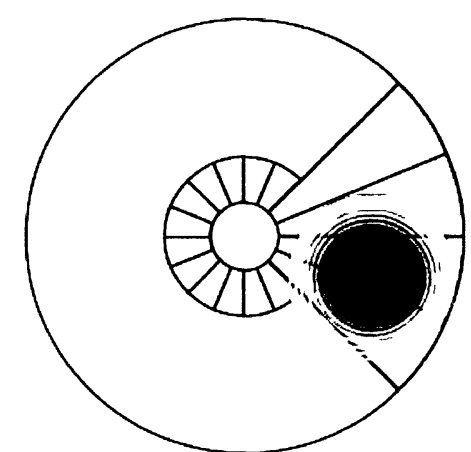

FCal1C

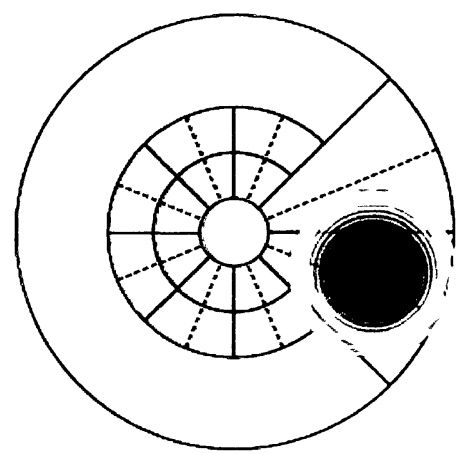

FCal2C

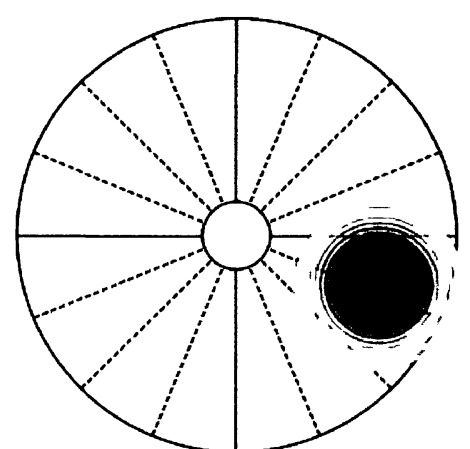

FCal3C

Figure 8.1: A visual representation showing the radial binning of the cells, of which only those shown in grey were included for the 2003 Test Beam. As has been depicted above, one may note that the centre of the concentric radial annular regions may shift, as determined by the tracking information, from module to module.

radial properties of hadronic showers, as well as the fine lateral segmentation of the FCal readout channels.

To determine the bin to which a particular readout channel or cell is assigned, the projected $(x, y)$ position of the beam particle on the surface of each FCal module for a given event is first determined and set to be the centre of the concentric radial bins. This projected position on the front surface is determined based on the BPC tracking information $^{2}$ and it should be emphasized that, as was the case with the cylindrical clustering algorithm, the angular incidence of the particles with respect to the module face will result in a different projected impact position for each of the three FCal modules.

\footnotetext{
${ }^{2}$ The BPC tracking information is initially used, though it is not strictly required as will be made clear in Section 8.3.3
} 
For a given readout channel, its radial distance for the purposes of binning is defined to be the distance between the cell's centroid and the impact position for that module. Equal widths of $1 \mathrm{~cm}$ were chosen for the radial bins which form concentric annular regions around the central point.

In terms of binning offsets, the first bin includes all entries within a radial distance of $1 \mathrm{~cm}$ from the projected impact point. Though only shown when applicable, there is in fact a $17^{\text {th }}$ radial bin, corresponding to the overflow bin. The energy deposit is identically zero for this overflow bin for the case of cylindrical clustering since the clustering algorithm only includes cells up to $16 \mathrm{~cm}$ away from the projected impact point. For the topocluster clustering algorithm however, this is not the case since there may be non-zero contributions at any distance from the impact point, though one would expect a smaller amount of energy far from the impact point. This point will arise at a later point when looking at the number of included topological clusters for a given event.

Indeed, the radial binning presented thus far is quite similar to the setup of the cylindrical clustering algorithm presented in Section 5.1.2 and the radially binned data clustered in this way can therefore continue to be referred to as such. A counterpart to the setup based on the topocluster algorithm can also be defined in a similar way - cells are only included provided they pass the 4/2/0 thresholds as laid out in Section 5.1.2, at which point they are again subdivided into the radial bins as previously described. The same two leading clustering algorithms as those in the case of the modular weighting scheme are therefore employed again once the radial binning has been incorporated into the analysis.

The radial binning of data allows for an investigation of the transverse development 
of energy deposit which was not previously available. In addition to grouping the energy deposits into radial bins, variables were also created containing the number of cells in each radial bin, as well as the noise. Initially no weights were applied to each radial bin so the energy and noise variables were set to be at the EM scale.

It is necessary to assign the noise to radial bins in addition to the energy deposit as the noise will have the same radial weight applied to it, and will subsequently be used in order to determine the noise-subtracted energy resolution as was done in the previous chapter. The number of cells included in a given radial bin however, though not strictly required for the analysis, is helpful, together with the energy and the noise, in developing a feel for the lateral development of hadronic showers.

\subsection{A Look at Radial Profiles}

\subsubsection{Number of Included Readout Channels}

The first quantity of interest with the radial variables in place is the number of included cells and how this number changes as a function of radial distance from the impact point. Such radial profiles for $4 \mathrm{H}$ and $4 \mathrm{~L}$ were found to be quite similar. As such, only those for $4 \mathrm{~L}$ are shown in Figure 8.2. All plots shown in this section include the same standard cuts as introduced previously and show only average values over all events passing the cuts for all data runs at a given beam energy. Statistical error bars have been included for these average values in the plots though are negligible.

Figure 8.2(a) shows the average number of cells included in each radial bin, summed over all FCal modules, based on the cylindrical clustering algorithm. Data points are 
shown for 10,100 and $200 \mathrm{GeV}$, though, as one would expect, the number of cells included for cylindrical clustering of cells does not depend on energy. One can note the difference, however, when the topocluster algorithm is employed, as shown in Figure 8.2(b). As expected due to the nature of the clustering algorithm with its energy to noise thresholds, fewer cells overall are included at lower energies.

It is important when examining plots such as those in Figure 8.2 to note that the effective cross-sectional area of each radial slice is not constant. The true geometry, however, is such that provided the border regions ${ }^{3}$ are ignored and if one assumes all of the readout channels to be equal in size, one expects a roughly linear increase in the cross-sectional area with increasing radial bin number ${ }^{4}$.

In reality of course, the two assumptions regarding the border regions and equal cell sizes are not always valid and must at times be taken into account. If one could ignore them however, one would expect a linear relationship between the number of cells included and the radial bin number, which is roughly what is seen in Figure 8.2(a). One will note that the slope in this plot appears to increase at a radial distance of roughly 12-14 cm. Based on the position of point $4 \mathrm{~L}$, this distance corresponds to the region approaching the inner radius of the FCal, where the cell sizes are known to decrease. It makes sense, therefore, that more cells would be included in this region.

\footnotetext{
${ }^{3}$ For example the inner beam pipe or outside the FCal region, for which there are no active readout channels

${ }^{4}$ This is shown in Appendix B
} 


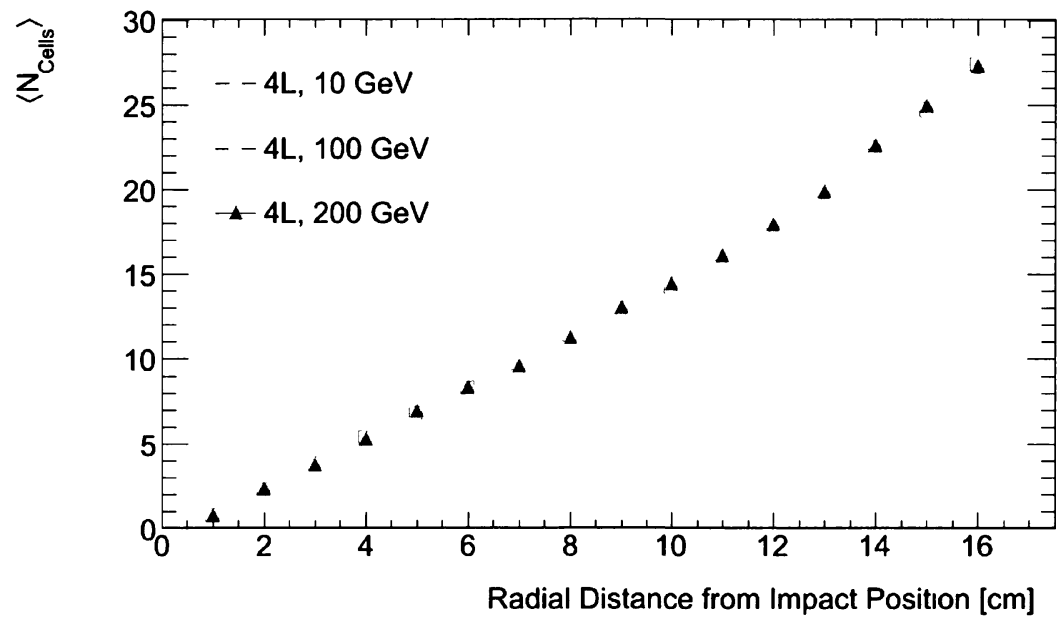

(a) Average number of cells for pion data at $4 \mathrm{~L}$ based on cylindrical clustering

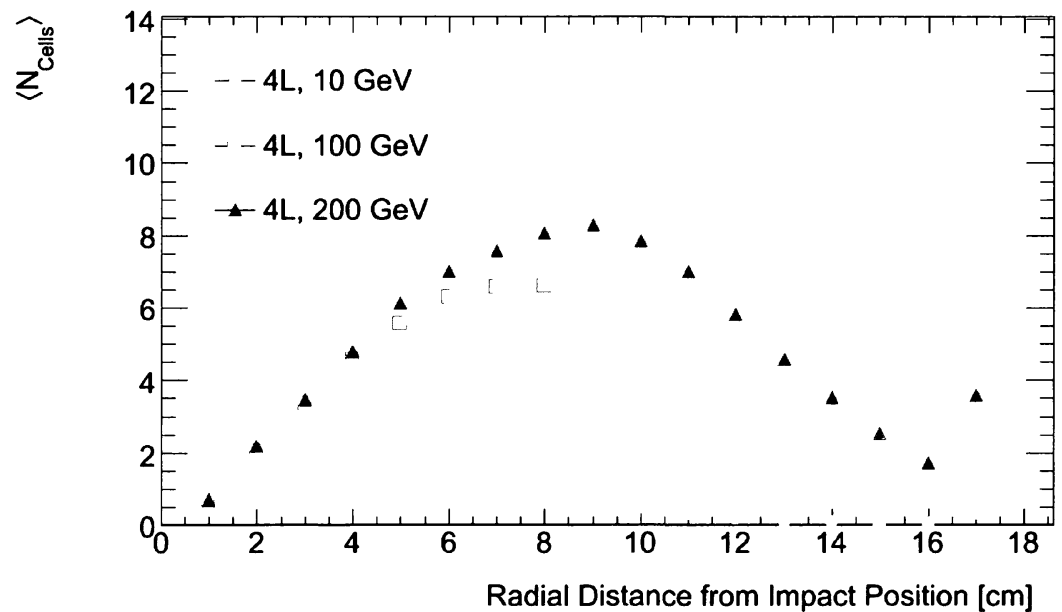

(b) Average number of cells for pion data at $4 \mathrm{~L}$ based on topological clustering

Figure 8.2: Radial profile showing number of included cells with beam impact point $4 \mathrm{~L}$ for a) cylindrical clustering and b) topological clustering. Shown on each plot are the average number of cells included for beam energies of 10,100 , and $200 \mathrm{GeV}$. All three FCal modules are included. 


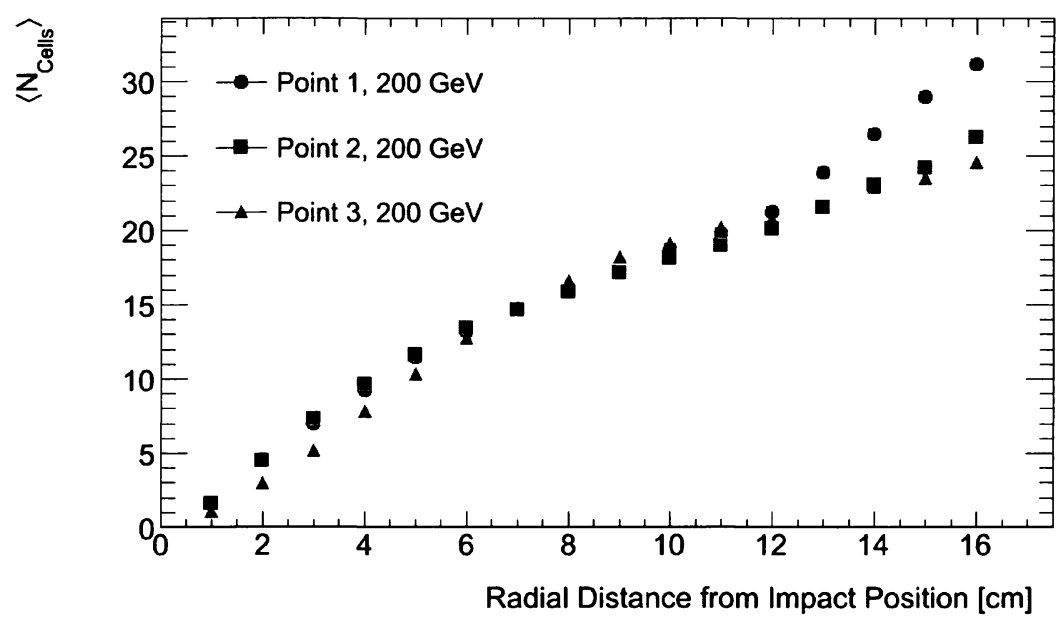

(a) Average number of cells for $200 \mathrm{GeV}$ pion data at inner points based on cylindrical clustering

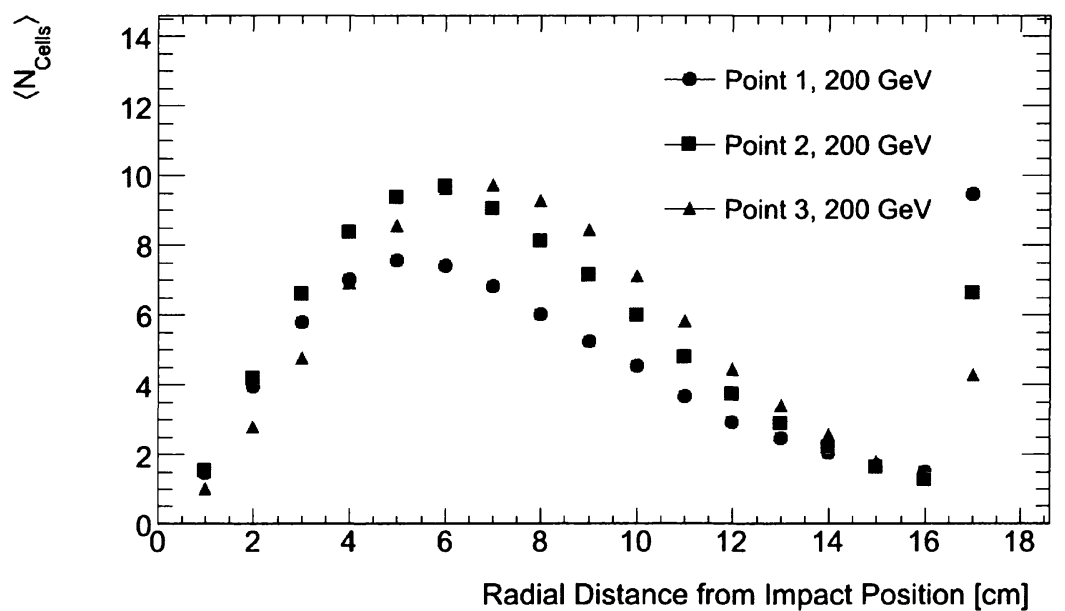

(b) Average number of cells for $200 \mathrm{GeV}$ pion data at inner points based on topological clustering

Figure 8.3: Radial profile showing number of included cells with the three inner beam impact points for $\mathbf{a})$ cylindrical clustering and b) topological clustering. Note that all points correspond to $200 \mathrm{GeV}$ pion data. 
Figure 8.3 shows similar radial profile plots for the inner beam impact points 1, 2, and 3. For the case of cylindrical clustering in Figure 8.3(a), one is again able to see how the trends in the data points correspond to the particular geometry of the FCal setup. One of the more prominent features of this plot is the fact that the slope of the linear trend for each point decreases as opposed to increases in the case of the outer points. This can be reasoned by the fact that once a certain radial distance is reached from each of the inner points, far fewer cells will be included due to the effective area encompassing the central region of the FCal modules, for which no readout channels are present.

The drastic increase in the number of cells for beam point 1 at larger radial distances corresponds to the collection of cells at the other side of the beam pipe. The same can be observed for beam point 2 in the plot, though this happens at a radial distance approximately 4-5 cm further away - roughly the same distance as that between beam points 1 and 2 in the actual FCal geometry. The presence of a significant number of included cells in the overflow bin, as shown at $17 \mathrm{~cm}$ in Figure 8.3(b), can be justified by the likely presence of at least one additional cluster on the opposite side of the beam pipe, as determined by the topological clustering algorithm.

\subsubsection{Energy Density}

Radial profile plots were originally generated showing the amount of energy in $\mathrm{GeV}$ deposited in each radial bin, though this provided a misleading representation of the lateral shower shape, given that the area of each bin is increasing roughly linearly with radial distance, as alluded to earlier. It would then become an onerous task to determine which of the two processes - the changing annular area, or the increase or decrease in 
the amount of deposited energy - to be the reason for the particular trend in the radial profile.

For this reason it was decided that the amount of energy deposited in $\mathrm{GeV}$ would then be divided by the effective volume of those cells included based on the particular clustering scheme - the denominator having been calculated by cumulatively adding the volumes of each of the included cells. For the case of cylindrical clustering, simply using the geometrical volume for each radial bin - its annular area multiplied by the length of the FCal module - would likely result in a physically realistic energy density value. For topological clustering however, one can imagine the extreme case of only one cell being included in perhaps one of the final radial bins; to include the entire annular area of that bin would therefore result in an inaccurate representation of the energy density. The volume in question, therefore, is based on the volume of all of the included cells within a given radial bin, for which the information is provided in the database.

Figure 8.4 shows the average energy density, as defined in the preceding paragraph based on the effective volume, as a function of radial distance from the projected impact position. Figure 8.4(a) takes into account the inclusion of the energy deposits and the effective volumes for the cells in all three FCal modules and is shown at the EM scale with the topological clustering of cells. The remaining figures allow for a comparison of the same energy density radial profiles for each of the FCal modules separately. 


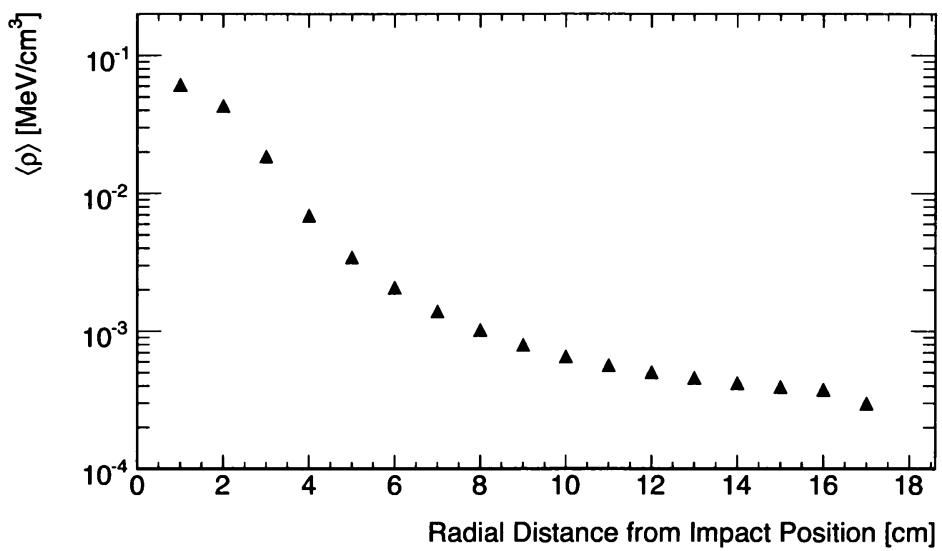

(a) Average over all FCal modules

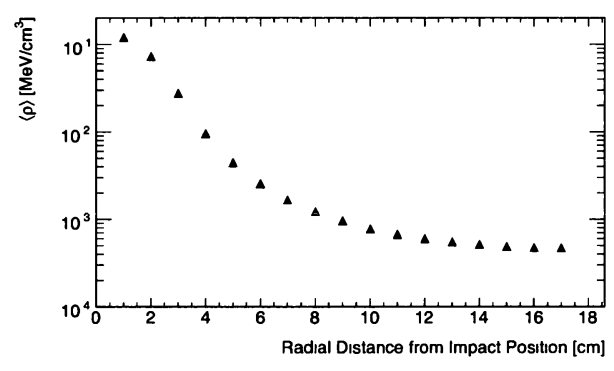

(b) FCal 1

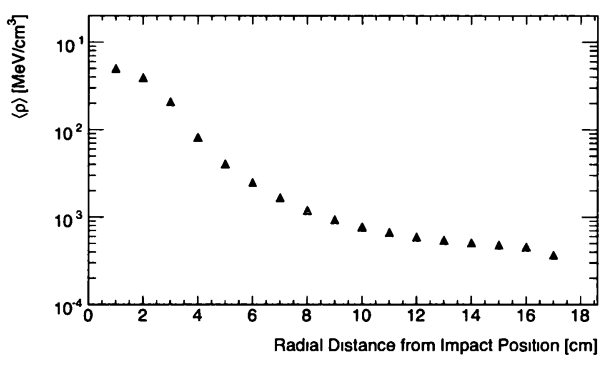

(c) FCal 2

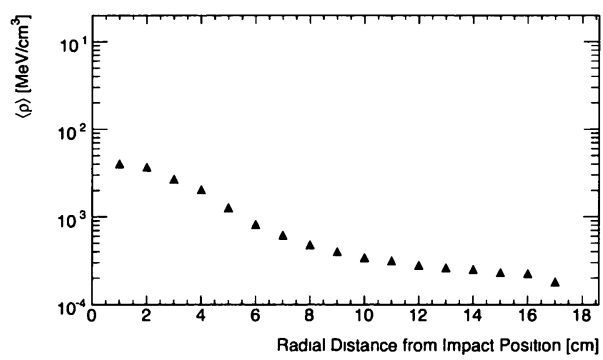

(d) FCal 3

Figure 8.4: EM scale energy density radial profiles for $4 \mathrm{~L} 200 \mathrm{GeV}$ pion data. The topological clustering of cells is used. The individual plots show a) the average over all FCal modules, as well as the the individual radial profiles in b) FCal 1, c) FCal 2, and d) FCal 3. 


\subsection{Deriving Radial Weights}

Having developed a feel for the lateral shower shapes based on the pion data, the next step is to determine, by similar reasoning as that used for the modular weight derivation in Chapter 6, values for the radial hadronic weights which can subsequently be applied to the data to see if there is an improvement in either the linearity or the energy resolution.

Three variations of the radial weighting scheme will be presented separately, and the extracted weights from each will be subsequently applied to the pion data in the following chapter, along with the corresponding results.

\subsubsection{Radial Weights: Standard Setup}

In what will be referred to as the standard setup, the energy deposited in a given module is binned into 16 annular regions as described in the general introduction of this chapter. Equation (8.1) expresses the form for the reconstructed energy for a particular event based on this setup, where $E_{\imath, \jmath}^{\mathrm{EM}}$ and $w_{\imath, \jmath}$ are the EM scale energy and the corresponding radial weight, respectively, for the $i^{\text {th }} \mathrm{FCal}$ module and the $j^{\text {th }}$ radial bin.

$$
E_{\mathrm{Reco}}=\sum_{\imath=1}^{3} \sum_{\jmath=1}^{16} w_{\imath, \jmath} E_{\imath, \jmath}^{\mathrm{EM}}
$$

The $16^{\text {th }}$ bin includes all contributions beyond $15 \mathrm{~cm}$ from the projected impact point on the surface of the corresponding module; that is to say that there were a total of 48 radial weights in the standard setup and not the 51 had a $17^{\text {th }}$, overflow bin been assigned its own weight. 
The radial weights were then determined for a given beam energy value and beam point by first setting up a function $S$, analogous to that expressed in Equation (6.2). The particular form for the function $S$ in the present case is:

$$
S=\sum_{k=1}^{N_{\text {events }}}\left(\sum_{\imath=1}^{3} \sum_{\jmath=1}^{16} w_{\imath, \jmath} a_{\imath, \jmath, k}-E\right)^{2}
$$

where it is recognized that $S$ is a function of all of the radial weights. Analogous to the term $a_{\imath, k}$ in Equation (6.2), $a_{\imath, \jmath, k}$ in the above expression corresponds to the total EM scale energy deposit for event $k$, in module $i$ and radial bin $j$.

The main difference with the new function, of course, is that it would be a function of 48 variables, or rather 47 once the beam constraint is applied, as opposed to the three (two) variables used in the case of the modular weight setup. It behaves, however, in a very similar fashion and is also quadratic in form. In fact, it is unsurprisingly quite similar in form to a standard $\chi^{2}$ function. Suffice it to say, the crude minimization algorithm making use of nested loops such as that used for the modular weight extraction would not be computationally feasible in the present case.

The minimization of such a function of 47 variables was performed using a program written in $\mathrm{C}++$ which returns a parabolic error estimation on each of the radial weight values in addition to the weights themselves [22]. At the beginning of the execution of the program all radial weights are initially set to have a value of 1.0 corresponding to the EM scale but they are subsequently all multiplied by a common factor such that the beam constraint is satisfied. At this point an iterative, stepwise process begins whereby the value of each of the weights is adjusted by a small amount with the value of the $S$ 
function to be minimized recalculated at each step. Subsequent steps are taken until such time as a convergence is reached. The radial weight values yielding a minimum value are then stored in an output file. The program used allowed for a general number, $n$, of weights to be used, though minor alterations to the algorithm and its code were made for the case of the particular setup of interest. It should be mentioned that negative weights can be returned at times, though the structure of the program is such that they are avoided when possible.

The values of the $200 \mathrm{GeV}$ radial weights for data from beam point $4 \mathrm{~L}$ are shown in Figure 8.5. Cylindrical clustering of cells is employed for the weights shown, and the errors as described in Section 6.3 have also been included. It can be seen that the weights for FCal 3 have values slightly below unity in the final radial bins. With relatively little significant energy deposited in this region even at $200 \mathrm{GeV}$, the energy in these bins will correspond predominantly to noise. The suppression of the contributions from these bins by means of a lower weight will thus improve the energy resolution.

Figure 8.6 shows the same extracted weights based on the standard setup but for the case of topological clustering. Larger fluctuations in the extracted radial weights for FCal 1 can be observed in the case of topological clustering particularly at larger radial distances.

The extracted radial weights for the remaining beam points are included in Appendix A.3 for comparison.

Returning to beam point $4 \mathrm{~L}$ however, based on the rough trends of the extracted radial weights shown for $200 \mathrm{GeV}$, as well as those observed at other beam energy values, it was decided to set up an intermediate level of complexity between that for the modular 


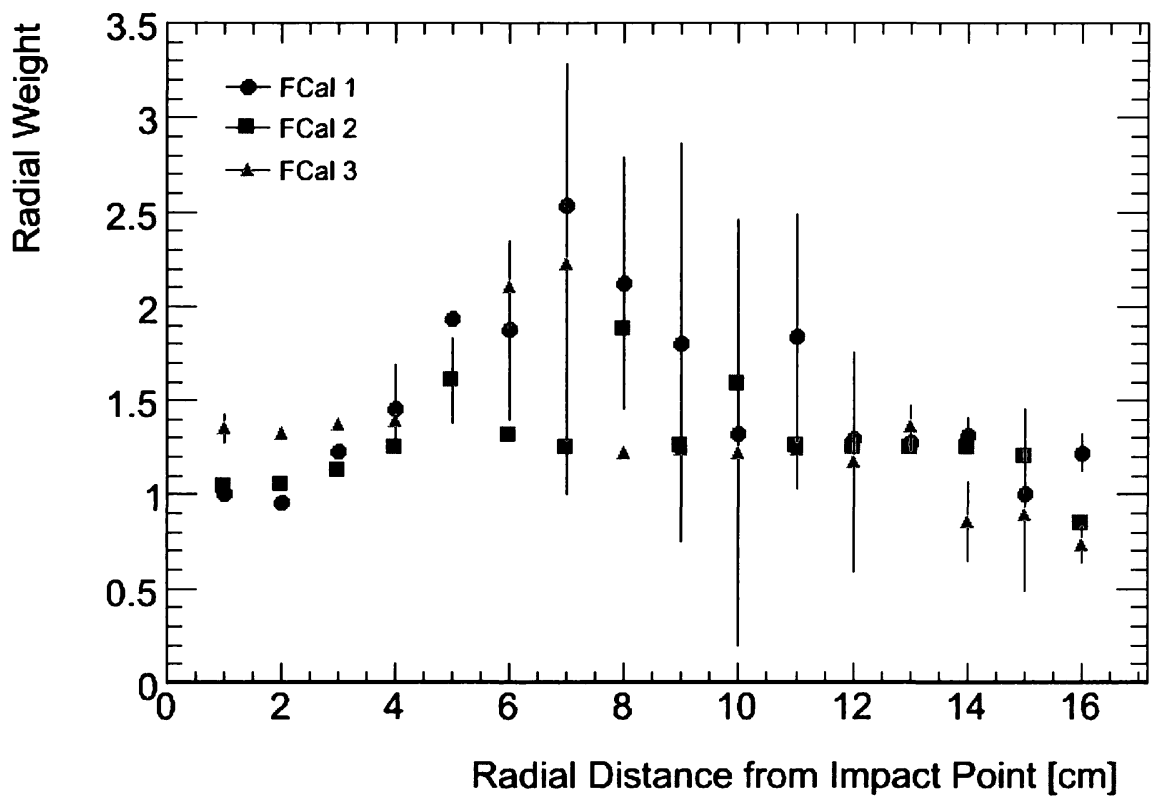

Figure 8.5: Extracted radial weights from $200 \mathrm{GeV} \pi$ data at beam impact point $4 \mathrm{~L}$ based on the standard setup with 16 weights per module. The weights are shown for each FCal module individually as a function of radial distance from the impact point for that module, based on the cylindrical clustering of cells.

weighting scheme and the more complicated standard setup of the radial weighting scheme employing 16 hadronic weights per module. The reason for the inclusion of the second radial weighting scheme was to see if similar results could be obtained with an algorithm employing fewer weights - one which would therefore prove simpler to implement in ATLAS analyses. Though the results have yet to be summarized in the following sections, all variants of the radial weighting scheme will be first be summarized, along with their corresponding extracted weights, and the results will then be compared together in the 


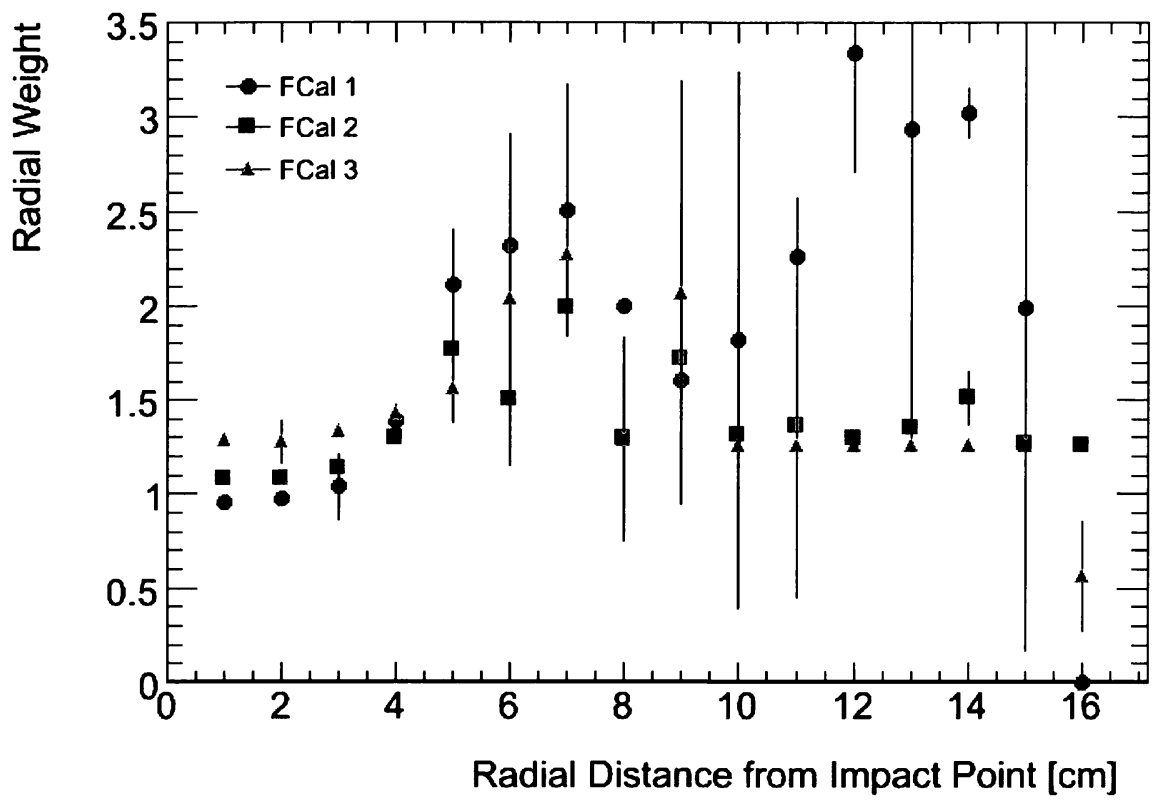

Figure 8.6: Extracted radial weights from $200 \mathrm{GeV} \pi$ data at beam impact point $4 \mathrm{~L}$ based on the standard setup with 16 weights per module. The weights are shown for each FCal module individually as a function of radial distance from the impact point for that module, based on the topological clustering of cells.

following chapter.

It should be noted before moving on that if the modular weight values from the preceding chapters are applied to the data in all radial bins of the appropriate module, the same results as those from Chapter 7 are recovered. With the addition of more degrees of freedom by allowing for 16 radial weights per module, one would hope to obtain results which are improved from or at least equal to the modular results in Chapter 7 in terms of linearity and resolution plots. 


\subsubsection{Radial Weights: Simplified Setup}

The simplified radial weighting scheme reduces the number of radial weights per module to three as opposed to 16 in the standard setup. It was decided to define the sizes of the three bins based on the following reasoning, again motivated by the observed trends in the extracted radial weights from the standard setup:

- The innermost region encompasses the first three of the standard setup bins. One would expect the weights for this region to have slightly lower values due to the fact that it should contain a larger EM shower component.

- The second or middle region includes the next nine bins from the standard setup and is expected to have slightly higher values to increase the weight of the purely hadronic component of the resulting showers.

- Finally, the outermost region in the simplified setup contains the four outermost bins from the standard setup in addition to the overflow bin. For the case of cylindrical clustering, one would expect this outermost bin to have slightly lower values, particularly in the third module, in order to suppress what one would expect to be predominantly noise.

A visual representation highlighting the differences between the binning in the standard and simplified setup is shown in Figure 8.7. The extracted $200 \mathrm{GeV}$ weights based on the simplified setup from 4L data are shown in Figure 8.8 and Figure 8.9 for the cases of cylindrical and topological clustering, respectively. It can be seen that the third and final weights for all three modules are higher in the case of topological clustering, which 


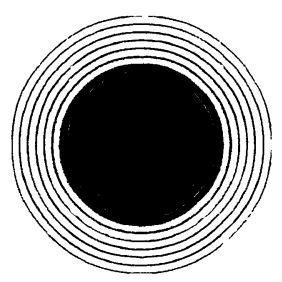

(a) Standard setup

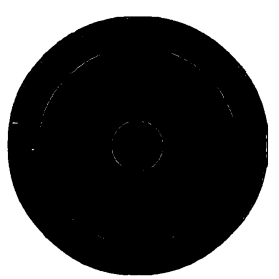

(b) Simplified setup

Figure 8.7: Visual representation of the different radial binning used in the radial weighting setup. Shown are both a) the standard 16 bins per FCal module, and b) the simplified setup with only three weights per FCal module as outlined in the text.

one would expect given that fewer cells have been included in this region, so those cells which are included in clusters will have to be weighted more heavily. Also, the weights for the first bin in the first two modules can be seen to be close to one but for the second bin they are higher since in the latter case one expects the showers to be more hadronic-like.

As in the case of the standard setup, extracted weights for the simplified setup for the remaining beam points are included in Appendix A.3. 


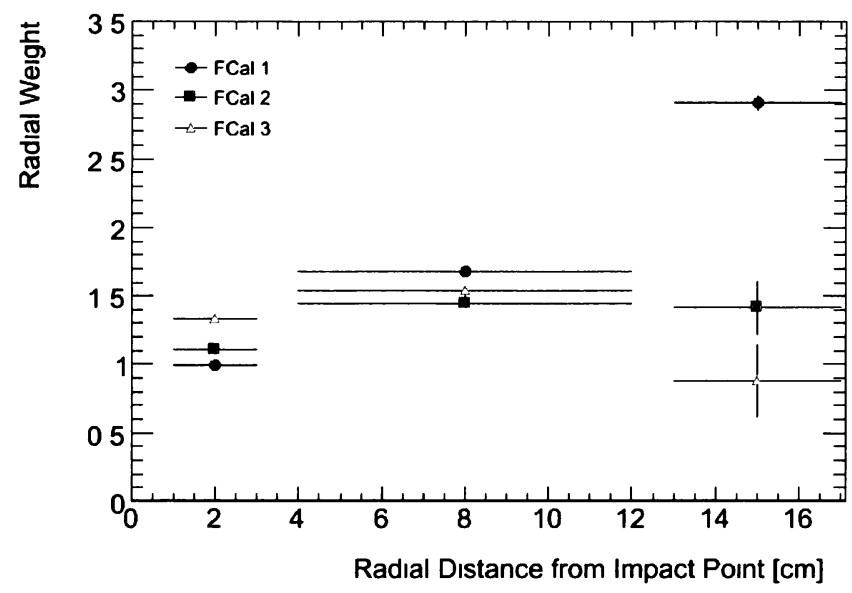

Figure 8.8: Extracted radial weights from $200 \mathrm{GeV} \pi$ data at point $4 \mathrm{~L}$ based on the simplified setup with 3 weights per module and based on the cylindrical clustering of cells.

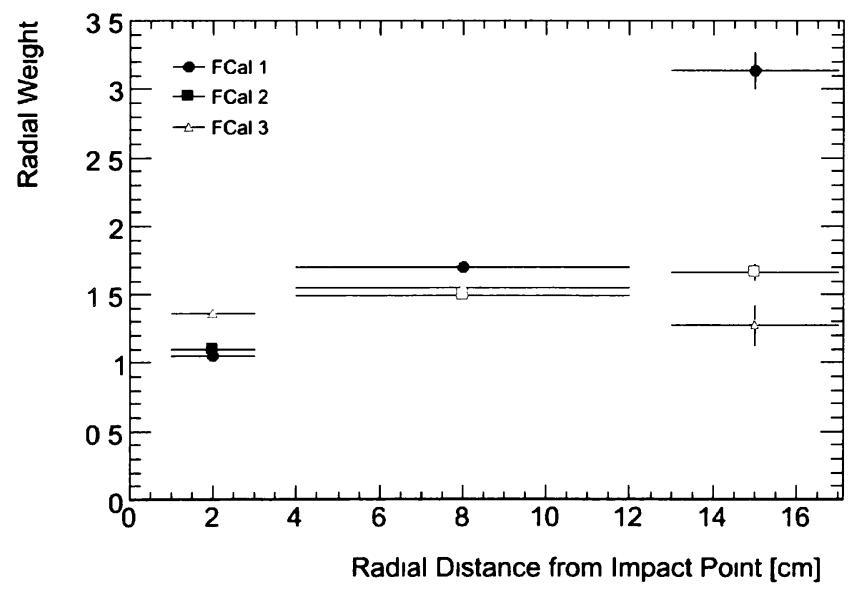

Figure 8.9: Extracted radial weights from $200 \mathrm{GeV} \pi$ data at point $4 \mathrm{~L}$ based on the simplified setup with 3 weights per module and based on the topological clustering of cells. 


\subsubsection{Radial Weights: Simplified Setup Without Tracking}

Finally, a variation on the simplified radial weighting scheme is presented which, in terms of the number of weights assigned per FCal module, does not differ from the simplified setup outlined in the previous section. The crucial difference for this third scheme is the way in which the impact position is defined. Up to this point the tracking information from the BPCs has been used to extrapolate and pinpoint the particle's projected impact position on the front face of each FCal module.

In the real ATLAS environment, of course, though the inner portion of the detector has the ability to determine tracks for charged particles in the pseudorapidity range $|\eta|<2.5$, one would like to be able to determine the position of an event based on calorimeter information alone [4]. The topological clustering algorithm provides an $(x, y, z)$ position for each cluster for a particular event and it is this information which was used in the third and final scheme. In this way, the energy deposits are separated into radial bins as before, however the centre of the concentric radial bins for a given module is determined using only the information from the clusters created by means of the topological clustering algorithm.

In what follows, the projected impact position on the surface of the $i^{\text {th }}$ FCal module will be determined based only on the cluster information provided, and will be written in terms of an $x$ - and $y$-coordinate as $\left(x_{\imath}, y_{\imath}\right)$. This position is then used as the centre of the radial binning, at which point the same process is continued as in the case of the simplified setup using the BPC tracking information. For comparison, the position $\left(x_{\imath}^{\mathrm{BPC}}, y_{\imath}^{\mathrm{BPC}}\right)$ will refer to the projected impact position on the front surface of the $i^{\text {th }}$ 
FCal module as used in the preceding scheme - using the tracking information. Figure 8.10 illustrates the relevant quantities to be introduced in the expressions to follow.

Suppose one were to label the weighted cluster centre position as $\left(x_{c}, y_{c}, z_{c}\right)$ in the ATLAS coordinate system. It is important to note that this weighted position is calculated based on the energy cluster or clusters for a particular event and will not, in general, be situated near the surface of either of the modules. It is for this reason that its position is written in terms of all three cartesian coordinates.

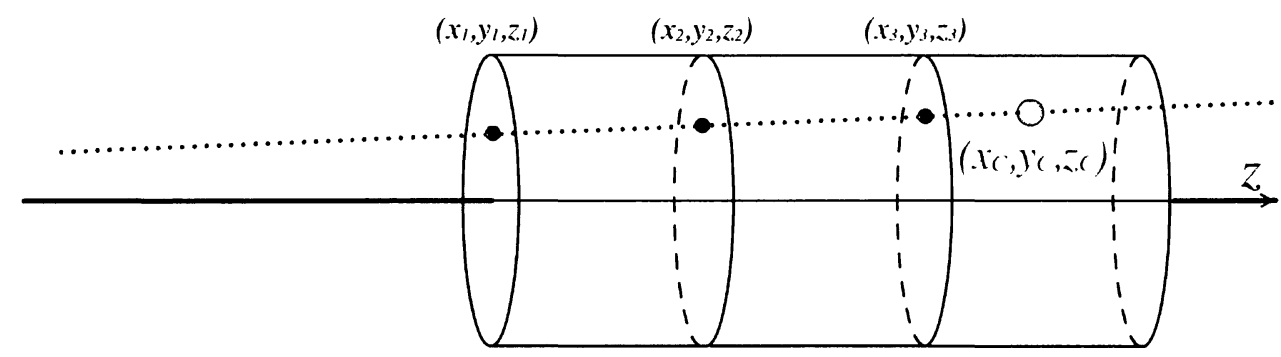

Figure 8.10: A representation of the FCal modules in the ATLAS coordinate system, illustrating the particular FCal geometry and pertinent variables when determining the projected cluster centre coordinates on the module front faces. The dotted line corresponds to the projected path of the particle and is exaggerated in order to illustrate the varying projected impact positions on the different FCal modules.

The radial separation, $r_{c}$, of such a cluster's centre from the centre of the LHC beam pipe in the $x-y$ plane, is given by:

$$
r_{c}=\sqrt{x_{c}^{2}+y_{c}^{2}}
$$

The angle, $\theta$, of the trajectory of the particle associated with a particular cluster, 
assuming that the particle initially travelled from the ATLAS interaction point ${ }^{5}$, satisfies the following:

$$
\tan \theta=\frac{r_{c}}{z_{c}}
$$

Since the cluster's $z$ position can in general be situated at any position between the front face of FCal1 and the rear face of FCal3, as stated previously, one is able to extrapolate, based on the angle $\theta$, the particle's impact position on the front face of each module. For FCal1, assuming the front face is situated at $z_{1}$ and that the impact position's $x$ - and $y$-coordinates are represented by $x_{1}$, and $y_{1}$, respectively, it follows that:

$$
\begin{aligned}
& x_{1}=r_{1} \cdot \cos \phi \\
& y_{1}=r_{1} \cdot \sin \phi
\end{aligned}
$$

where

$$
\tan \phi=\frac{y_{c}}{x_{c}}
$$

and the value $r_{1}$ is given by:

$$
r_{1}=z_{1} \cdot \tan \theta
$$

The third method from this point onwards then extracts radial weights in a similar

\footnotetext{
${ }^{5}$ It should be noted that this was a choice and that in reality, the angle of incidence of test beam particles was roughly fixed as that given in Table 4.1 for the beam point in question. This angle could have alternatively been used in determining the projected positions. A third alternative, which was shown to result unsurprisingly in a greater discrepancy from the projected BPC position, would be to assume a common impact position $\left(x_{c}, y_{c}\right)$ for the front surface of all three modules, thereby assuming the particles to be travelling in the positive $z$-direction.
} 
fashion to the simplified radial weight setup with the main difference being that no BPC tracking information was used in the process.

It is illustrative to show the difference between the projected impact positions on FCal 1 with and without the tracking, namely the difference between the position as determined by the BPC tracking information and that determined by the cluster centre information and the subsequent transformations shown in Equations (8.3) through (8.7). Figure 8.11 specifically shows the absolute differences in both the $x$ - and $y$-directions, written as $\Delta_{x}$ and $\Delta_{y}$, respectively, where:

$$
\Delta_{x}=\left|x_{1}-x_{1}^{\mathrm{BPC}}\right|
$$

and

$$
\Delta_{y}=\left|y_{1}-y_{1}^{\mathrm{BPC}}\right|
$$

The reason that the peaks of the distributions in Figure 8.11 are not situated at zero is due to the pulling of cluster centres to the readout channel centres.

The extracted weight values based on this method without tracking information, for the case of $200 \mathrm{GeV}$ pions at $4 \mathrm{~L}$, are shown in Figure 8.12. It should be noted that this is only done with data based on the topological clustering algorithm since it is only for this case, and not the case of cylindrical clustering, that the relevant cluster information is available. As can be seen in the figure, the weights determined in this way do not vary greatly as compared with those for which the tracking information is used. These weights will subsequently be applied in the following chapter only to those data for which 


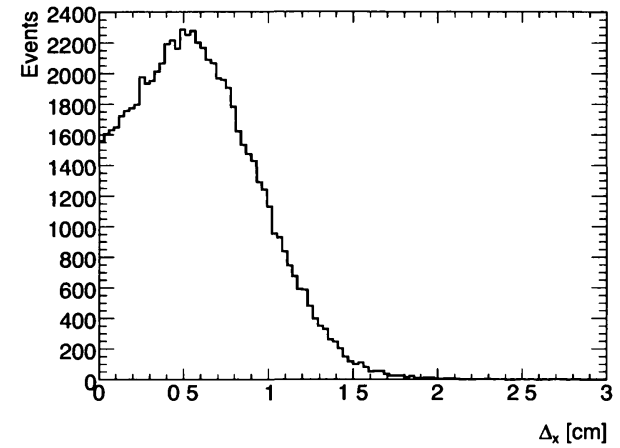

(a) The absolute value of the difference in the projected $x$-position as determined by the BPC tracking information and the weighted cluster centre.

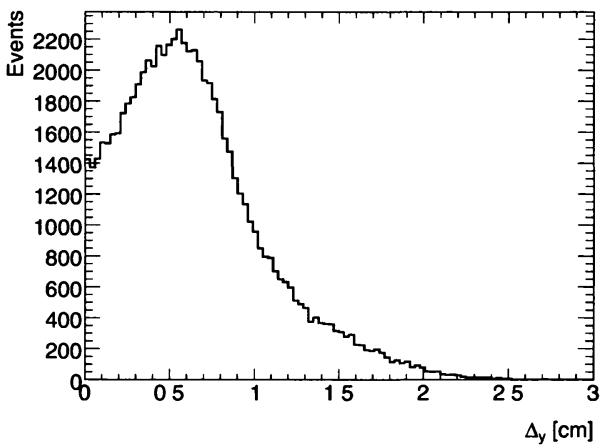

(b) The absolute value of the difference in the projected $y$-position as determined by the BPC tracking information and the weighted cluster centre.

Figure 8.11: The difference in the projected pion impact position on FCal 1 for $200 \mathrm{GeV}$ pion data at $4 \mathrm{~L}$, shown for both the a) $x$ and b) $y$ coordinates.

tracking information was not used.

Finally it should be pointed out that an individual pion event will often involve more than one energy cluster. There are a variety of reasons for the generation of additional clusters, for which a representation is illustrated in Figure 8.13. While for some events additional clusters are to be expected due, for example, to the particular way in which the hadronic shower developed, in other cases their presence could be indicative of the presence of a particularly noisy readout channel. For the 2003 test beam data, a large number of pion events $d \imath d$ involve only one cluster, though for a non-negligible number of events multiple clusters were generated. Due to the way in which the weighted cluster centre position was calculated for each particular event, this resulted, at times, in a projected impact position which differed from the BPC projected position by a substantial amount. It should be recognized, therefore, that not all events are represented in the 


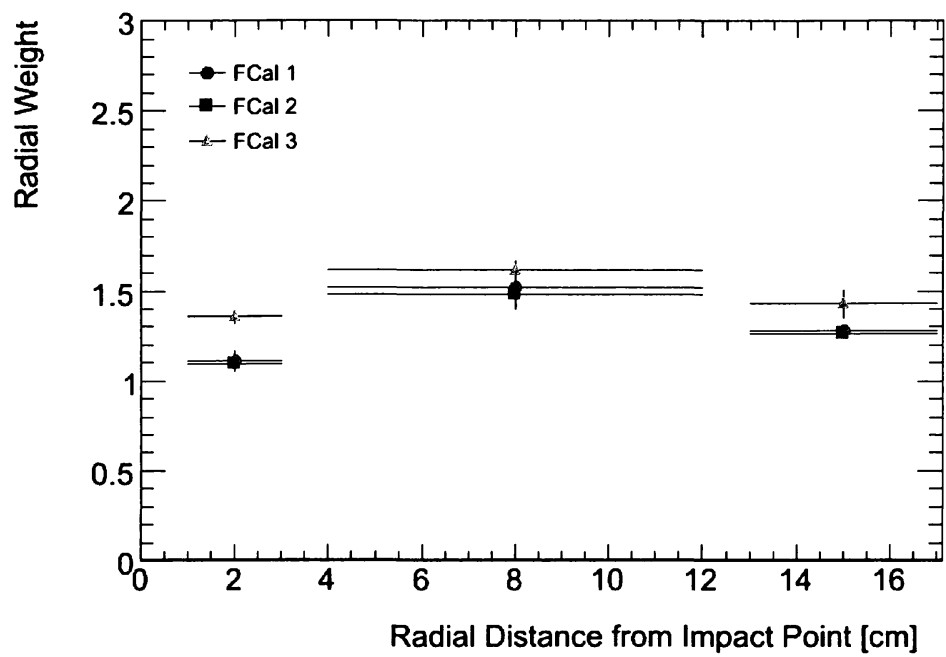

Figure 8.12: Extracted radial weights from $200 \mathrm{GeV} \pi$ data at beam impact point $4 \mathrm{~L}$ based on the simplified setup with 3 weights per module and without the use of BPC tracking information. The cluster centre information is used in its place to determine the reference point for the radial binning. The weights are shown for each FCal module individually as a function of radial distance from the impact point for that module, based on the topological clustering of cells. The error bars include both statistical and systematic errors as explained in the previous section.

distributions shown in Figure 8.11; for a relatively small number of events, the values of $\Delta_{x}$ and $\Delta_{y}$ were significantly larger. No cut was made on the number of clusters in events, as to do this would require a systematic study of the cluster splitting and merging algorithm that was beyond the scope of this thesis.

In a real ATLAS environment, there will of course be many clusters, and at the time of writing cluster identification continues to be an important topic within the ATLAS calorimetry community in terms of understanding the way in which to optimally group 


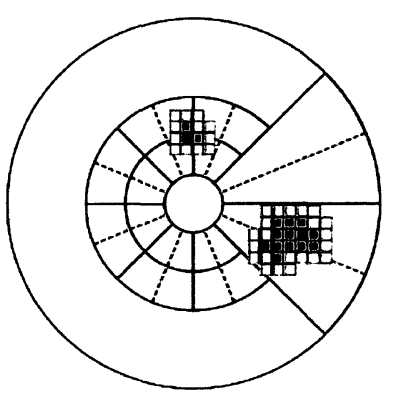

Figure 8.13: A representation of more than one topological cluster being generated based on the clustering algorithm in the forward calorimeter. The physical separation between is meant only to highlight the presence of distinct clusters.

together different energy clusters corresponding to the same particle or jet.

It is important in this case, however, only to show that it is possible to extract weights without specifically using the BPC tracking information so that, at least in principle, it could be done in an ATLAS environment with no tracking information. Moreover, if good agreement can be demonstrated between the results with and without BPC tracking information, this provides confidence in the ability to apply a radial weighting scheme to the real ATLAS environment where tracking information is not available. 


\subsection{Summary of Radial Weights}

In summarizing the radial weighting schemes presented in the current chapter, it should be recalled that even in the case of the modular weight setup, a variety of choices had been considered: the choice of beam impact point $(1,2,3,4 \mathrm{H}$ or $4 \mathrm{~L})$, beam energy value, and clustering algorithm, namely cylindrical or topological. Such choices remain for the case of the radial hadronic weights, with the additional choice of the radial schemes; the standard scheme with 16 weights per module, and the simplified scheme with three weights per module. The latter is then further broken down into the cases with or without the use of tracking information. Clearly the number of possible combinations from which to choose has increased significantly. In order to keep the results clear, the following chapter will continue to focus on beam point $4 \mathrm{~L}$ since, as previously mentioned, the results from this beam point represent the intrinsic response of the forward calorimeter system. Additional plots will nonetheless continue to be presented in Appendix A. 


\section{Chapter 9}

\section{Radial Hadronic Weight Results}

\subsection{Application of Derived Radial Weights}

The results obtained by applying the various radial hadronic weights are now summarized and, where possible, compared to the results shown in Chapter 7 as well as the results from previous analyses involving pion data from the 2003 ATLAS forward calorimeter test beam [12] [13].

\subsection{Reconstructed Energy}

\subsubsection{Cylindrical Clustering Reconstructed Energy Results}

The linearity plot for $4 \mathrm{~L}$ data using radial weights, along with a comparison to previous results, is shown in Figure 9.1 for cylindrical clustering. The version of the radial weighting scheme using cluster centre information as opposed to BPC tracking information can 
only be used in the case of topological clustering and is therefore not included in this plot.

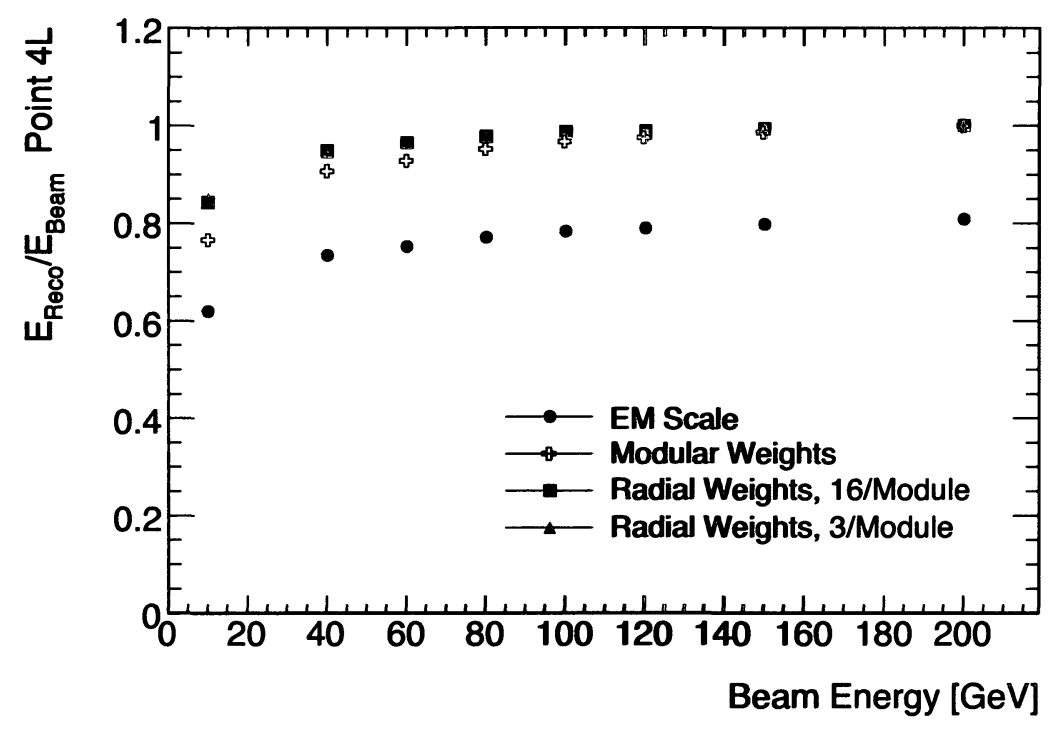

Figure 9.1: Linearity plot for 4L comparing all important weighting schemes for the case of cylindrical clustering. At higher energies, the results from all weighting schemes are nearly coincident and cannot be distinguished from one another, though their response is of course higher than the EM scale values shown in blue. The two radial weighting schemes can be seen to produce nearly identical results.

One can note that at higher energies, all of the weighting schemes investigated return reconstructed energies very close to the beam energy values. The noise-subtracted energy resolution values for each of the schemes must therefore be compared in order to determine the optimal weighting scheme, as will be done in the following section. A marginal improvement in the response over the full range of beam energies can be seen for the results employing radial weight as compared with those employing modular weights, and 
this is particularly noticeable at lower energies.

\subsubsection{Topological Clustering Reconstructed Energy Results}

Figure 9.2, likewise, shows the pion linearity plot for topological clustering and one can note, as with the results from cylindrical clustering, that the application of radial weights from any of the methods yields an improvement, albeit less apparent, over the modular weight results. The fact that more of the energy is sampled in the case of cylindrical clustering results in a noticeably flatter linearity plot as compared with topological clustering.

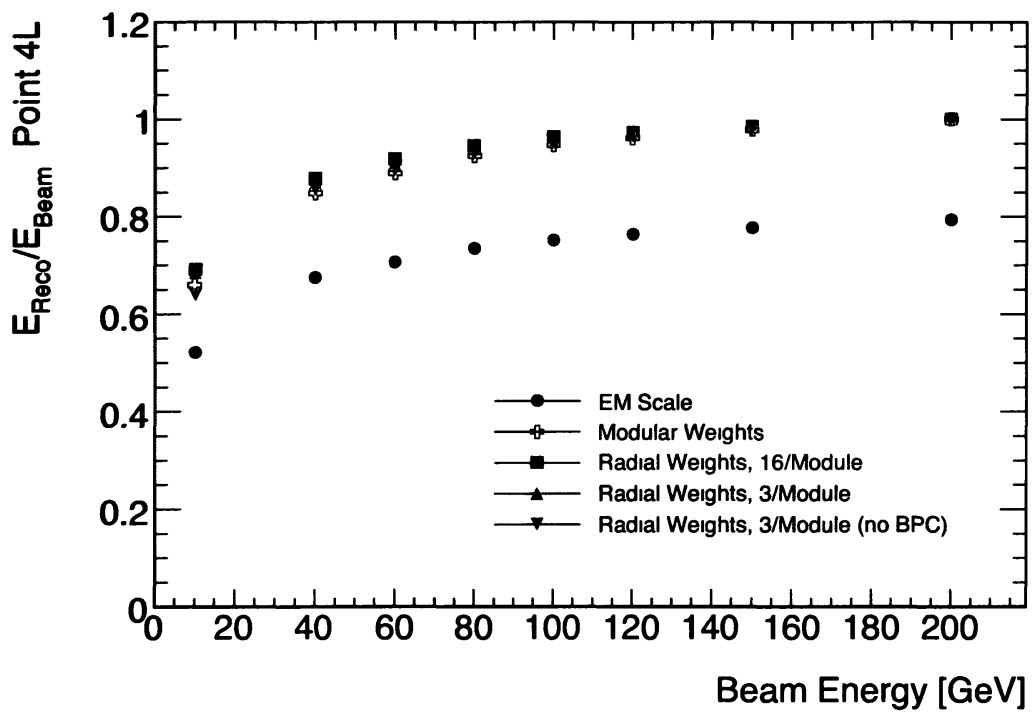

Figure 9.2: Linearity plot for $4 \mathrm{~L}$ comparing all important weighting schemes for the case of topological clustering. As with the case of cylindrical clustering, the response is similar for all weighted data, particularly at higher energy where points appear coincident. 


\subsection{Noise-Subtracted Energy Resolution}

Having noted the similar results from all weighting schemes based on the linearity plots, the resulting noise-subtracted energy resolution plots for the different weighting schemes can be compared. This will be done separately for the two clustering algorithms.

\subsubsection{Cylindrical Clustering Resolution Results}

Figure 9.3 shows the noise-subtracted energy resolution values for the various weighting schemes as a function of beam energy for the case of cylindrical clustering. The previous results based on the modular weighting scheme are nearly coincident with the EM scale results, though a marked improvement in resolution can clearly be observed for the case of radial weighting, for either the case of the standard or simplified scheme. The noisesubtracted energy resolution values for the $200 \mathrm{GeV}$ point are shown in Table 9.1 along with the improvement over the EM scale value for the cases in which hadronic weights were applied.

Table 9.1: Noise-subtracted energy resolution values for $4 \mathrm{~L}$ pion data at $200 \mathrm{GeV}$ for the various weighting schemes presented as well as EM scale results for the cylindrical clustering of cells.

\begin{tabular}{|c|c|c|}
\hline Weighting Scheme & $\sigma_{\mathrm{E}} / \mathrm{E}[\%](200 \mathrm{GeV})$ & Improvement [\%] \\
\hline \hline EM Scale & $9.90 \pm 0.02$ & - \\
\hline Modular & $9.76 \pm 0.02$ & 1.4 \\
\hline Radial (16 weights/module) & $7.06 \pm 0.02$ & 28.7 \\
\hline Radial (3 weights/module) & $6.81 \pm 0.02$ & 31.2 \\
\hline
\end{tabular}




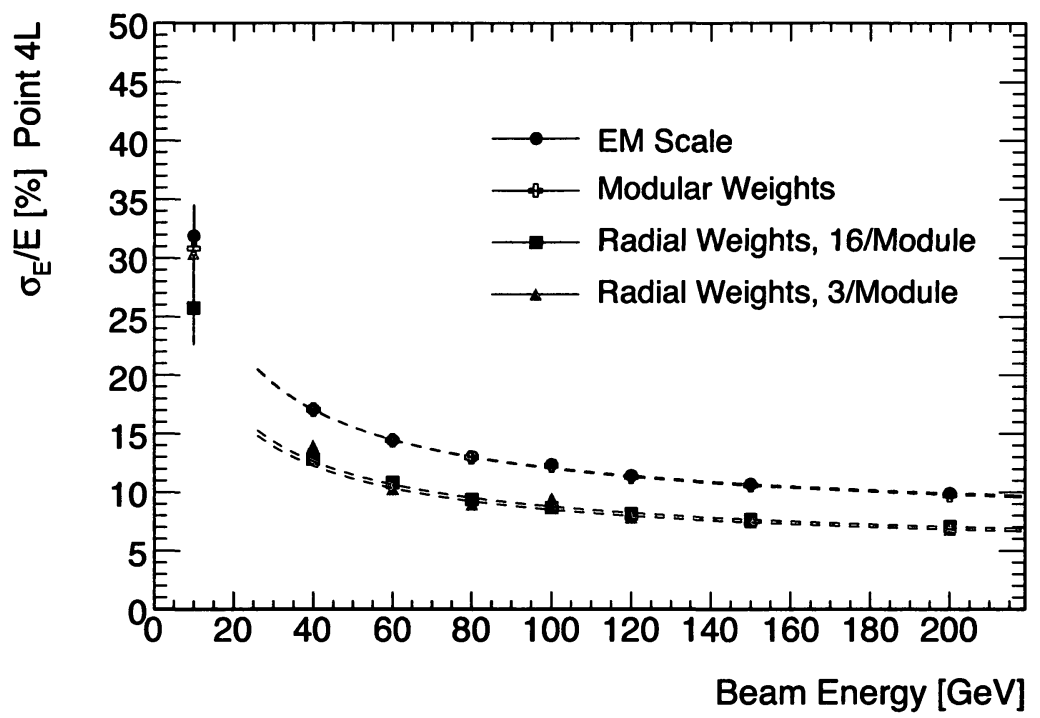

Figure 9.3: Noise-subtracted energy resolution plot for $4 \mathrm{~L}$ comparing all important weighting schemes for the case of cylindrical clustering of cells. The results for modular weights, though difficult to distinguish, are very close in value to and nearly indistinguishable from the EM scale results.

It can be seen that in terms of cylindrical clustering, the lowest value for the noisesubtracted energy resolution is found to be the case when radial weighting with 3 weights per module is used, though it returns a value similar to the case of 16 weights per module. The improvement for either case with respect to the EM scale result is significantly greater than the improvement using modular weights. The results for the stochastic and constant terms based on the two-parameter fit for the noise-subtracted energy resolution are summarized in Table 9.2, where all but the $10 \mathrm{GeV}$ points have been included in the fit, as was the case in Chapter 7. 
Table 9.2: Results for the two-parameter noise-subtracted energy resolution fit for 4L pion data for the various weighting schemes presented in Chapter 8 as well as the modular weighting scheme and the EM scale. Results shown are for the cylindrical clustering of cells. Also included for comparison are the results based from the 2008 publication of an analysis of FCal test beam pion data. Note that in this case, systematic errors have been included.

\begin{tabular}{|c|c|c|}
\hline Weighting Scheme & $a\left[\% \cdot \mathrm{GeV}^{\frac{1}{2}}\right]$ & $b[\%]$ \\
\hline \hline EM Scale & $97.8 \pm 0.6$ & $7.1 \pm 0.1$ \\
\hline Modular & $98.7 \pm 0.6$ & $6.8 \pm 0.1$ \\
\hline Modular (2008 Paper [13]) & $94.2 \pm 1.6$ & $7.5 \pm 0.4$ \\
\hline Radial (16 weights/module) & $74.1 \pm 0.5$ & $4.7 \pm 0.1$ \\
\hline Radial (3 weights/module) & $72.0 \pm 0.7$ & $4.5 \pm 0.1$ \\
\hline
\end{tabular}

\subsubsection{Topological Clustering Resolution Results}

The noise-subtracted energy resolution values for the case of topological clustering are shown for the various schemes in Figure 9.4, where the results for the additional scheme introduced in Section 8.3.3, which does not make use of the BPC tracking information, have also been included and are represented by the inverted triangular points.

The $200 \mathrm{GeV}$ noise-subtracted energy resolution values, as well as their improvement over the EM scale, are summarized in Table 9.3. As with the cylindrical clustering results, the marginal improvement in the case of the modular weights results in their points being difficult to distinguish from the EM scale points. Both radial weighting schemes are again shown to result in a significant improvement over the EM scale and modular weighting results. When no BPC tracking information is used to determine the centre of the radial 


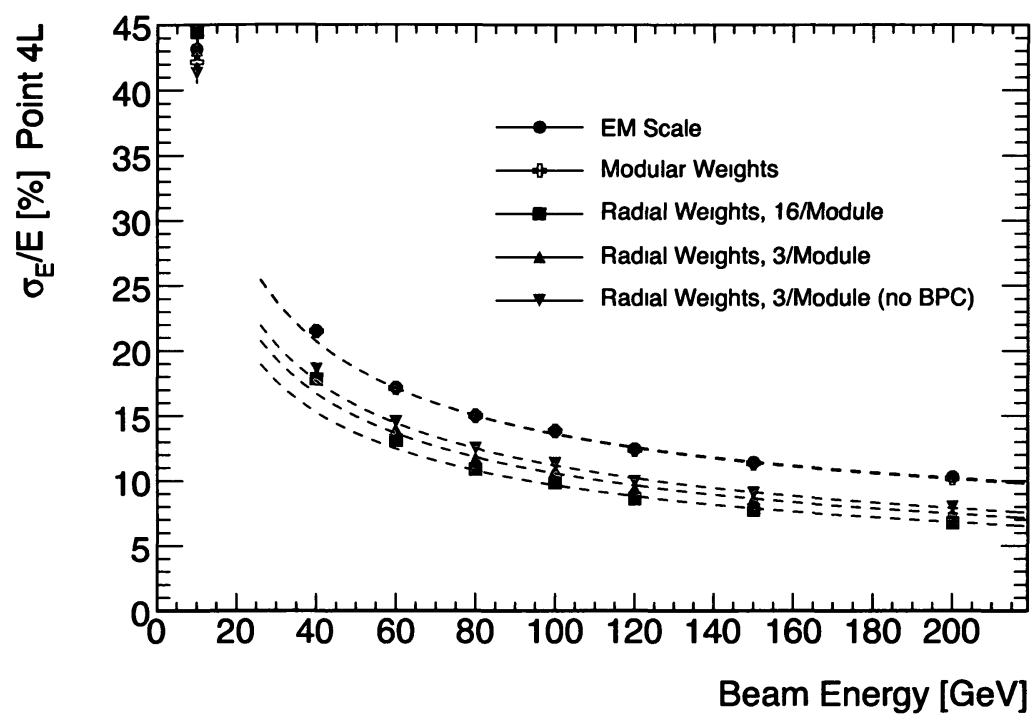

Figure 9.4: Energy resolution plot for 4L comparing all important weighting schemes. The results are shown based on the topological clustering of cells.

bins, the projected impact position resolution is degraded, and one would therefore expect a subsequent broadening of the reconstructed energy distributions. This would result in higher noise-subtracted energy resolution values, which is seen to be the case with the inverted triangular points ${ }^{1}$.

The greatest improvement in energy resolution obtained, based on the $200 \mathrm{GeV}$ data for topological clustering, corresponds to the standard radial weighting scheme in which 16 radial weights are applied per module. The improvement over the EM scale for the $200 \mathrm{GeV}$ point in this case is found to be $34.5 \%$, which is higher than the case of cylindrical clustering, though the EM scale values for each of the clustering algorithms

\footnotetext{
${ }^{1}$ Labelled as Radial Weights, 3/Module (no BPC)
} 
Table 9.3: Noise-subtracted energy resolution values and improvement over the EM scale results for $4 \mathrm{~L}$ pion data for the various weighting schemes presented as well as EM scale results for the topological clustering of cells.

\begin{tabular}{|c|c|c|}
\hline Weighting Scheme & $\sigma_{\mathrm{E}} / \mathrm{E}[\%](200 \mathrm{GeV})$ & Improvement [\%] \\
\hline \hline EM Scale & $10.29 \pm 0.02$ & - \\
\hline Modular & $10.19 \pm 0.02$ & 1.0 \\
\hline Radial (16 weights/module) & $6.74 \pm 0.02$ & 34.5 \\
\hline Radial (3 weights/module) & $7.49 \pm 0.02$ & 27.2 \\
\hline Radial (3 weights/module, no BPC) & $8.82 \pm 0.02$ & 14.3 \\
\hline
\end{tabular}

are recognizably different.

The two-parameter fit results for topological clustering are summarized in Table 9.4. As before, the $10 \mathrm{GeV}$ points have been omitted from the fits. In the case of the radial weighting scheme with 16 weights per module, the stochastic and constant term were found to be:

$$
\frac{\sigma_{E}}{E}=\frac{(96.6 \pm 0.1) \%}{\sqrt{E(G e V)}} \oplus(0.00 \pm 0.12) \%
$$

which do satisfy the ATLAS requirements in the forward region shown in Equation (5.6), though what meaning can be drawn from the constant term - thought to be an artifact of the topological clustering algorithm design - remains uncertain. 
Table 9.4: Results for the two-parameter noise-subtracted energy resolution fits for 4L pion data for the various weighting schemes. Results shown are for the topological clustering of cells.

\begin{tabular}{|c|c|c|}
\hline Weighting Scheme & $a\left[\% \cdot \mathrm{GeV}^{\frac{1}{2}}\right]$ & $b[\%]$ \\
\hline \hline EM Scale & $127.2 \pm 0.5$ & $4.88 \pm 0.09$ \\
\hline Modular & $127.7 \pm 0.5$ & $4.61 \pm 0.09$ \\
\hline Radial (16 weights/module) & $96.6 \pm 0.1$ & $0.00 \pm 0.12$ \\
\hline Radial (3 weights/module) & $105.7 \pm 0.1$ & $0.00 \pm 0.24$ \\
\hline Radial (3 weights/module, no BPC) & $119.3 \pm 0.4$ & $2.15 \pm 0.16$ \\
\hline
\end{tabular}

\subsection{Overall Summary of Weighting Results}

The application of radial weights, which were designed to exploit the lateral properties of hadronic showers resulting from the beams of pions, has been shown to significantly improve the energy resolution at beam point $4 \mathrm{~L}$. The greatest improvement in energy resolution, based on the $200 \mathrm{GeV}$ point, is the case for which the topological clustering of cells is employed and 16 radial weights are both derived and applied. As previously mentioned however, all weighting schemes investigated yield similar results in terms of the linearity plots, where cylindrical clustering offers an improvement over topological clustering in terms of the uniformity of the response for varying beam energies.

Figure 9.5 clearly highlights the effect of applying hadronic weights to pion data at $4 \mathrm{~L}$. The figure corresponds to the data based on cylindrical clustering, though similar plots could be shown for topological clustering. Three reconstructed energy distributions are shown for which a different energy weighting scheme was used for each. The distribution 
to the left is clearly that for which no hadronic weights have been applied - the EM scale distribution. The other two distributions have both been shifted so that the reconstructed energy from the distribution is exactly $200 \mathrm{GeV}$. One can see the clear improvement in energy resolution in the case of the taller, blue peak, for which radial weights have been applied, over the case of the shorter and broader, red distribution, corresponding to the application of modular hadronic weights.

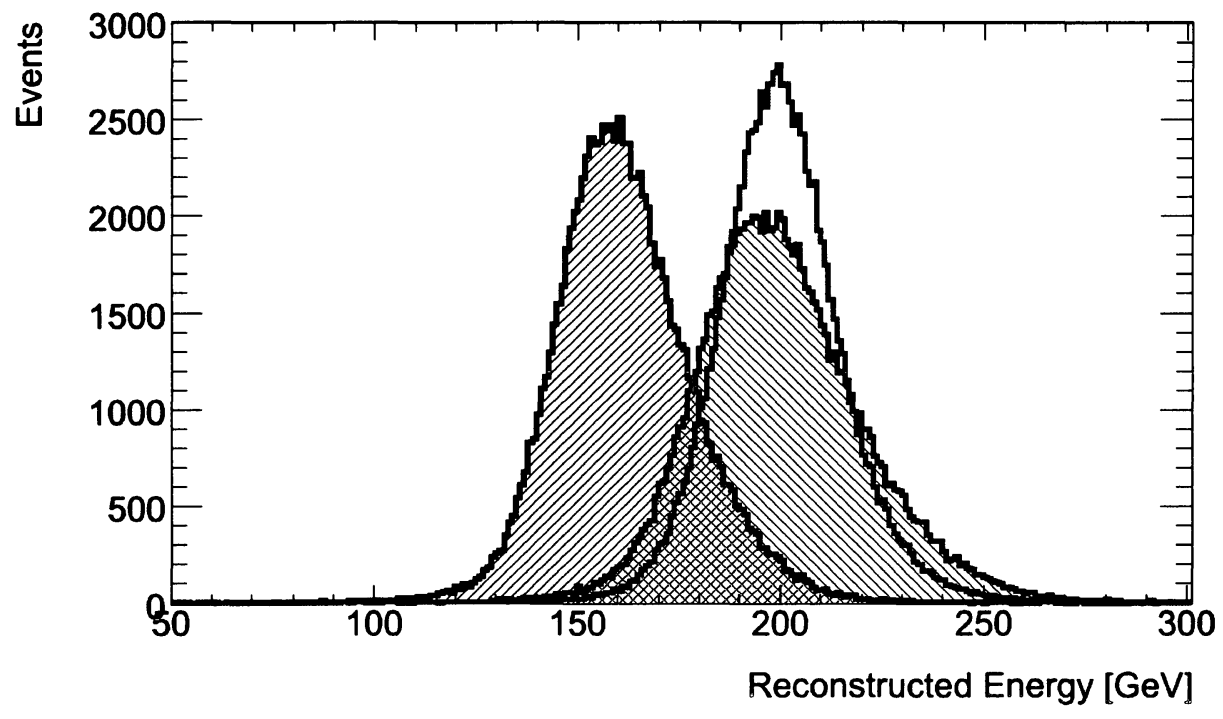

Figure 9.5: Reconstructed energy distributions for all $200 \mathrm{GeV}$ pion data events at 4L. The left-most black cross-filled distribution corresponds to the EM scale results, whereas the other distributions correspond to the result for which hadronic weights have been applied. The shorter and broader distribution with a red cross-filled pattern, as expected, corresponds to the modular weights, with the remaining distribution corresponding to the data with radial weights applied. All distributions correspond to data for which the cylindrical clustering of cells was used.

Based on a comparison of the linearity and noise-subtracted energy resolution values, 
it can be seen from the application of the $200 \mathrm{GeV}$ radial weights that the radial weighting scheme is the optimal choice of the hadronic weighting schemes investigated, and that there is little difference between using the standard or simplified setup. While the cylindrical clustering algorithm yields a more stable and accurate energy response at all beam energy values, based on the application of $200 \mathrm{GeV}$ radial weights, the advantage of the topological algorithm is that it makes use of more noise-suppression in creating energy clusters, and one can use the weighted cluster centre position instead of tracking information, at least in the test beam environment, with only a slightly degraded energy resolution.

Having examined the different weighting schemes, it remains to be seen what the effect of such hadronic weights will be when applied to data from the other beam impact points, particularly those corresponding to so-called high- $\eta$ regions. These regions are of great interest to analyses requiring an accurate measurement of the energy of jets in the forward region, or of missing transverse energy. 


\section{Chapter 10}

\section{Inner Edge Energy Reconstruction}

\subsection{Energy Loss at the Inner Edge of the FCal}

The five beam points chosen for the FCal test beam are shown as a reminder in Figure 10.1. Those three points situated closest to the ATLAS beam point, namely points 1,2 and 3, are said to be inner edge points for obvious reasons.

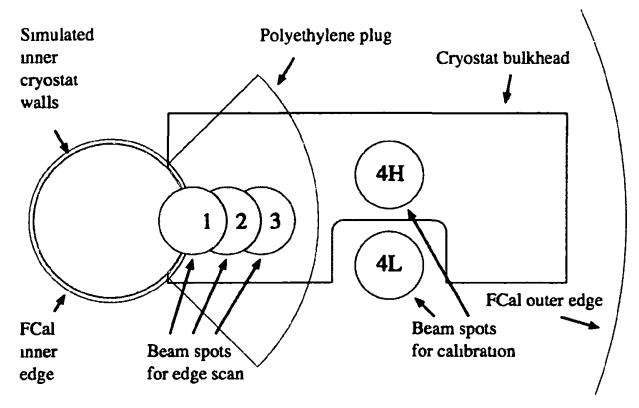

Figure 10.1: The five selected beam points from the 2003 FCal test beam setup. 
It can be expected, given the lateral shapes of hadronic showers as shown in Section 2.5 , that as one approaches the inner area of the FCal corresponding to the location of the beam pipe, shower particles will essentially encounter a vacuum region. One may then naturally wonder what will happen to the reconstructed energy for such cases. Undoubtedly some energy will be accounted for when particles deposit energy in the active cells on the opposite side of the beam pipe, but overall a lower response could be expected due to so-called energy 'lost' down the beam pipe. It is in fact for this very reason that such inner points were selected for the 2003 test beam - a point which was briefly alluded to in Section 4.3.

Having seen the improvement in energy resolution with radial hadronic weights, it was hoped that the application of radial weights to the data at such inner points would help to offset this 'lost' energy effect by weighting more heavily the energy deposited in cells situated at smaller radial separations from the projected beam impact position.

\subsection{Reconstructed Energy Radial Profile}

\subsubsection{Energy Profile at the Electromagnetic Scale}

One can clearly see the lower response at the inner region in Figure 10.2(a), which shows a two-dimensional histogram of the reconstructed energies of pions at the EM scale as a function of the projected radial impact distance from the centre of the beam pipe on the front surface of the first FCal module, referred to as $R 1$. The BPC tracking information was used to obtain the projected positions on the surface of FCal 1. The data from all beam impact points for $200 \mathrm{GeV}$ pion data are shown, with the same standard cuts as 
those outlined in Chapter 4 and based on the topological clustering of cells. The black data points in these two-dimensional histograms correspond to average points.

Such a radial profile shape for the pion reconstructed energy has also been demonstrated by other FCal test beam group members in studies of Monte Carlo data [17]. The independent variable $R 1$ was chosen due to the fact that one generally works in terms of an $x-y$ coordinate system with the forward calorimeters, though those working on general ATLAS analyses not specifically associated with the FCal will tend to look at distributions with respect to $\eta$. For this reason, the same plot to that introduced above but with $\eta$ as the independent variable is included in Appendix A.4 for interest. The gap region in both of these plots is due to the existence of a radial region between points 3 and points $4 \mathrm{H}$ and $4 \mathrm{~L}$ for which no data were collected.

Based on the tracking information, some particles are projected to impact the FCal at a radial distance lower than the inner radius of the FCal module itself as indicated by the black vertical line in Figure 10.2(a). Not surprisingly, the reconstructed energy for such points is significantly lower than near the outer beam points, though one is generally not as interested in such high- $\eta$ regions close to the beam pipe. The reason for any deposited energy at all may be attributed to the presence of upstream material in the test beam setup which would cause the early initiation of a hadronic shower, which would subsequently cause energy to be deposited in active regions of the detector. The region in $\mathrm{R} 1$ between approximately $73 \mathrm{~mm}$ and $100 \mathrm{~mm}$ is, however, of interest. It was hoped that the application of radial hadronic weights to the pion data would help to boost the response for pions projected to impact the detector in this inner region. 


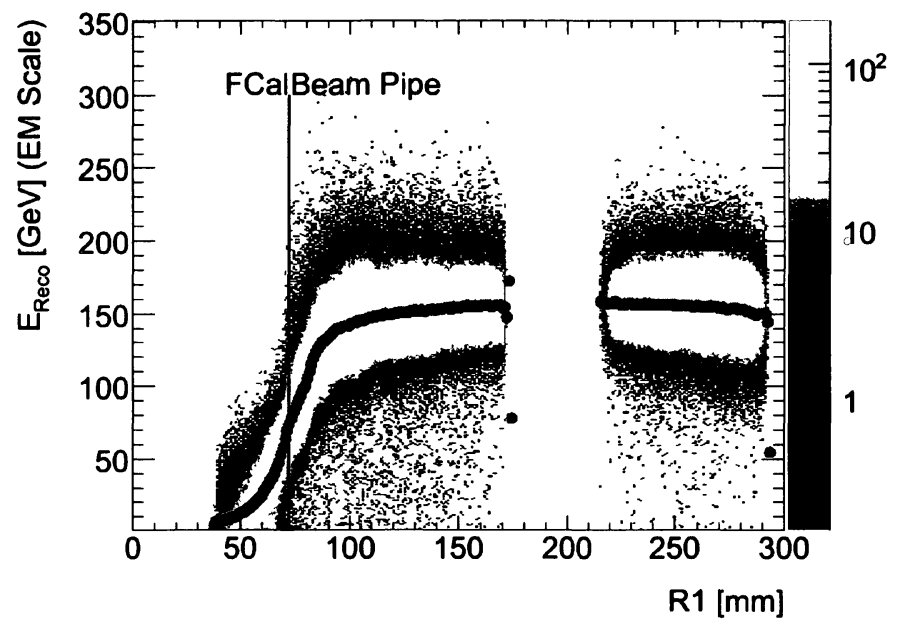

(a) EM scale.

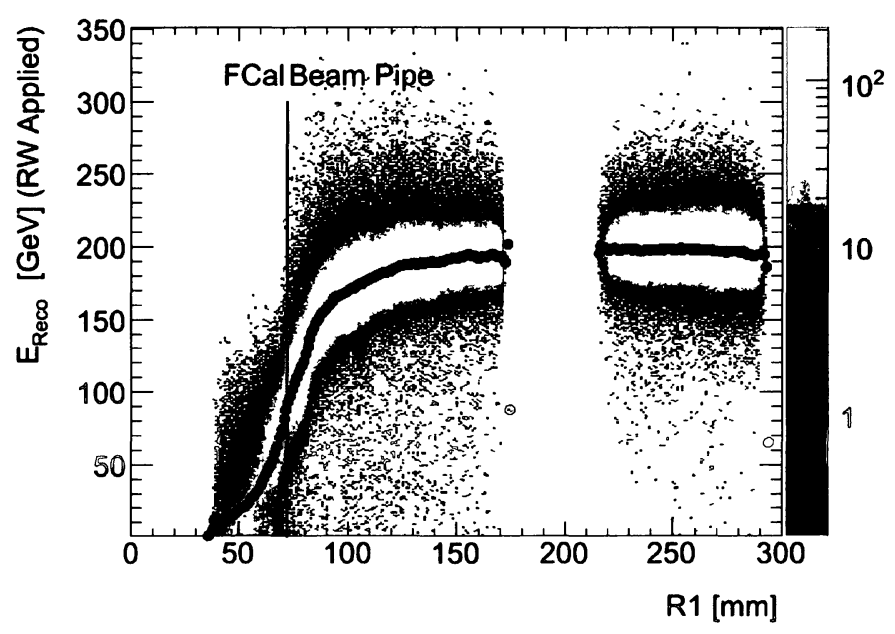

(b) Radial weights applied.

Figure 10.2: Reconstructed energy a) at the EM scale, and b) with the standard setup 4L radial weights applied, shown as a function of projected radial position on the front face of FCal 1. BPC tracking information is used to extrapolate the radial impact position. The black data points corresponding to the average values are shown in addition to the distributions. 


\subsubsection{Energy Profile with Radial Weight Application}

Similar distributions are shown in Figure 10.2(b), with radial weights applied which were derived from point $4 \mathrm{~L} 200 \mathrm{GeV}$ data.

A variety of additional sets of radial weights were applied to the data, and the average values and particular resulting curves for each case can be compared in Figure 10.3. All weights applied were based on $200 \mathrm{GeV}$ data. The green triangular points correspond to $4 \mathrm{~L}$ radial weights based on the simplified setup with three weights per module, and one can see that similar results are obtained for these weights as those for the case of the standard 16 weight setup, shown as red squares.

As a next step, data from each beam point had radial weights applied which were derived for the same point in question. This had the effect of shifting the plateau region to values of $R 1$ near $110 \mathrm{~mm}$. At radial distances below this however, there exists a crossover region between beam points 1 and 2. The extracted weights for these two points differ greatly, with significantly higher weight values for point 1 as can be seen in Appendix A.3 and the mixture of data from these two points in the same region of $R 1$ act such as to produce average reconstructed energies in some cases above $250 \mathrm{GeV}$.

Finally, point-specific weights were applied to data, with the exception that point 2 weights were applied to both the data from point 1 and point 2 . This was done in recognizing that the weights from point 1 were too high due to bias from events projected to impact FCal 1 inside the inner edge. The results, illustrated by the purple circular points, show the clear improvement in the uniformity of the response at lower values of $R 1$. In summary, this final method returns an average reconstructed energy within 


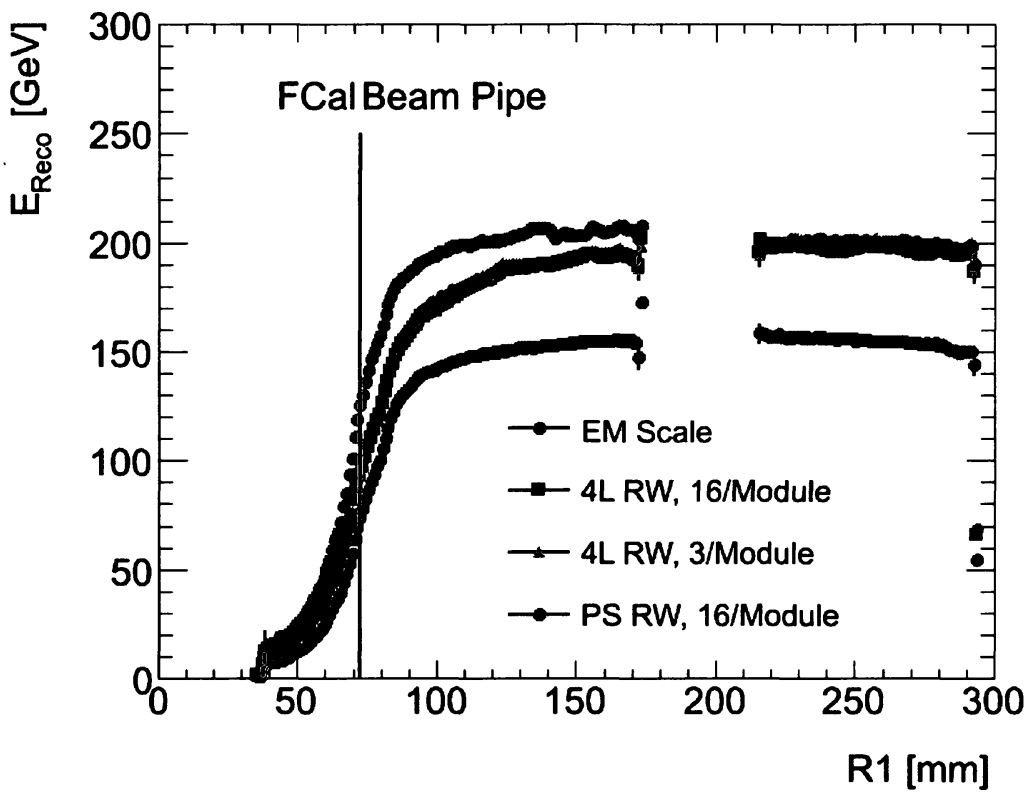

Figure 10.3: Reconstructed energy shown as a function of projected radial position on the front face of FCal 1, determined by the BPC tracking information. The average values from the plots in Figures 10.2(a) and 10.2(b) are shown, in addition to results for which other weights are applied, as outlined in the text. The point-specific (PS) weights, can be seen to yield the most uniform response over the range of $R 1$ values.

roughly $5 \%$ of the beam energy for projected impact positions on the surface of FCal 1 above $90 \mathrm{~mm}$ from the centre of the beam pipe region.

\subsection{Summary of Energy Profiles at the Inner Edge}

The end result of the inner edge study shows that the application of point-specific radial weights can help substantially to extend the plateau region to smaller radial values or, 
equivalently, to higher $\eta$ values, in terms of the reconstructed energy as a function of projected FCal 1 impact position.

An alternate way in which to compensate for the lower energy response in the region closer to the beam pipe would be to fit the data to a particular correction function, and to use the results of such a fit to apply a correction factor to the data. As it was of primary interest only to see if the application of weights would affect the general shape of such profiles however, the application of a correction function is not explored at present, though it has been examined by L. Heelan [17] both for the case of test beam and Monte Carlo data.

One is left to conclude from this study that the application of radial weights to data in the very inner region of the FCal provides a more accurate energy response. An extension to such an analysis could involve fitting the radial weights extracted from the five beam impact points in order to obtain a functional form of radial hadronic weights, specifically as a function of impact position in the forward calorimeter modules. Based on the results in the previous chapter for which no tracking information was used, it would also be possible for such an extension to be investigated by similarly using calorimeter information alone for the impact positions, thereby facilitating such a scheme's incorporation into standard ATLAS analyses. 


\section{Chapter 11}

\section{Conclusion}

The ongoing analysis of data collected as a result of the 2003 ATLAS forward calorimeter test beam is important to the collaboration in terms of helping to understand the signals produced as a result of LHC collisions. As is done with other working groups in the ATLAS collaboration, comparisons between such test beam data and Monte Carlo generated data continue to test and refine the leading physics models in the high-energy regime. Hadronic calibration schemes are currently employed in the analysis of data from the calorimeter system, though such schemes must continually be scrutinized in an effort to find the optimal weighting schemes not only for the system as a whole, but also schemes that are optimized specifically for the forward regions of the ATLAS calorimeters.

Two primary hadronic weighting schemes were investigated based on an analysis of pion data from the forward calorimeter test beam carried out in 2003. These weighting schemes were compared, both with one another, and also to the results obtained through an analysis in which no hadronic weights had been applied and for which data are said 
to have been left at the electromagnetic (EM) scale. Furthermore, two cell clustering schemes, cylindrical clustering and topological clustering were compared, both of which make use of noise reduction by only including cells reasoned to have a significant energy deposit for the particles of interest. Pion data ranging in energy from 10 to $200 \mathrm{GeV}$, and for a series of five beam impact points on the front surface of the FCal modules were used in the analysis. The focus of the comparisons between results was on the point labelled as $4 \mathrm{~L}$, which provides a measure of the intrinsic response of the forward calorimeter.

The primary benchmarks for comparison were the energy response or linearity and the noise-subtracted energy resolution, where a $\chi^{2}$ fit is done in the latter case in order to obtain the values of the two free parameters known as the stochastic and constant terms, common in the field of high-energy calorimetry. Finally, the noise-subtracted energy resolution values are compared between weighted and unweighted data by means of an improvement factor, measured with respect to the EM scale results.

The first hadronic weighting scheme employed a different weight for each of the three forward calorimeter modules, and was referred to as modular hadronic weights. The application of the extracted $200 \mathrm{GeV}$ modular weights to pion data from beam point $4 \mathrm{~L}$ resulted in an energy response within $10 \%$ of the true beam energy for pion energies of $40 \mathrm{GeV}$ and above. Use of the cylindrical clustering algorithm yielded the best results. The improvement in noise-subtracted energy resolution for this scheme with respect to the electromagnetic scale results was found to be $1.4 \%$, and the stochastic and constant terms from the two-parameter fit were given by: 


$$
\frac{\sigma_{E}}{E}=\frac{(98.7 \pm 0.6) \%}{\sqrt{E(G e V)}} \oplus(6.84 \pm 0.06) \%
$$

The second weighting scheme was designed to take advantage of the lateral properties of hadronic showers by applying different weights for different radial positions relative to the shower centre. Such weights were referred to as radial hadronic weights, and were shown to result in an energy response within $5 \%$ of the beam energy for pion energies of $40 \mathrm{GeV}$ and above. In terms of the noise-subtracted energy resolution when cells were clustered based on the topological clustering algorithm, an improvement of $34.5 \%$ was obtained with respect to the electromagnetic scale results. The fit results, which satisfied the ATLAS requirements, were found to be:

$$
\frac{\sigma_{E}}{E}=\frac{(96.6 \pm 0.1) \%}{\sqrt{E(G e V)}} \oplus(0.00 \pm 0.12) \%
$$

Finally the energy response is examined as a function of projected impact position on the front face of the first calorimeter module, in an effort to compensate for the energy lost near the inner edge of the forward calorimeter. The application of radial weights, extracted for each beam impact position individually and subsequently applied to the appropriate data, was found to result in an significantly improved and uniform response in the energy radial profile, particularly close to the inner edge of the forward calorimeter modules.

The application of a radial weighting scheme to ATLAS analyses for the purpose of hadronic calibration in the forward calorimeters has the potential to improve both the calorimeter's energy resolution and performance in the high- $\eta$ region. Further study 
would be required in applying the algorithms presented in this thesis to the $7 \mathrm{TeV}$ collision data currently being collected with the ATLAS experiment. 


\section{Appendix A}

\section{Additional Plots}

\section{A.1 4H Modular Weight Values}

Figures A.1 and A.2 below show the extracted modular weight values for beam impact point $4 \mathrm{H}$, similarly to Figures 6.2 and 6.3 , respectively, which show the weights extracted for beam impact point $4 \mathrm{~L}$. It can be observed that similar results to those for the case of $4 \mathrm{~L}$ were obtained at higher energies. It should be recalled that extra upstream material is present in the case of $4 \mathrm{H}$ which is not present in front of $4 \mathrm{~L}$. As such, the values of the weights for $4 \mathrm{H}$ will need to be higher overall in order to provide more compensation than in the case of $4 \mathrm{~L}$. The higher value for $w_{3}$ in the case of the weights based on the topological clustering algorithm can be observed, similarly to the case for $4 \mathrm{~L}$ in Figure 6.3 . 


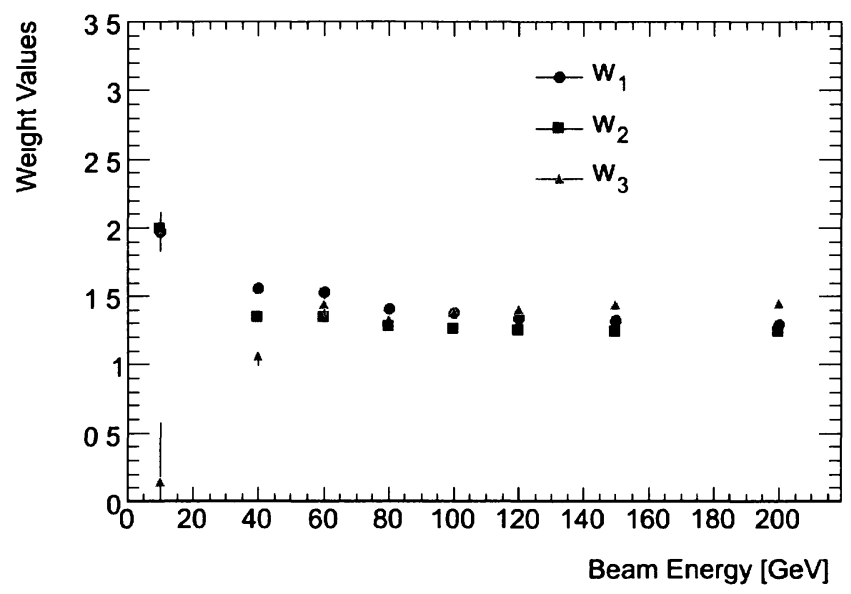

Figure A.1: Extracted modular weights shown as a function of beam energy for beam impact point $4 \mathrm{H}$, where the cylindrical clustering of cells was employed.

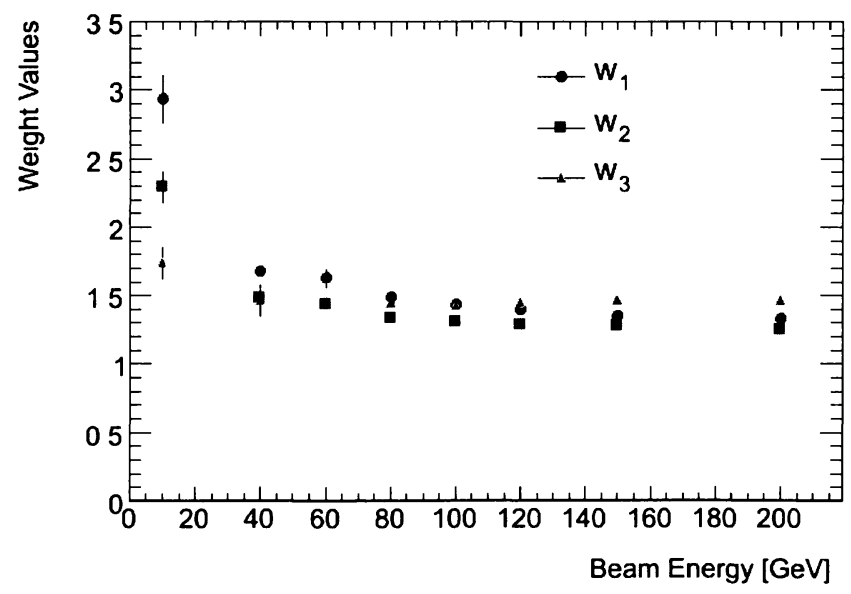

Figure A.2: Extracted modular weights shown as a function of beam energy for beam impact point $4 \mathrm{H}$, where the topological clustering of cells was employed. 


\section{A.2 Modular Weight Results}

Figure A.3 shows the reconstructed energy distributions where no clustering of cells has been used - all cells included during the collection of test beam data ${ }^{1}$ are included. Modular weights have been applied as is apparent in the linearity plot in the lower right of the figure. The $200 \mathrm{GeV}$ weights were derived without the use of a clustering algorithm.

Figure A.4 shows the noise distributions also for the case of no cell clustering algorithm. The same modular weights have been applied as with Figure A.3.

\footnotetext{
${ }^{1}$ Refer to Section 5.1.1
} 

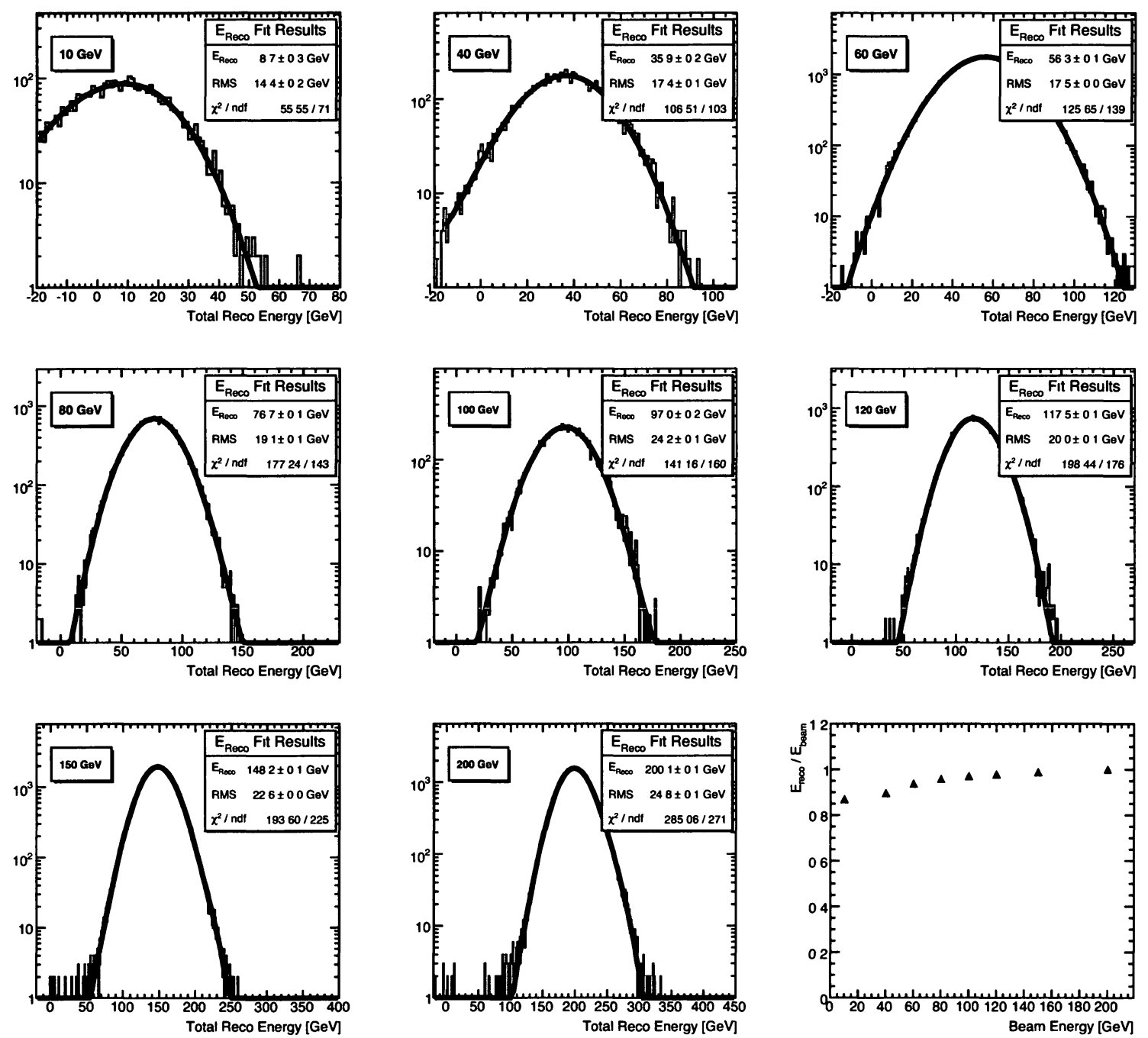

Figure A.3: Distributions of pion reconstructed energy values for all $4 \mathrm{~L}$ events which passed the cuts as summarized in Chapter 4. No clustering of cells - neither cylindrical nor topological clustering - is employed. 

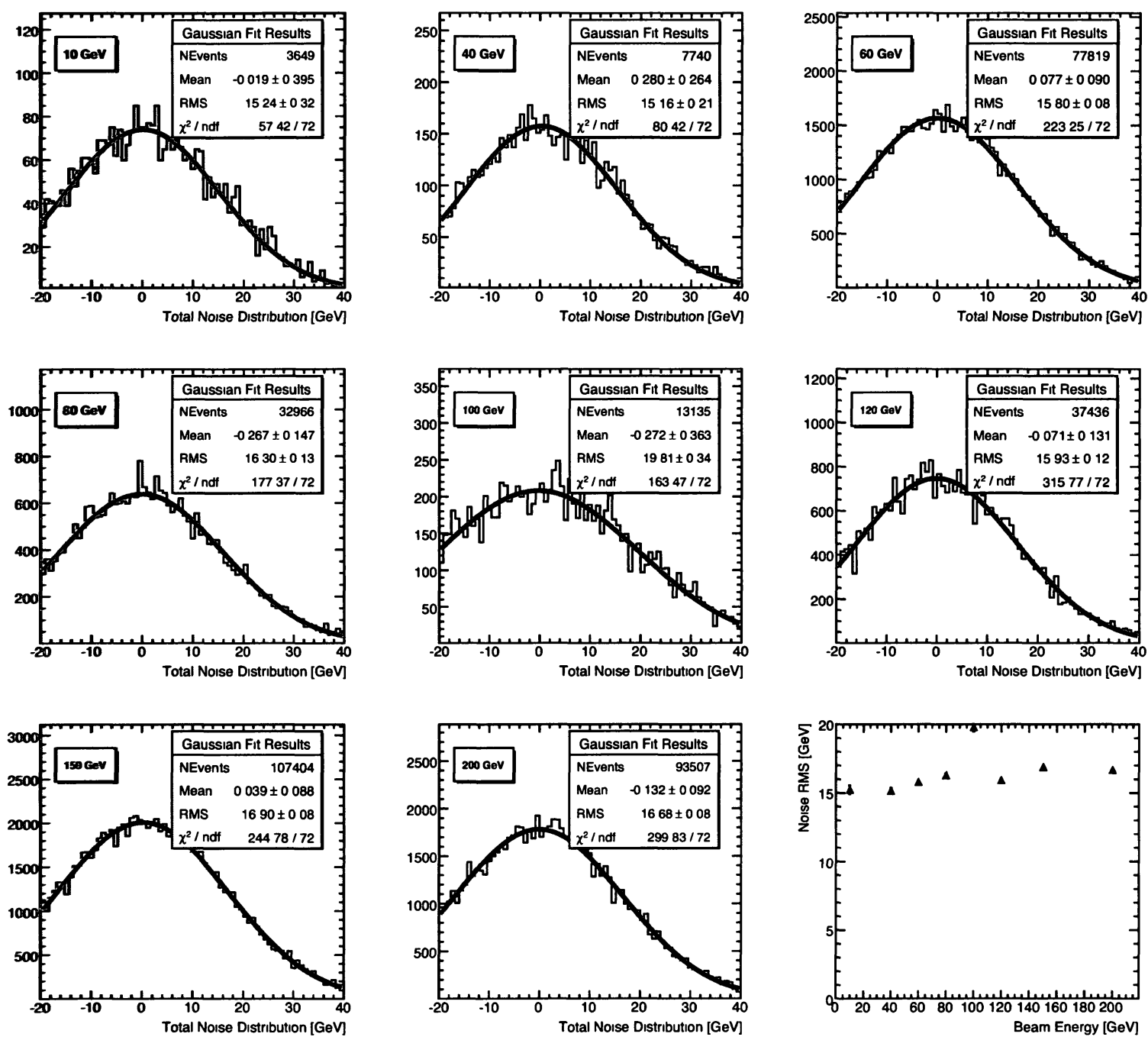

Figure A.4: Noise distributions for $4 \mathrm{~L}$ pion events passing the cuts outlined in Chapter 4 with no cell clustering algorithms employed, having applied the corresponding $200 \mathrm{GeV}$ modular weights derived for the same setup. 


\section{A.3 Radial Weight Values}

The figures below show the radial weights extracted for $200 \mathrm{GeV}$ pion data for beam impact points 1, 2, 3 and $4 \mathrm{H}$.

Figures A.5 through A.10 refer to the radial weights extracted based on the standard setup. Figures A.11 through A.14 correspond to the simplified setup.

It should be noted that the errors included in the figures correspond to those outlined in the text in Section 6.3. 


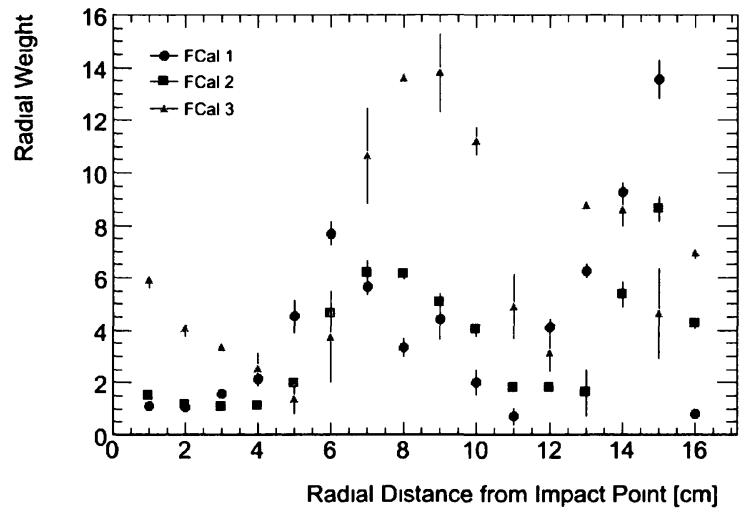

Figure A.5: Point 1: Extracted radial weights from $200 \mathrm{GeV} \pi$ data based on the standard setup with 16 weights per module. The weights are shown for each FCal module individually as a function of radial distance from the impact point for that module, based on the topological clustering of cells.

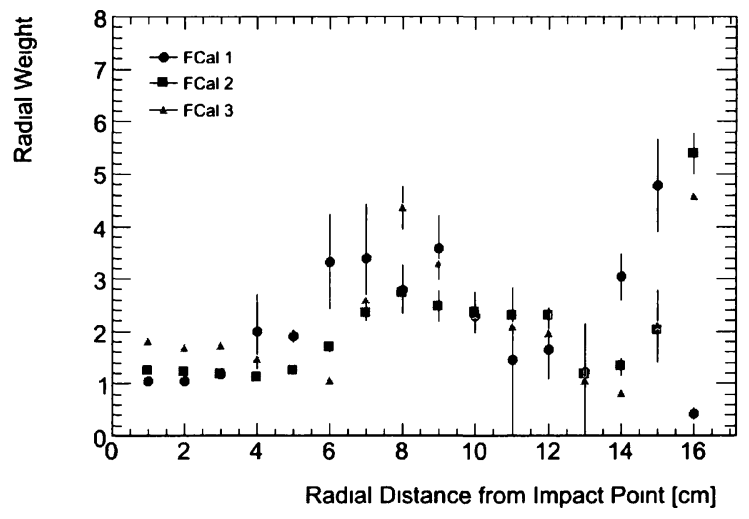

Figure A.6: Point 2: Extracted radial weights from $200 \mathrm{GeV} \pi$ data based on the standard setup with 16 weights per module. The weights are shown for each FCal module individually as a function of radial distance from the impact point for that module, based on the topological clustering of cells. 


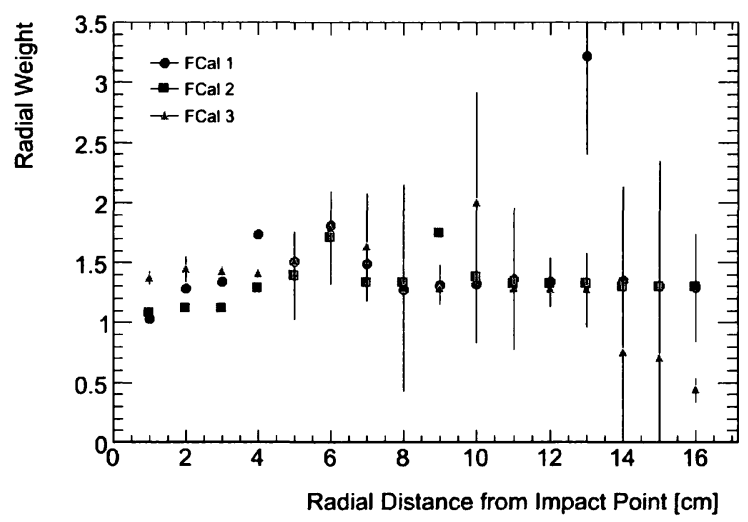

Figure A.7: Point 3: Extracted radial weights from $200 \mathrm{GeV} \pi$ data based on the standard setup with 16 weights per module. The weights are shown for each FCal module individually as a function of radial distance from the impact point for that module, based on the cylindrical clustering of cells.

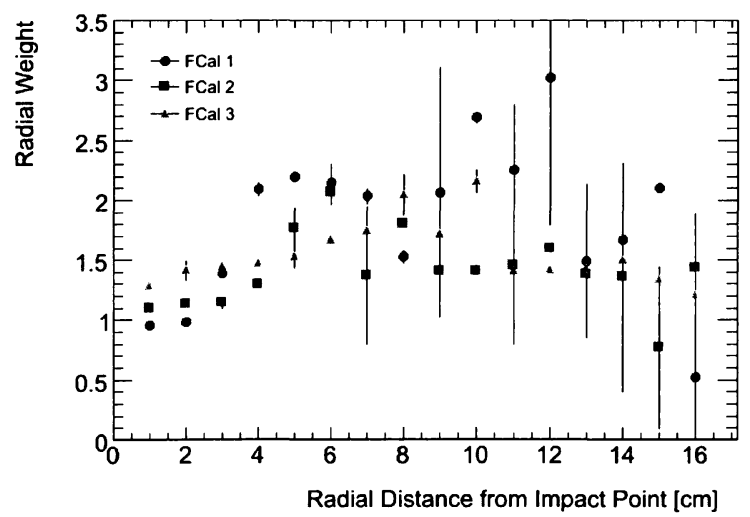

Figure A.8: Point 3: Extracted radial weights from $200 \mathrm{GeV} \pi$ data based on the standard setup with 16 weights per module. The weights are shown for each FCal module individually as a function of radial distance from the impact point for that module, based on the topological clustering of cells. 


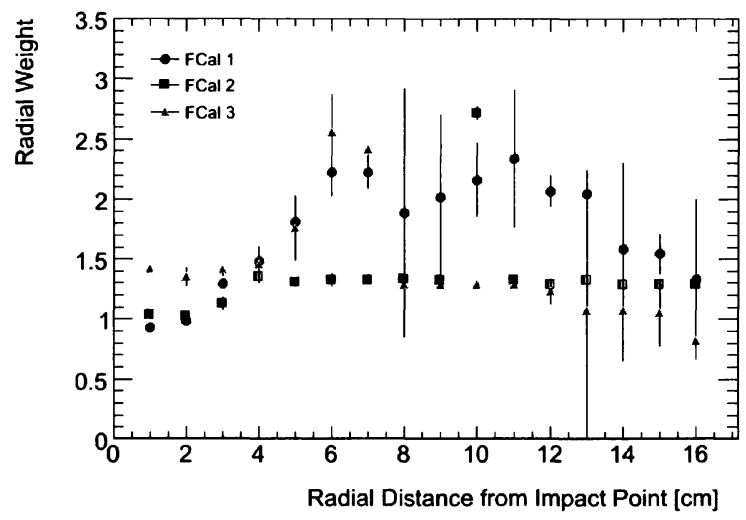

Figure A.9: Point 4H: Extracted radial weights from $200 \mathrm{GeV} \pi$ data based on the standard setup with 16 weights per module. The weights are shown for each FCal module individually as a function of radial distance from the impact point for that module, based on the cylindrical clustering of cells.

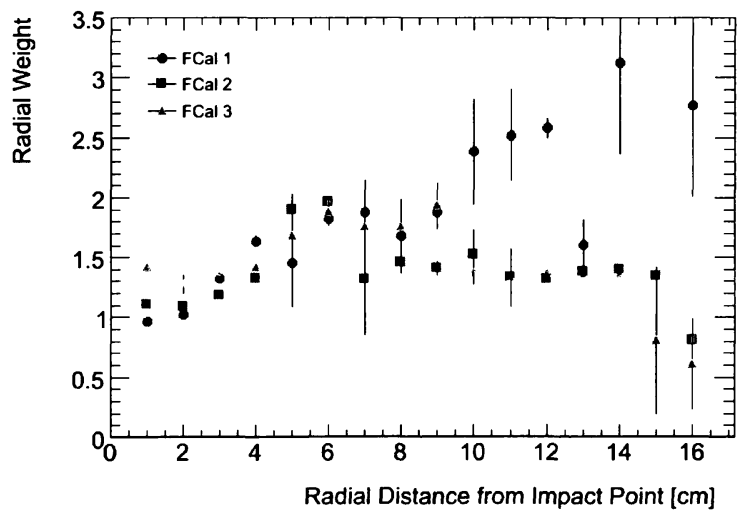

Figure A.10: Point 4H: Extracted radial weights from $200 \mathrm{GeV} \pi$ data based on the standard setup with 16 weights per module. The weights are shown for each FCal module individually as a function of radial distance from the impact point for that module, based on the topological clustering of cells. 


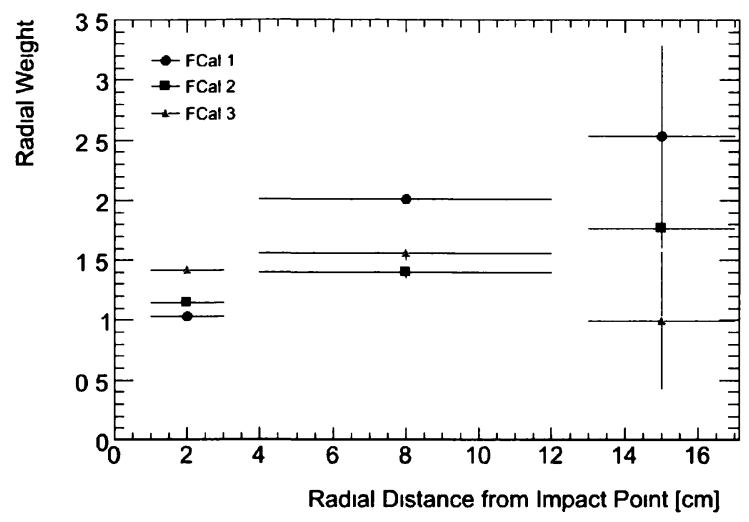

Figure A.11: Point 3: Extracted radial weights from $200 \mathrm{GeV} \pi$ data based on the simplified setup with 3 weights per module. The weights are shown for each FCal module individually as a function of radial distance from the impact point for that module, based on the cylindrical clustering of cells.

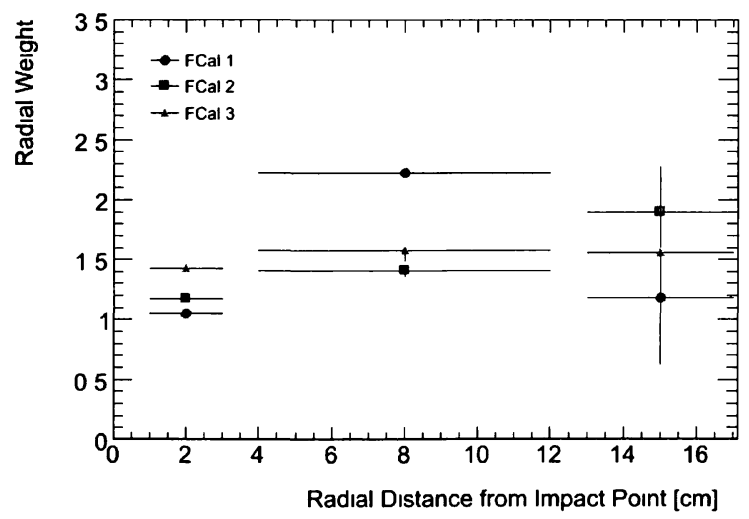

Figure A.12: Point 3: Extracted radial weights from $200 \mathrm{GeV} \pi$ data based on the simplified setup with 3 weights per module. The weights are shown for each FCal module individually as a function of radial distance from the impact point for that module, based on the topological clustering of cells. 


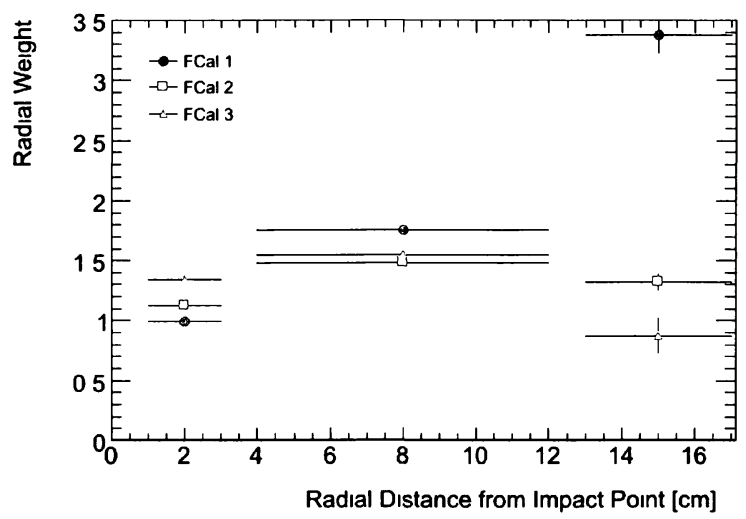

Figure A.13: Point 4H: Extracted radial weights from $200 \mathrm{GeV} \pi$ data based on the simplified setup with 3 weights per module. The weights are shown for each FCal module individually as a function of radial distance from the impact point for that module, based on the cylindrical clustering of cells.

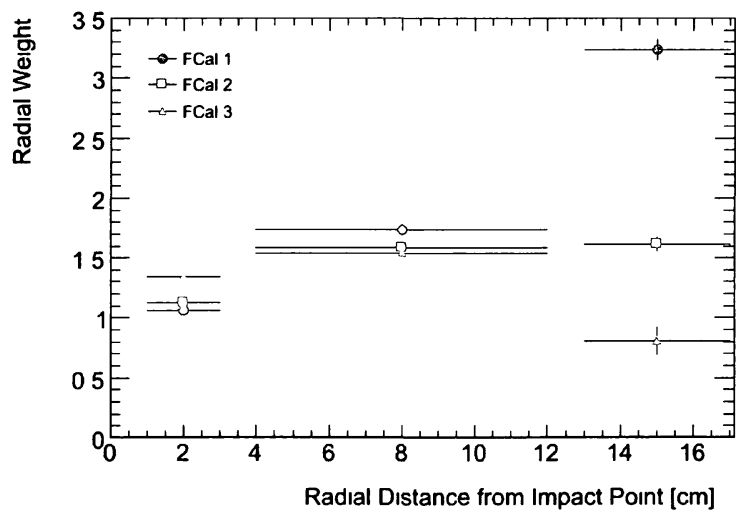

Figure A.14: Point 4H: Extracted radial weights from $200 \mathrm{GeV} \pi$ data based on the simplified setup with 3 weights per module. The weights are shown for each FCal module individually as a function of radial distance from the impact point for that module, based on the topological clustering of cells. 


\section{A.4 Reconstructed Energy Profiles}

The comparison of reconstructed energy distributions are shown as a function of $\eta$ in Figure A.15 just as was shown as a function of R1 in Figure 10.3. One can note the loss of energy due to the proximity of the LHC beam pipe at values of $\eta$ above 4.4.

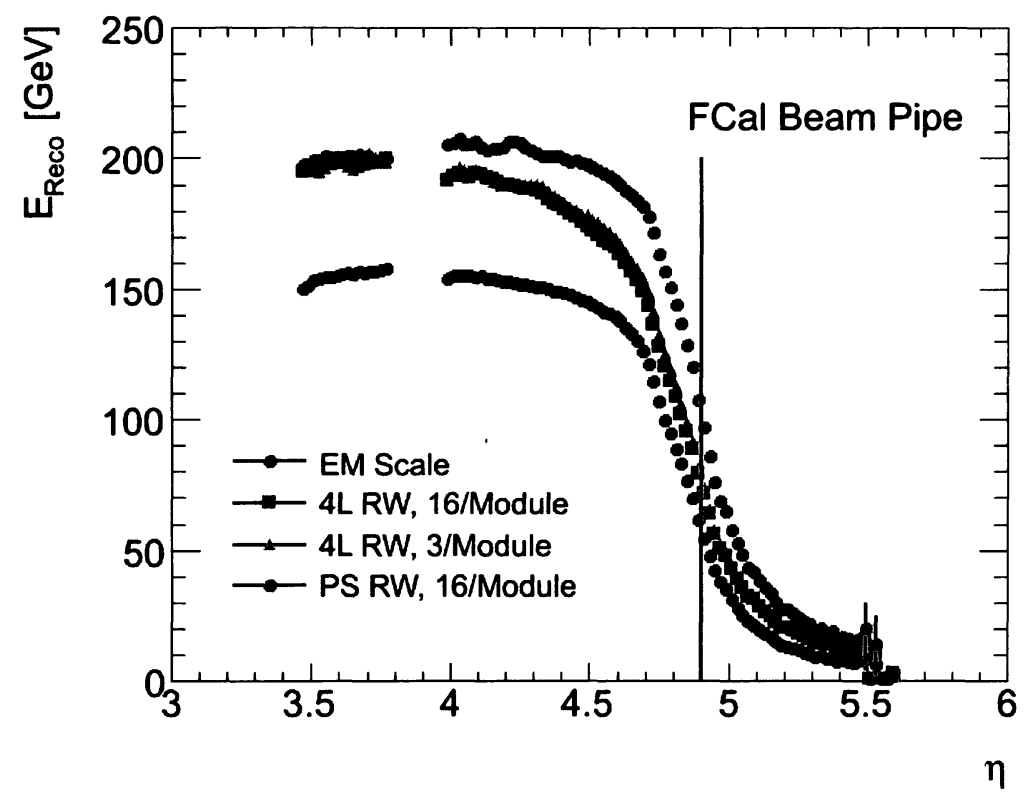

Figure A.15: Reconstructed energy shown as a function of pseudorapidity based on the trajectory of the particle as determined by the BPC tracking information. The results are shown both at the EM scale as well as for the case where radial weights - both for the standard and the simplified setup - are applied. Additional point-specific (PS) weights have also been applied for comparison as summarized in Chapter 10. Topological clustering of cells is used both for the data and in extracting the weights. 


\section{Appendix B}

\section{Radial Profiles of Included Cells}

The physical volumes corresponding to the different readout channels or cells in the ATLAS Forward Calorimeters are not identical. In order to best interpret the plots in Section 8.2.2 however, at least as an initial step, it becomes useful to assume that they are. What will be shown is that under this assumption in addition to the assumption that the cells are uniformly distributed throughout the FCal modules, a linear increase in the number of cells contained within each successive radial bin is to be expected.

Figure B.1 shows the actual radial binning setup used in the FCal test beam analysis, where the active regions of the FCal are shown in grey. Here one can note the regions for which the previously mentioned assumptions are clearly not valid: the inner region corresponding to the position of the LHC beam pipe, and the outer radius of the FCal modules beyond which clearly no cells exist. What is not shown, however, are the surface areas corresponding to the individual readout channels which further invalidates the above assumptions given that, for example, the cells located nearest the central region 


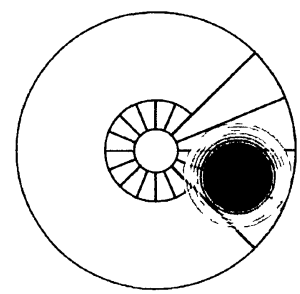

FCal1C

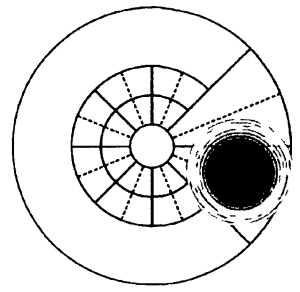

FCal2C

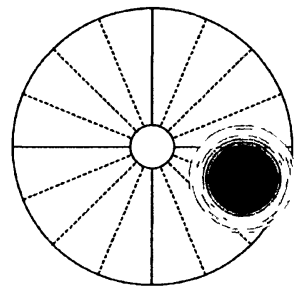

FCal3C

Figure B.1: A visual representation showing the radial binning of the cells, of which only those shown in grey were included for the 2003 Test Beam. The figure is repeated from Chapter 8 for visualization purposes.

of the FCal modules are significantly smaller than those at larger radii.

Returning to the case for which the above conditions are assumed to be true however, if the radius of the $n^{\text {th }}$ radial bin has a value of $r_{n}$, then the outer radius would have a value of $r_{n}+1$ given that each radial bin has a width of $1 \mathrm{~cm}$. The area of this bin, $A_{n}$, would then be given by:

$$
A_{n}=\pi\left[\left(r_{n}+\mathbb{1}\right)^{2}-r_{n}^{2}\right]
$$

from which it follows that:

$$
A_{n}=\pi\left(2 r_{n}+1\right)
$$

It can be seen that for cells of equal area which are distributed uniformly on a surface, a linear relationship exists between the number of cells and the radial bin number. This can be used as the basis when examining the plots in Section 8.2.2. The deviations from such a linear increase can then be reasoned based on the particular geometry in question. 


\section{References}

[1] Fermı Natıonal Accelerator Laboratory. http://www.fnal.gov/.

[2] The ATLAS Experiment at CERN. http://atlas.ch.

[3] L. Evans and P. Bryant. LHC Machıne. J. Inst., 3, Aug. 2008. S08001.

[4] G. Aad et al. The ATLAS Experiment at the CERN Large Hadron Colluder. J. Inst., 3, Aug. 2008. S08003.

[5] C. Amsler et al. Physics Letters B: Review of Particle Physics. Elsevier, 667 edition, July, 2008.

[6] R. Wigmans. Calorimetry: Energy Measurement in Particle Physics. Oxford Science Publications, 2000.

[7] W. Heitler. The Quantum Theory of Radiation. Oxford Clarendon Press, 3rd edition, 1953.

[8] R. Fernow. Introduction to experimental particle physics. Cambridge University Press, 1986.

[9] C. Fabjan and T. Ludlam. Calorimetry in high energy physıcs. Ann. Rev. Nucl. Part. Scı., 32:335-389, 1982.

[10] A. Sessoms et al. The segmented calormeter: A study of hadron shower structure. Nuc. Inst. Meth., 161:371-82, 1979. 
[11] T.A. Gabriel et al. Nucl. Instr. and Meth., A338:336, 1994.

[12] J. Artamonov et al. The ATLAS Forward Calorimeters. J. Inst., 3, Feb. 2008. JINST 3 P02010.

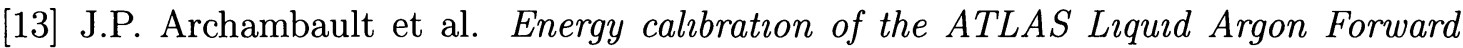
Calorimeter. J. Inst., 3, Feb. 2008. J P Archambault et al 2008 JINST 3 P02002.

[14] J.C. Armitage et al. Electron signals in the Forward Calorimeter prototype for ATLAS. J. Inst., 2, Nov. 2007. J C Armitage et al 2007 JINST 2 P11001.

[15] C. Bovet, S. Milner, and A. Placci. The CEDAR Project: Cerenkov Differentral Counters with Achromatıc Ring Focus. CERN Rev., Aug. 1975.

[16] J.P. Archambault et al. Position measurements and response uniformity of the LAr FCal Prototype for ATLAS. Proceedings of the 7th International Conference on Calorımetry in Hıgh Energy Physıcs, 1998. World Scientific.

[17] L. Heelan. Calibratıon of the ATLAS Forward Calorimeter and a Monte Carlo Study of the Top Dilepton Pair Productıon Cross-Sectıon. PhD thesis, Carleton University, Ottawa, Canada, 2010. PhD Dissertation - Pending Publication.

[18] R. Brun et al. ROOT: A data analysıs framework. http://root.cern.ch/drupal/.

[19] G. Pospelov. The overvıew of the ATLAS local hadronıc calıbratıon. J. Phys.: Conf. Series, 160, 2009. 012079, on behalf of the ATLAS hadronic calibration group.

[20] T. Barillari et al. Local Hadronıc Calıbratıon. Jan. 2009. ATL-LARG-PUB-2009-001.

[21] M. Schram. Performance of the ATLAS Forward Calorimeter and Search for the Invisıble Hıggs via Vector Boson Fusıon at ATLAS. PhD thesis, Carleton University, Ottawa, Canada, 2008. PhD Dissertation.

[22] A. Savine. U. Arizona, General weight derivation algorithm developed through correspondence within FCal Test Beam Group. 